Göttinger Zentrum

für Biodiversitätsforschung und Ökologie

- Göttingen Centre for Biodiversity and Ecology -

\title{
Species delimitation, phylogeography and population genetics of the endemic Malagasy dwarf lemurs (genus Cheirogaleus)
}

Dissertation zur Erlangung des Doktorgrades der Mathematisch-Naturwissenschaftlichen Fakultäten der Georg-August-Universität zu Göttingen

\author{
vorgelegt von \\ Dipl.-Biol. \\ Linn Fenna Groeneveld
}

aus

Göttingen

Göttingen, Februar, 2008 

Referent: Prof. Dr. Peter M. Kappeler

Korreferent: Prof. Dr. Gert Wörheide 



\section{Contents}

1 General Introduction 1

$1.1 \quad$ Madagascar and the dwarf lemurs . . . . . . . . . . . . . . . . . . . 1

1.2 Species delimitation - Who is who? . . . . . . . . . . . . . . . . . 4

1.3 Phylogeography - Species in space and time . . . . . . . . . . . . . . . 5

1.4 Population genetics - Genetic substructure within a species . . . . . . . . . 5

2 Morphometrics of the genus Cheirogaleus: what do museum and field specimens $\begin{array}{ll}\text { tell us about dwarf lemur diversity? } & 7\end{array}$

$2.1 \quad$ Introduction . . . . . . . . . . . . . . . . . . . . . . . 8

2.2 Methods . . . . . . . . . . . . . . . . . . . . . . . . . 10

2.2 .1 Sampling . . . . . . . . . . . . . . . . . . . . . . . . . . 10

$2.2 .2 \quad$ Statistical analyses $\ldots \ldots \ldots \ldots$. . . . . . . . . . . . . . 14

2.3 Results . . . . . . . . . . . . . . . . . . . . . . . . . . . . . . . . . . . . . 15

$2.3 .1 \quad$ Principal component analysis . . . . . . . . . . . . . . . . 15

2.3 .2 Cluster analysis . . . . . . . . . . . . . . . . . . . . . 16

2.3 .3 Different size, same shape? . . . . . . . . . . . . . . . . 17

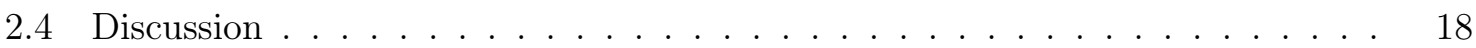

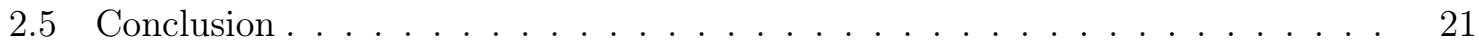

3 Species delimitation in endemic Malagasy dwarf lemurs (genus Cheirogaleus) based on mitochondrial and nuclear DNA 23

3.1 Background . . . . . . . . . . . . . . . . . . . . . . . . . . . . 24

3.2 Material and methods . . . . . . . . . . . . . . . . . . . . 26

3.2 .1 Sampling . . . . . . . . . . . . . . . . . . . . 26

3.2 .2 Laboratory work . . . . . . . . . . . . . . . . . . . . . . 32

$3.2 .3 \quad$ Phylogenetic analyses $\ldots \ldots \ldots \ldots . \ldots \ldots$

3.2 .4 Population structure $\ldots \ldots \ldots \ldots$. . . . . . . . . . . . . . . 34

3.3 Results . . . . . . . . . . . . . . . . . . . . . . . . 34

3.3 .1 Haplotype data . . . . . . . . . . . . . . . . . . . . . . 34

3.3 .2 MtDNA gene tree $\ldots \ldots \ldots \ldots \ldots$

3.3 .3 Nuclear gene trees . . . . . . . . . . . . . . . . . . . . 37

3.3 .4 Population genetic clustering $\ldots \ldots \ldots . \ldots \ldots$

3.4 Discussion . . . . . . . . . . . . . . . . . . . . . . . . . . . . 41

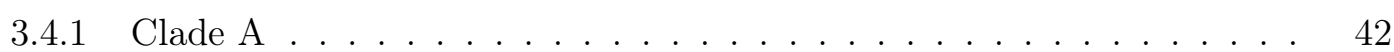


3.4 .2 Clade B . . . . . . . . . . . . . . . . . . . . . . . . 42

3.4 .3 Clade C . . . . . . . . . . . . . . . . . . . . . . . . . . . 43

3.4 .4 Comparison to Groves' taxonomy . . . . . . . . . . . . . . . . . . . . . 43

3.4 .5 Fine-scale subdivisions within the main lineages . . . . . . . . . . . . 43

3.4 .6 Geographic distributions of the species . . . . . . . . . . . . . . . . . 44

$3.4 .7 \quad$ Multifaceted approach in species delimitation in lemurs . . . . . . . . 44

3.5 Conclusion . . . . . . . . . . . . . . . . . . . . . . . . . 44

4 Dwarf lemurs in space and time: Phylogeography of the genus Cheirogaleus 46

4.1 Introduction . . . . . . . . . . . . . . . . . . . . . 47

4.2 Material and methods . . . . . . . . . . . . . . . . . . . . . . . . . . . . 49

$4.2 .1 \quad$ Concordance with biogeographic hypotheses . . . . . . . . . . . . . . 49

4.2 .2 Time divergence estimation $\ldots \ldots \ldots \ldots$. . . . . . . . . . . 51

4.3 Results . . . . . . . . . . . . . . . . . . . . . . . . . . . . . . . . . 53

4.3 .1 Concordance with 'centers-of-endemism' hypothesis . . . . . . . . . . . 53

4.3 .2 Concordance with 'biogeographic' zones based on phytogeography. . . 54

4.3 .3 Ancestral area reconstruction . . . . . . . . . . . . . . . 55

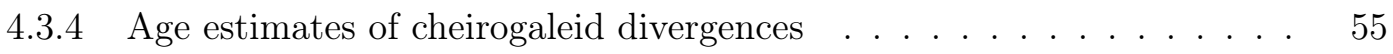

4.4 Discussion $\ldots \ldots \ldots \ldots \ldots$

$4.4 .1 \quad$ Concordance with published biogeographic hypotheses . . . . . . . . . 64

4.4 .2 Time divergence estimates . . . . . . . . . . . . . . . . 66

\begin{tabular}{|lll}
4.4 .3 & Age of cheirogaleid species in the context of biogeographic hypotheses & 68
\end{tabular}

4.5 Conclusion $\ldots \ldots \ldots \ldots$. . . . . . . . . . . . . . . . . . . . . . . . 69

5 Genetic structure, lack of sex-biased dispersal and behavioral flexibility in the pairliving fat-tailed dwarf lemur, Cheirogaleus medius $\quad 70$

5.1 Introduction . . . . . . . . . . . . . . . . . . . . . 71

5.2 Materials and methods . . . . . . . . . . . . . . . . . . . . . . 73

$5.2 .1 \quad$ Trapping and sample collection . . . . . . . . . . . . . . . . . . 73

5.2 .2 Sequencing . . . . . . . . . . . . . . . . . . . . . . . . 74

$5.2 .3 \quad$ Population genetic analyses $\ldots \ldots \ldots \ldots$. . . . . . . . . . . . 74

5.2 .4 Demographic analyses . . . . . . . . . . . . . . . . . 75

5.2 .5 Spatial analyses . . . . . . . . . . . . . . . . . 76

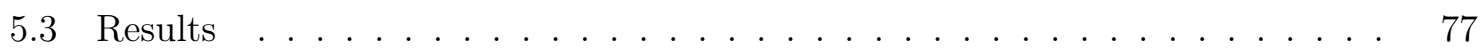

$5.3 .1 \quad$ Genetics . . . . . . . . . . . . . . . . . . . . . . 77

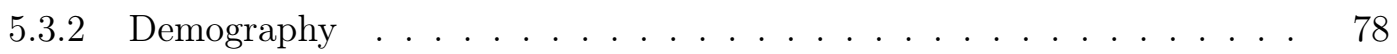

5.3 .3 Spatial patterns $\ldots \ldots \ldots \ldots$. . . . . . . . . . . . . . . . 79

$5.3 .4 \quad$ Cytochrome oxidase subunit I . . . . . . . . . . . . . . . . . 79

5.4 Discussion $\ldots \ldots \ldots \ldots \ldots \ldots$

$5.4 .1 \quad$ Genetic variability and logical prerequisites . . . . . . . . . . . . . 81

5.4 .2 Population biology $\ldots \ldots \ldots \ldots$. . . . . . . . . . . . . . . 82 
5.4 .3 Population dynamics of sympatric species . . . . . . . . . . . . . . 82

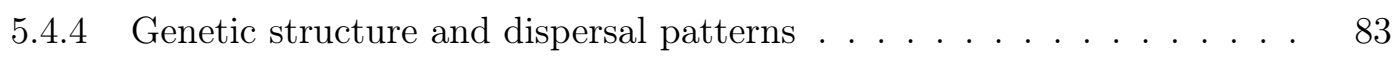

5.5 Conclusions $\ldots \ldots \ldots \ldots \ldots \ldots$

6 General Conclusion 86

$6.1 \quad$ Summary of results $\ldots \ldots \ldots \ldots$. . . . . . . . . . . . . 86

$6.2 \quad$ Main research questions regarding Madagascar's natural heritage $\ldots \ldots . . \quad 88$

6.3 Methodology . . . . . . . . . . . . . . . . . . . . . . . . . . . . . . . 91

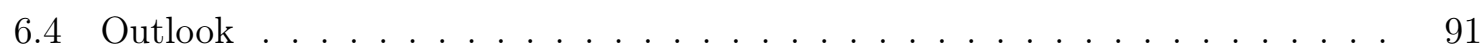

\begin{tabular}{ll}
\hline Bibliography & 93
\end{tabular}

A Appendix:

$\begin{array}{ll}\text { Species delimitation - Morphometrics } & 112\end{array}$

B Appendix:

Species delimitation - Genetics

C Appendix:

Phylogeography 



\section{General Introduction}

\subsection{Madagascar and the dwarf lemurs}

Madagascar is one of the eight hottest biodiversity hotspots of the world (Myers et al., 2000). It is home to a remarkable $11 \%$ of all primate species and subspecies, and $21 \%$ of all primate genera, all of which are endemic and most likely of a single African origin (Yoder et al., 1996). This extraordinary lemuriform fauna makes this island in the Indian Ocean a top priority for primate conservation (Mittermeier et al., 2006). Only 10\% of the islands forests are still intact and the remaining fragments are dwindling rapidly due to anthropogenic pressures (Myers et al., 2000). Since the arrival of humans on the island around 2000 years ago, many of the larger-bodied primates have become extinct (Burney et al., 2003, 2004). Based on subfossil remains we know that at least 8 genera and 16 species have become extinct in the past millennium (Godfrey et al., 1999, Mittermeier et al., 2006).

Among the smaller-bodied taxa, on the other hand, we are recording an increase of species numbers. This is mostly due to increased field work in remote areas, the incorporation of molecular methods to delineate species and a paradigm shift in what primatologists call a species (Yoder et al., 2005, Tattersall, 2007). Increased species diversity has been especially pronounced in the family Cheirogaleidae, a clade of small-bodied, arboreal and nocturnal lemurs. Most of the new species are found in the genus Microcebus, the mouse lemurs, which has septupled in species numbers in the last 25 years (e.g. Schmid and Kappeler, 1994, Zimmermann et al., 1998, Rasoloarison et al., 2000, Kappeler et al., 2005, Andriantompohavana et al., 2006, Louis et al., 2006, Olivieri et al., 2007).

Another member of the family Cheirogaleidae, the genus Cheirogaleus (dwarf lemurs), has received far less attention from the scientific community, at least in terms of its taxonomy. This genus was first described in 1812 and consisted of three species C. minor, C. medius and C. major (Geoffroy Saint-Hilaire, 1812). During the 20th century most authorities accepted a two-species taxonomy. C. medius was considered to be limited to the dry western forests, while C. major was thought to inhabit the eastern rainforests. In the year 2000 Colin Groves revised the genus and accepted a total of seven species: C. major, C. medius, C. sibreei, C. adipicaudatus, C. crossleyi, C. ravus and C. minusculus (Groves, 2000a). His revision was based entirely on morphological data from museum specimens and therefore does not contain clear information on the geographic distributions and the current existence of these species in the field.

The knowledge about general life history traits, ecology, physiology and behavior of the different dwarf lemur species is very unevenly distributed. Only two species, C. medius and 
C. major, have been the subject of detailed studies; while no data are available for the remaining species.

Most data are available for C. medius, the fat-tailed dwarf lemur (Müller and Thalmann, 2002). In addition to data gained from anecdotal observations, short term field studies or from animals kept in captivity (Petter et al., 1977, Hladik et al., 1980, Wright and Martin, 1995. Petter-Rousseaux, 1980), two longterm studies have supplied data on general aspects of the biology of the fat-tailed dwarf lemurs. One study was carried out in Kirindy forest in western Madagascar (Fietz and Ganzhorn, 1999, Fietz, 1999b a, Fietz et al., 2000, Fietz and Dausmann, 2003) and the other in northwestern Madagascar at Ampijoroa Forestry Station (Müller, 1998, 1999a b c).

Dwarf lemurs are the only primate genus that is known to undergo extended periods of torpor (=hibernation). It is assumed that this is a reaction to food shortage in the dry period of the austral winter (Petter et al., 1977, Hladik et al., 1980, Petter-Rousseaux, 1980, Wright and Martin, 1995, Fietz and Ganzhorn, 1999, Dausmann et al., 2000, 2004). The body mass of these animals therefore shows pronounced seasonal changes. Cheirogaleus medius individuals in Kirindy forest are capable of almost doubling their body mass from $124 \pm 13.5 \mathrm{~g}$ to $234 \pm$ $45.2 \mathrm{~g}$ in a few weeks before onset of hibernation for about 7 months (Fietz and Ganzhorn, 1999, Dausmann et al. 2000, 2004). The diet of $C$. medius mainly consists of fruits and flowers; supplemented with nectar, gum, insect exudates and small invertebrates (Petter et al., 1977, Hladik et al., 1980, Fietz and Ganzhorn, 1999, Lahann, 2007b). Adult pairs live in largely overlapping individual home ranges of around 1-2 ha (Kirindy: $1.6 \pm 0.5$ ha; Ampijoroa: range of 0.9 - $2.6 \mathrm{ha}$ ), often together with their presumed offspring (incl. yearlings). Their social organisation can therefore be described as pair-living (Müller, 1998, Fietz and Ganzhorn, 1999, Müller, 1999c a b). The mating system was consequently proposed to be monogamy (Fietz, 1999b). A paternity study carried out for the Kirindy forest population, however, detected a high (44\%) rate of extra-pair paternities, showing that the mating system can not be described as strictly monogamous (Fietz et al. 2000). One special trait exhibited by C. medius is paternal care (Fietz and Dausmann, 2003). Dwarf lemurs in general are highly seasonal breeders, with mating occurring directly after emergence from hibernation. Gestation length of $C$. medius is about 62 days with a litter size of generally 2-3 young (Müller and Thalmann, 2002). Cheirogaleus medius females in Kirindy, however, have been shown not to exhibit estrous synchrony (Fietz, 1999b).

Most of the data available for C. major is from a population in southeastern Madagascar at Mandena (Lahann, 2007a b). Further data are available from a study conducted in an eastern rainforest, the forêt d'Analamazoatra, near Andasibe (Ganzhorn, 1988, 1989) and from a study carried out at the Ranomafana National Park Biological Research Station in southeastern Madagascar (Wright and Martin, 1995).

Torpor in C. major was described to last from 3-6 months (Petter et al., 1977, Wright and Martin, 1995). Cheirogaleus major individuals at Mandena, just like C. medius, show changes in body mass throughout the year: $314 \pm 35.9 \mathrm{~g}$ in November and $414 \pm 46.1 \mathrm{~g}$ in February (Lahann, 2007a). Their diet is very similar to that of C. medius; at Mandena no difference 
in plant species consumed, were found between the species (Ganzhorn, 1988, 1989, Lahann, 2007b). In Ranomafana C. major is reported to feed mainly on fruits and nectar, with nectar being an important part of the diet during November and December (Wright and Martin, 1995). As in C. medius, adult pairs live in extensively overlapping home ranges of about 4 ha (Ranomafana: $\sim 4$ ha, Mandena: median 4.4 ha), together with their presumed offspring (Lahann, 2007a, Wright and Martin, 1995). Therefore their social organisation is also best described as pair-living. An overall monogamous mating system is assumed, but has not been confirmed through paternity analyses. In contrast to C. medius, C. major individuals were observed to be in close proximity of presumed family members $(<10 \mathrm{~m})$ in $49 \%$ of observations at Mandena (Lahann, 2007a). This gregarious foraging behavior has also been documented for individuals from the forêt d'Analamazoatra (Kappeler, 1997). Cheirogaleus major females in Ranomafana were observed building nests before giving birth (Wright and Martin, 1995), but this behavior was not observed at Mandena (Lahann, 2007a).

From the above outlined state of the art regarding the genus Cheirogaleus the following main questions arise and are addressed in this dissertation:

1. Is the current taxonomy, which recognizes seven species, supported by new data from the field?

a) To what conclusion regarding the number of Cheirogaleus species does the analysis of morphological data from field and museum specimens lead?

b) What can be concluded from the analyses of mitochondrial DNA and nuclear DNA markers in terms of species delimitation?

2. How are the extant Cheirogaleus species distributed geographically and how did those patterns arise?

a) Can existing biogeographic hypotheses for Madagascar be supported by new distributional data and a reassessed Cheirogaleus taxonomy?

b) When did the different Cheirogaleus species begin to differentiate and how do their time divergence estimates compare to closely related taxa?

3. What can be deduced from the population genetic structure of a $C$. medius population from western Madagascar?

a) What does the present population genetic structure of a $C$. medius population in western Madagascar reveal about the demographic history of that population?

b) Which sex disperses in C. medius and what is the consequence in terms of population genetic structure?

c) Are these data representative for the whole species?

The three main questions are addressed in detail in the following chapters. Question 1 is divided into two chapters due the complexity of species delimitation: The morphological aspect is discussed in Chapter 2, while Chapter $\mathbf{3}$ is focused on the genetic aspect of 
species delimitation. Question 2, which deals with phylogeography, is addressed in Chapter 4, while question 3, which is concerned with population genetics is discussed in Chapter 5.

In the following sections of this general introduction some theoretical background information for each of the three main questions ('Species delimitation', 'Phylogeography' and 'Population genetics') is given in order to clarify the context.

\subsection{Species delimitation - Who is who?}

Wanting to classify what is around us, animate and inanimate alike, seems to be an intrinsic human desire. What Carolus Linnaeus started off with the introduction of the binomial nomenclature has now grown into a full-fledged discipline of the biological sciences. Taxonomy, as a discipline, is often viewed as a boring and very static field, but the simple nature of the matter implies that the opposite is true. Every classification is treated as a scientific hypothesis, which if new data are collected and new evidence is found, needs to be reviewed and possibly changed accordingly (e.g. Groves 2000b). But not only the type and quantity of data available influences the evaluation of the hypotheses, also the framework, namely the species concept, within which one operates matters greatly. The species category is the biologically most significant unit in the hierarchical system. Species are the units that are used for comparisons in all other biological fields, they are the fundamental unit in biodiversity assessments and they are the unit within which biological properties can be (to a certain extent) extrapolated (e.g. Myers et al., 2000, de Queiroz, 2005). And even though most biologists would agree on those properties of a species, no one species definition/concept is accepted by all (e.g. Hey, 2001).

The problem of defining the species category is a very old one. In 1859 Darwin remarked upon the subject: "No one definition has yet satisfied all naturalists; yet every naturalist knows vaguely what he means when he speaks of a species (Darwin, 1859)." Today the most common and most widely accepted species concept is the Biological Species Concept (BSC) by Ernst Mayr. The quintessence of which is that species are "[...] groups of actually or potentially interbreeding natural populations, which are reproductively isolated from other such groups (Mayr, 1947)." For sexually reproducing sympatric species this is an operational definition, but it becomes hard to call the case when dealing with allopatric populations. Partly due to these problems in diagnosing allopatric populations many other biologists came up with their own species definitions. Depending on their specific area of work, each author stressed a certain property, that others again deemed as insignificant. An ecologist for instance would stress niche differences, a paleontologist would tend to emphasize morphological differences, while a systematist would highlight monophyly and diagnosability. This lead to quite a proliferation of different definitions/concepts; with two dozens different concepts discussed in a review by Mayden (1997).

At the end of the 20th century Kevin de Queiroz attempted to reconcile the BSC with many of the contemporary lineage-based concepts and devised the general metapopulation lineage concept de Queiroz, 1998, 2005). The species concept, in this case, is simply that 
a species is a metapopulation lineage that is evolving separately from others. This is in line with current activity in lemur taxonomy, even though this topic often is not covered explicitly. The properties that in other species concepts are necessary properties, such as diagnosability, reproductive isolation, and ecological niche differentiation, in this case become contingent properties, which can be equated with species criteria.

As already mentioned above, the current taxonomy of the genus Cheirogaleus is not corroborated by multiple lines of data and the presence of several recognized species in the field remains unconfirmed. In order to provide a robust assessment of species diversity within the genus Cheirogaleus, I propose that multiple and independent types of data should be used. Therefore morphological distinguishability and genealogical exclusivity, which was assessed with a mitochondrial and three independent nuclear markers, were chosen as species criteria in an effort to delineate dwarf lemur species. Careful examination of the data available and assessment of whether the amount of data is sufficient for certain conclusions is indispensable. These topics are covered in Chapter 2, which deals with the morphological aspect of species' delimitation, and Chapter 3, which is focused on the genetic structure of the genus.

\subsection{Phylogeography - Species in space and time}

Once lineages, or species, have been clearly defined and their present day distributions have been uncovered, the next obvious question that arises is how the patterns that we see today arose. The discipline of phylogeography, as a subdiscipline of biogeography, is concerned with this question and places special emphasis on the historical aspect of the contemporary spatial distributions (Avise, 1987, 1996, 1998, 2000). The units of interest are classically gene lineages. Usually, mtDNA variation is used to reconstruct phylogenies, which are then geographically plotted and the spatial relationships among or within the lineages are used to deduce the historical patterns and processes that shaped the contemporary distributions Avise, 1998, Hewitt, 2004). As the field expanded, other genetically based traits, such as nuclear DNA, morphological or behavioral characteristics, became units of focus (Avise, 1998). The term 'coalescent theory' is closely linked to the discipline of phylogeography and is applied to the mathematical and statistical properties of gene genealogies (e.g. Kingman, 1982, Watterson, 1984). Based upon this framework, methods have been devised that allow the estimation of past population parameters, such as the dates of historical bottlenecks, the size of ancestral populations, the location of refugial areas and the dates of divergences (Hewitt, 2004). In Chapter 4 I explore the historical past in space and time of the genus Cheirogaleus, including a comparison to the closely related genus Microcebus.

\subsection{Population genetics - Genetic substructure within a species}

"Evolution is the process of change in the genetic makeup of populations" Graur and Li, 2000 ). The change of gene frequencies in populations with time are the basic component of evolutionary processes. Since evolution happens at the population level, this is the next level 
of interest to someone studying the evolution of species. The genetic variability found within a population can be important baseline data for calibrating markers used on a higher level and for assessment of the viability of a population. For instance, population genetic methods can give robust estimates of $\mathrm{N}_{\mathrm{e}}$ that due socioecological effects may differ considerably from adult census data. In primates, tremendous variation in reproductive success among individuals, families and/or between the sexes can reduce the adult gamete pool sampled at each generation. Thus, in order to provide a robust assessment of the viability of a population data on socioecological effects have to be available and taken into account (Melnick and Hoelzer, 1993 . Pope, 1996). A population level study, which uses genetic data to characterize historical and present demography and relates these variables to the social system of the species, is presented in Chapter 5. 


\title{
2 Morphometrics of the genus Cheirogaleus: what do museum and field specimens tell us about dwarf lemur diversity?
}

\author{
Linn F. Groeneveld ${ }^{1} \&$ Rodin Rasoloarison ${ }^{2} \&$ Peter M. Kappeler ${ }^{1,3}$ \\ ${ }^{1}$ Department of Behavioral Ecology and Sociobiology, German Primate Center, Göttingen, \\ Germany \\ ${ }^{2}$ Départment de Biologie Animale, Université d'Antananarivo, Antananarivo, Madagascar \\ ${ }^{3}$ Institute of Zoology und Anthropology, University of Göttingen, Göttingen, Germany
}

submitted to American Journal of Physical Anthropology 


\begin{abstract}
Madagascar's evolutionary history is not well understood. Detailed knowledge of contemporary species identity and species distributions is essential for unraveling the mechanisms that shaped the endemic radiations on this island. Furthermore, it is indispensable baseline data for conservation efforts. Lemur taxonomy has experienced a drastic revision in the last decades, with species numbers skyrocketing. In the small-bodied, nocturnal mouse lemurs (genus Microcebus) the increase has been especially drastic. Species numbers in the mouse lemurs septupled within the last 25 years due to intensified field work, incorporation of molecular data and a paradigm shift in the definition of a species. The closely related dwarf lemurs (genus Cheirogaleus), which share similar habitats with mouse lemurs, have received less attention in terms of their taxonomy. The last revision of the genus was based on morphological data from museum specimens and accepted seven species: C. medius, C. major, C. crossleyi, C. adipicaudatus, C. sibreei, C. ravus and C. minusculus. The goal of this study was to verify the currently accepted taxonomy of the genus, by examining 6 external and 32 cranio-dental characters of 120 museum specimens and 36 individuals from the field. This study revealed lower diversity and a lower number of distinct morphs of dwarf lemurs than expected. We conclude that in our sample there are three distinct morphs in the genus Cheirogaleus that correspond to C. medius, C. major and $C$. crossleyi. Further sources of corroborative data are required for robust species delimitations.
\end{abstract}

\title{
2.1 Introduction
}

Little is known about the underlying mechanisms that have shaped the endemic radiations and contemporary species distributions in Madagascar (Krause et al., 1997, Wilmé et al., 2006). A detailed understanding of species identity and species distributions is essential for unraveling the evolutionary history of this island. Also, for conservation, baseline data about species identity and distributions are vital. In a country that, due to its high endemism rates and low percentages of remaining primary vegetation, belongs to the hottest biodiversity hotspots of the globe, effective conservation measures are a top priority (Myers et al., 2000). Lemurs are important flagship species due to their charismatic characteristics and highly endangered status (Mittermeier et al., 1992, Smith et al., 1997, Durbin, 1999). Nonetheless, lemur taxonomy is far from stable and new species are being described at a high rate (Isaac et al., 2004, Yoder et al., 2005, Tattersall, 2007). Drastic increases in species numbers are documented in the nocturnal sportive lemurs (Lepilemuridae) (Andriaholinirina et al., 2006, Craul et al. 2007) and the nocturnal and very small-bodied mouse lemurs (Cheirogaleidae: Microcebus) (Schmid and Kappeler, 1994, Zimmermann et al., 1998, Rasoloarison et al., 2000, Kappeler et al., 2005, Louis et al., 2006, Andriantompohavana et al., 2006, Olivieri et al., 2007). Concern has been raised about the validity of some of these newly described species (Tattersall, 2007) and an integrative approach to species delimitation incorporating multiple data sources is being advocated by many (Dunn, 2003, Lipscomb et al., 2003, Mallet and Willmott, 2003). One of the oldest and also most intuitive data sources is morphometric variability. Although cryptic species may exist within morphologically homogeneous groups, distinct morphological groups are a good first indicator of differentiation between groups. Further sources of data are subsequently needed to verify and justify a species-level delimitation.

This approach yielded new insights into mouse lemur diversity. Rasoloarison et al. (2000) found high levels of morphological variability in a study of 12 western mouse lemur popula- 
tions, upon which they based the description of three new species and a resurrection from synonymy. These species were subsequently confirmed by mtDNA analyses (Yoder et al., 2000). Previously, from the late 1970s until the1990s, the genus Microcebus was thought to comprise two species, with a grayish form found in the western dry forests and a rufus colored form in the eastern rainforests (Petter et al., 1977, Tattersall, 1982). The same two-species taxonomy, with a western grey form and an eastern rufus colored form, was accepted for the genus Cheirogaleus from 1931 until the turn of the century (Petter et al., 1977, Tattersall, 1982, Groves, 2000a). This leads to the question of whether the dwarf lemurs, being closely related to mouse lemurs, show the same patterns of diversity. The taxonomic history of the genus Cheirogaleus was more complex than outlined above, and there were several indications before 2000 that there are actually more than two extant morphs.

The genus Cheirogaleus was first described by Etienne Geoffroy St. Hilaire according to a drawing of three animals by Philibert Commerçon sent from Madagascar in 1812. These animals were postulated to be three separate species and described as C. minor, C. medius and C. major (Geoffroy Saint-Hilaire, 1812). From 1812 to 1931 the taxonomy of the genus was rather turbulent. At least 11 synonymous genus names were attributed to the genus: Cheirogaleus (Geoffroy Saint-Hilaire, 1812), Chirogaleus (Oken, 1816), Myspithecus (Cuvier, 1842), Mioxicebus (Lesson, 1840), Cebugale (Lesson, 1840), Chirogale (Gloger, 1842), Myoxicebus (Agassiz, 1845), Myoxocebus (Agassiz, 1846), Opolemur (Gray, 1872), Altililemur (Elliot, 1913), Altilemur (Weber, 1928). In 1931 the minimalist/lumper Ernst Schwarz revised the genus and accepted only two species, each comprised of two subspecies: Cheirogaleus major major, C. major crossleyi and C. medius medius, C. medius samati (Schwarz, 1931). This taxonomy remained valid for over 40 years.

In 1977 it was slightly modified by Petter et al., who kept the two C. major subspecies, but collapsed the two C. medius subspecies into just one. They believed that at least three forms of $C$. major existed, one of which remained unnamed and also speculated about further undiscovered forms in isolated regions of Madagascar (Petter et al., 1977). Thalmann and Rakotoarison (1994) reported an unusual morph in western Madagascar, where only C. medius was thought to exist; they provisionally classified this morph as C. major. Further reports of the presence of C. major in central western Madagascar (Bongolava and Bemaraha areas) were published in the following years (Ausilio and Raveloanrinoro, 1998, Thalmann, 2000).

No new species had been described since 1896 (Forsyth Major, 1896) until in 2000 Colin Groves revised the genus based on re-analysis of the available museum material. He accepted a total of seven species: keeping C. major and C. medius, resurrecting C. sibreei and C. adipicaudatus from synonymy, elevating $C$. crossleyi to full specific status and newly describing C. ravus and C. minusculus (Groves, 2000a). The geographical distribution of these species is not known in full detail due to the limited number of museum specimens available and their poor record of provenance. In 2005 Hapke et al. (2005) reported three distinct morphs in the forest fragments of the Fort Dauphin region (southeast). Employing morphological and genetic analyses, they concluded that the third unknown morph (besides the expected $C$. medius and C. major) was C. crossleyi, thus considerably extending the distribution of this 
species, which was previously thought to be restricted to the northeast. In a recent survey, Rasolofoson et al. (2007) described the presence of four sympatric Cheirogaleus species in the region of Makira, a rain forest area in northeastern Madagascar.

The aim of this study was to elucidate the diversity of dwarf lemurs based on morphological data obtained from new field specimens. We hope to verify Groves' taxonomy and add to the knowledge of geographical distributions of the species described by Groves. Since the last revision of the genus was based solely on museum specimens, we place special emphasis on adding new data from the field. Furthermore, we aim to clarify the species identity of unlabeled museum specimens. We therefore examined the collections of five European museums and caught 44 individuals during 14 expeditions in the field.

\subsection{Methods}

\subsubsection{Sampling}

Field samples from a total of 44 individuals of dwarf lemurs were collected from March 2003 to May 2007 at 14 sites in Madagascar (Collection sites and other localities mentioned in the text are shown in Fig 2.1). The majority of specimens used in this project were prepared as standard museum skins with associated skulls and postcranial skeletons. A maximum of three individuals per site, amounting to 32 individuals, were sacrificed, using a dart gun, and preserved as morphotypes. Tissue samples, ectoparasites, and stomach contents were also saved in $70 \%$ ethanol. Additionally, animals were caught using Sherman live traps. One hundred traps were set along two or three transects for an average of 11 nights per site and baited with pieces of banana. A total of 12 individuals were trapped and released at the site of capture on the following day at dusk. Tissue for molecular analyses of these individuals was obtained by ear clipping during anesthesia with GM2 (Rensing, 1999). Only adult specimens are included in the analyses below, amounting to 36 individuals from the field. Furthermore, a total of 120 adult specimens housed at the natural history museums in Paris (MNHN, 60 individuals), London (NHM, 30 individuals), Leiden (Naturalis, 18 individuals), Frankfurt (Senckenberg, 2 individuals) and Berlin (ZMB, 10 individuals) were included (see Appendix: Table A.1). Six standard external morphometric measurements (see below) were taken from all field individuals, whereas 32 cranio-dental measurements were taken from specimens preserved as morphotypes, as well as from the museum specimens. All measurements were taken only by R. Rasoloarison to exclude inter-observer error.

\section{External measurements}

The following external measurements were taken from live specimens, as well as from the morphotypes before the onset of rigor mortis.

- Ear length (EAR): from the notch at the base of the to the distalmost edge of the pinna. 


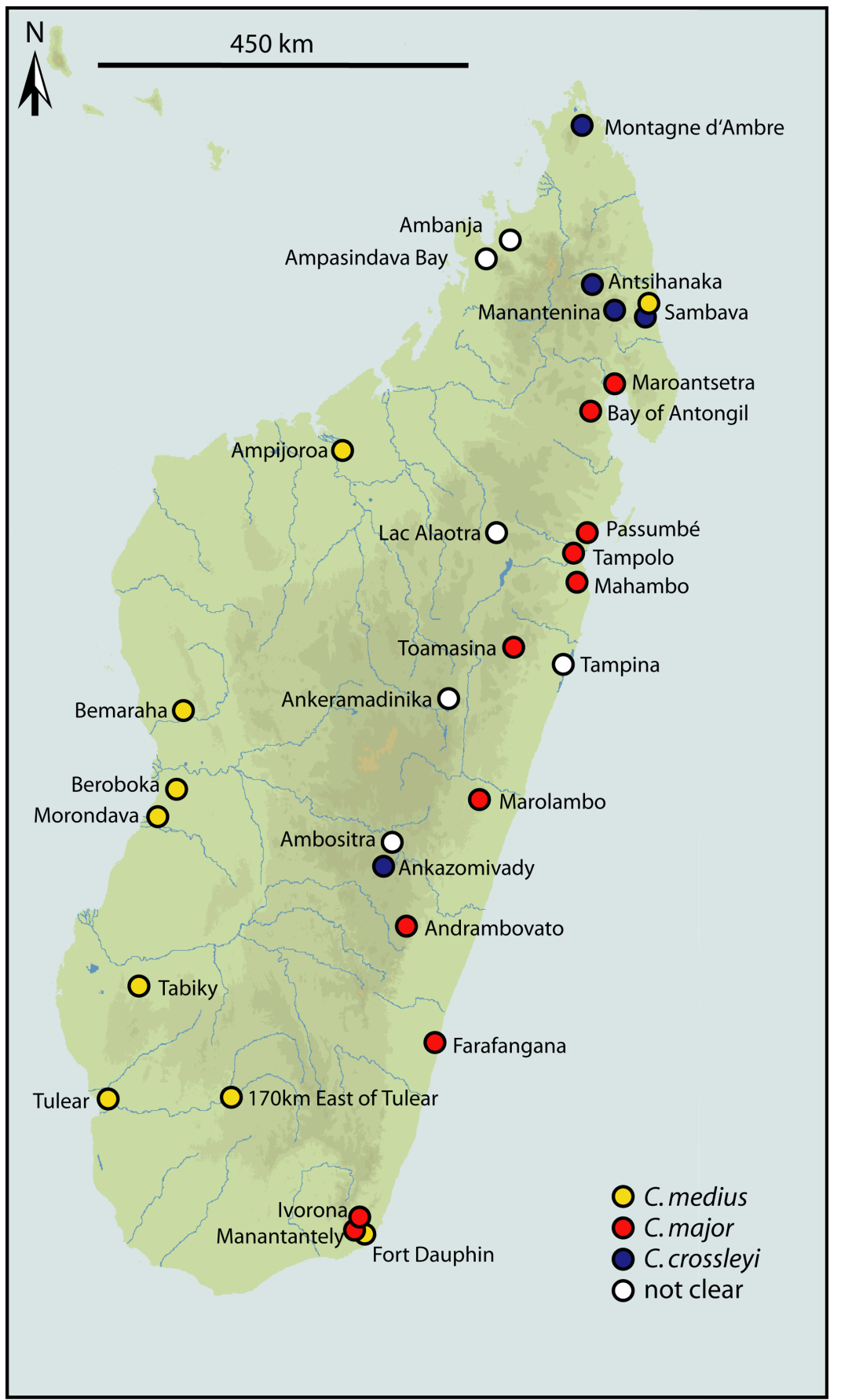

Figure 2.1: Sampling localities. Sampling sites of both field samples and museum specimens included in the analyses of this study are marked with circles. Colors indicate that individuals found at the respective site were classified as C. medius (yellow), C. major (red) and C. crossleyi (blue), or could not be clearly classified (white) by the analyses presented in this study. The Sambava locality is marked by two circles, since two different species were collected at this site. 
- Head and body length (HB): from the tip of the nose to the distalmost point of the body (at base of tail).

- Hindfoot length $(\mathbf{H F})$ : from the back edge of the heel to the tip of the longest toe (not including claw).

- Tail length (TAIL): from the base of the tail (at right angles to the body) to the end of the distalmost vertebra, excluding terminal hair tufts.

- Total length (TL): from the tip of the nose to the end of the caudal vertebra, excluding terminal hair tuft.

- Mass (W) measured with Pesola spring scales to $\pm 0.5 \mathrm{~g}$ for individuals $<100 \mathrm{~g}$ and to $\pm 1 \mathrm{~g}$ for those $>100 \mathrm{~g}$.

\section{Cranial and dental measurements}

Cranial measurements were obtained via digital calipers to an accuracy of $\pm 0.1 \mathrm{~mm}$. The following dental notations are used: premaxillary and maxillary teeth are denoted by upper case and mandibular teeth by lower case. The following abbreviations are used: incisor $(\mathrm{I} / \mathrm{i})$, canine $(\mathrm{C} / \mathrm{c})$, premolar $(\mathrm{P} / \mathrm{p})$, molar $(\mathrm{M} / \mathrm{m})$.

- Basal skull length (BASL): from the anterior edge of the premaxillae to the anteriormost point on the lower border of the foramen magnum.

- Condylobasal axis (M12): distance between the basal mandibular plane to the condylar projection.

- Condylobasal length (COBL): from the anterior edge of premaxillae to the posteriormost projection of occipital condyles.

- Coronoidbasal axis (M13): distance between the basal mandibular plane to the coronoid process.

- Frontal length $(\mathbf{F r L})$ : greatest length from fusion with the nasal bone to the fusion with the parietal bone.

- Greatest orbital diameter (OrDiL): greatest diameter of the orbit from the malar fossa.

- Greatest skull length (GSKL): from the rostrum to the back of braincase.

- Least orbital breadth (DiOr): smallest distance dorsally between the orbits.

- Lower postcanine tooth row (MR): distance between p3 and m3, at level of cusp.

- Mandibular length (ML): greatest length of the mandible from the anteriormost point of the symphysis to the condyle.

- Nasal length (NasL): greatest length of nasal bone (rostral end to fusion with frontal). 
- Nasal width (NasW): greatest width across nasal bone anteriormost.

- Occipital length (OccL): greatest dimension following the nasal-frontalparietal line.

- Occipital width $(\mathbf{O c c W})$ : greatest dimension of the occipital bone, perpendicular to skull length.

- Orbital transverse diameter (OrDiW): diameter of the orbit from lachrymal bone to level of zygomatic bridge fusion.

- Palatal length (PALL): from anterior edge of premaxillae to anteriormost point on posterior edge of the palate.

- Palatal width (PALW): between alveoli of second upper molars.

- Parietal length (ParL): greatest length from the fusion with the frontal to occipital bones.

- Parietal width (ParW): greatest width of the parietal bone at the fusion of the temporal line with the occipital complex.

- Skull height (SKH): from bullae to parietal bone, perpendicular to skull length.

- Greatest width of temporal fossa (TeFoL): from the posterior most point of the zygomatiotemporal suture to the posteriormost point of the maxilla posterior to M3.

- Temporal line (TpLi): distance between parietal line and temporal line, perpendicular to the former at its origin.

- Zygomatic breadth (BZ): greatest breadth across the zygomatic process perpendicular to skull length at the junction of the zygomaticoorbital suture.

- C: height of the canine from the mesial edge of the alveolus to the distalmost point of the crown.

- I1 : height of upper first incisor from the anterior border of alveolus to the distalmost point of the crown.

- I2: height of upper second incisor from the anterior border of alveolus to the distalmost point of the crown.

- M1: length of the upper first molar at greatest width of the cusp.

- M2: length of the upper second molar at greatest width of the cusp.

- M3: length of the upper third molar at greatest width of the cusp.

- P2: height of the upper second premolar from the mesial edge of alveolus to the distalmost point of the crown. 
- P3: height of the upper third premolar from the mesial edge of alveolus to the distalmost point of the crown.

- P4: height of the upper fourth premolar from the mesial edge of alveolus to the distalmost point of the crown.

\subsubsection{Statistical analyses}

Because many of the museum specimens were not completely intact, the data matrix contained missing data values. Measurements were taken from a total of 156 individuals. External measurements were only available for 36 of the 156 individuals. Cranio-dental measurements were available from 149 individuals, but of those only 78 individuals had no missing data values. The amount of missing data per individual for the remaining 71 individuals varied from $3 \%$ to $59 \%$.

As previously demonstrated for lemurs, including the genus Cheirogaleus (Kappeler, 1990. 1996), we also did not find any sex differences in any morphological variables and therefore pooled the data for males and females.

Due to the missing data we analyzed three separate data sets. The first data set ('all cranio-dental') included all cranio-dental characters ( $\mathrm{N}=78$ individuals). In the second data set ('max. individuals') we tried to maximize the number of individuals (i.e. those without missing data values) and chose only the characters PALL, PALW, NasL, NasW, C, I1, I2, M1, M2, M3, P2, P3, P4, M12, M13 and MR. This data set included 110 individuals. The third data set ('external') included only external measurements, excluding mass since this varies greatly with season (Fietz and Ganzhorn, 1999, Lahann, 2007a), and consequently only included the field samples (36 individuals).

A principal component analysis (PCA) on the correlations matrix was carried out as implemented in JMP v6.0. A plot of principal component 1 (PC1) against principal component 2 (PC2) was used to visually assess the number of distinctive clusters. Subsequently, we conducted hierarchical cluster analyses as implemented in JMP v6.0. Ward's minimum variance method was used for defining distances between clusters. The data were standardized by the mean and standard deviation of each variable (character).

To evaluate congruence between Groves taxonomy and our results, differences between species, as classified by Groves, for the first two principal components (PC) were assessed using the non-parametric Kruskal-Wallis test followed by Dunn's multiple comparisons test as implemented in GraphPAD Prism v4.0c (GraphPad Software, San Diego California USA). For these comparisons among pre-determined groups, only museum specimens assessed by Groves and individuals from the field, classified according to Groves (2000a), were included. Whenever species names are mentioned in the results, we are referring to Groves' (2000) classification.

In morphometrics, PC1 is often found to be a multivariate measure of overall size (Jolicoeur and Mosimann, 1960, Blackith and Reyment, 1971). Since C. medius and C major, as classified by Groves and confirmed by our analyses, only differed in PC1, we investigated 
whether these species are different only in size but not in shape (i.e. are 'isometric versions' of one another). We generated size-adjusted data by dividing each variable by the geometric mean of all variables for that specimen (Darroch and Mosimann, 1985, Jungers et al., 1995). Subsequently, the pairwise average taxonomic distances among all included specimens were calculated. A zero average taxonomic distance between any two specimens is indicative of same shape (Sneath and Sokal, 1973, Jungers et al., 1995).

We consider error probabilities of $<5 \%$ to be statistically significant.

\subsection{Results}

\subsubsection{Principal component analysis}

For all three data sets principal component analysis, according to the latent root criterion and scree plots, identified two informative components. For the 'all cranio-dental' data set the first two components explained $83.60 \%$, for the 'max. individual' data set $86.51 \%$ and for the 'external' data set $94.68 \%$ of the total variance in the respective sample (see Table 2.1).

Table 2.1: Principal component analysis for three data sets: 'all cranio-dental', 'max. individuals' and 'external'. Two components were retained and interpreted. The eigenvalue, percentage of variance explained by each component and the cumulative percentage of explained variance is given for each of the three data sets.

\begin{tabular}{lcccc}
\hline & Component & Eigenvalue & \% of variance & Cumulative \% \\
\hline All cranio-dental & 1 & 24.51 & 76.59 & 76.59 \\
& 2 & 2.24 & 7.01 & 83.60 \\
Max. individuals & 1 & 12.58 & 78.62 & 78.62 \\
& 2 & 1.26 & 7.89 & 86.51 \\
External & 1 & 4.17 & 83.31 & 83.31 \\
& 2 & 0.57 & 11.37 & 94.68 \\
\hline
\end{tabular}

Since there is no biologically meaningful difference between the 'all cranio-dental' and 'max. individual' data set, we will only present the data of the max. individual data set in detail. The principal component loadings and the loadings for a varimax rotation, which incorporates an orthogonal rotation to maximize loadings on the variables, are given in Table 2.2. The interpretation of the principal components is not greatly enhanced by the rotation. All variables load heavily on the first component. Only the variables C (canine height), P2 (upper second premolar) and P3 (upper third premolar) load significantly on the second component. The subsequent analyses were therefore done directly on the principal component scores and not on the rotated factors. When plotting the first against the second PC, three clouds can be distinguished (Fig. 2.2). These roughly correspond to C. medius, C. major and C. crossleyi as classified by Groves. When testing the differences between pre-determined species for the first two PCs, there was an overall difference for the first PC and 4 pairwise differences (Kruskal-Wallis, test statistic $=58.74,7$ groups, $\mathrm{p}<0.0001$; Dunn's post test, $C$. adipicaudatus vs. C. major: $\mathrm{p}<0.01$, C. crossleyi vs. C. medius: $\mathrm{p}<0.05$, C. major vs. C. medius: $\mathrm{p}<0.001$, 


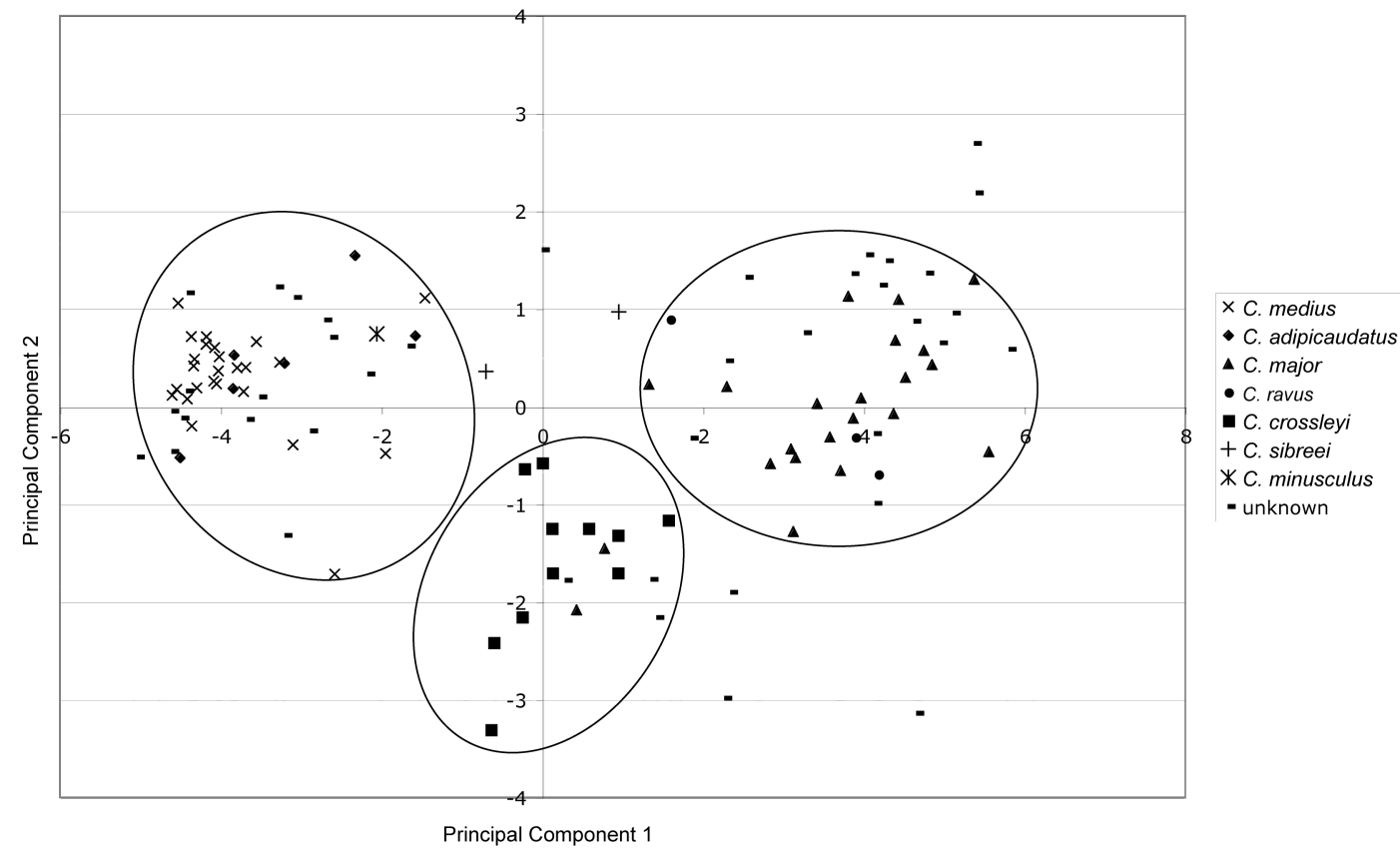

Figure 2.2: Morphometric variation among Cheirogaleus taxa. Plot of the first against the second principal component based on based on the variables PALL, PALW, NasL, NasW, C, I1, I2, M1, M2, M3, P2, P3, P4, M12, M13 and MR of 110 individuals: $\mathrm{n}_{\text {major }}=22, \mathrm{n}_{\text {ravus }}=3, \mathrm{n}_{\text {medius }}=25, \mathrm{n}_{\text {adipicaudatus }}=6, \mathrm{n}_{\text {crossleyi }}=11, \mathrm{n}_{\text {minusculus }}=1$, $\mathrm{n}_{\text {sibreei }}=2, \mathrm{n}_{\text {unknown }}=43$. Three clouds can be identified and are marked by circles.

C. medius vs. C. ravus: $\mathrm{p}<0.05$; Fig. 2.3 A). There was also an overall difference between species for the second PC (Kruskal-Wallis, test statistic $=28.18,7$ groups, $\mathrm{p}<0.0001$ ). Pairwise post-hoc comparisons revealed three pairwise differences for the second PC (Dunn's post test, C. crossleyi vs. C. adipicaudatus: $\mathrm{p}<0.01$, C. crossleyi vs. C. major: $\mathrm{p}<0.05$, C. crossleyi vs. C. medius: $\mathrm{p}<0.001$, Fig. $2.3 \mathrm{~B})$.

\subsubsection{Cluster analysis}

The result of the cluster analysis on the data set with maximized number of individuals $(\mathrm{n}=110)$ is presented in Fig. 2.4. No clear structure clustering the individuals of each of the seven species, as classified by Groves, can be found. As suggested by the PCA, assuming three clusters is most consistent with the species labels. In this case, the first cluster consists of C. medius and C. adipicaudatus individuals, plus one C. sibreei and the C. minusculus individual. The second cluster consists of $C$. crossleyi and two C. major individuals, while the third cluster is comprised of C. major and C. ravus individuals plus one C. crossleyi and one $C$. sibreei individual. The $C$. adipicaudatus and $C$. ravus individuals do not form exclusive groups within the C. medius and C. major clusters, respectively. This data set ('max. individuals') differs from the all 'cranio-dental' data set only in the placement of 4 individuals as denoted by asterisks in Fig. 2.4. Three unlabeled specimens, which for the 'max. individuals' data set are placed in the clade containing mostly C. major specimens, are placed in the clade mostly consisting of $C$. crossleyi individuals in the 'all cranio-dental' data 
Table 2.2: Principal component loadings for principal component one (PC1) and two (PC2) for both rotated and unrotated factor patter, based on 'max. individuals' data set.

\begin{tabular}{lllll}
\hline Variable & \multicolumn{2}{l}{ Unrotated factor pattern } & \multicolumn{2}{l}{ Rotated factor pattern } \\
& PC1 & PC2 & PC1 & PC2 \\
\hline PALL & 0.97 & -0.16 & 0.92 & 0.34 \\
PALW & 0.95 & -0.15 & 0.90 & 0.34 \\
NasL & 0.94 & -0.14 & 0.88 & 0.34 \\
NasW & 0.87 & -0.03 & 0.78 & 0.40 \\
I1 & 0.83 & 0.12 & 0.66 & 0.52 \\
I2 & 0.82 & -0.14 & 0.78 & 0.29 \\
C & 0.56 & 0.75 & 0.12 & 0.92 \\
P2 & 0.76 & 0.49 & 0.42 & 0.80 \\
P3 & 0.84 & 0.40 & 0.53 & 0.76 \\
P4 & 0.90 & 0.23 & 0.66 & 0.65 \\
M1 & 0.90 & -0.12 & 0.84 & 0.34 \\
M2 & 0.97 & -0.10 & 0.89 & 0.40 \\
M3 & 0.92 & -0.22 & 0.90 & 0.26 \\
ML & 0.96 & -0.13 & 0.89 & 0.36 \\
M12 & 0.95 & -0.19 & 0.91 & 0.31 \\
MR & 0.97 & -0.17 & 0.93 & 0.33 \\
\hline
\end{tabular}

set. The C. sibreei individual found in the clade comprising mostly C. major individuals for the 'max. individuals' data set, is placed in the clade containing mostly $C$. medius individuals in the 'all cranio-dental' data set.

The external data set only included individuals representing three species (C. major, $C$. crossleyi and C. medius) plus one unclassified individual. When assuming three clusters all C. medius plus the unlabeled individual form an exclusive cluster $(\mathrm{n}=5)$; all $C$. crossleyi plus three $C$. major individuals form a cluster and the remaining $C$. major individuals make up the third cluster.

\subsubsection{Different size, same shape?}

Mean intra-specific average taxonomic distances, ranging from 0.018 for C. crossleyi individuals to 0.025 for $C$. major individuals, were slightly lower than mean inter-specific average taxonomic distances (Table 2.3). These ranged from 0.028 between C. crossleyi and C. major individuals to 0.033 between $C$. crossleyi and C. medius. No average taxonomic distances of zero, indicating same shape, could be detected between taxa. Mean average taxonomic distance of 0.031 between $C$. major and C. medius does not indicate that these species differ only in size. 
A)
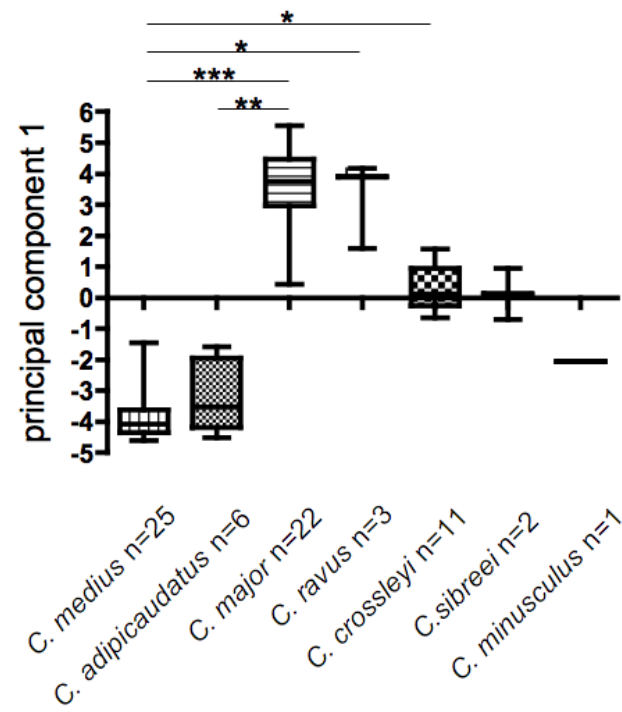

B)

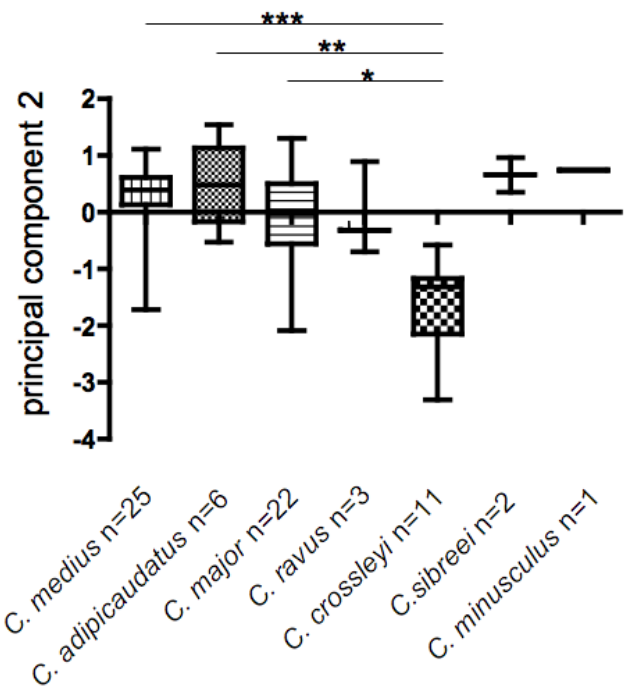

Figure 2.3: Size and shape variation. Box and whisker plots of PC1 (A) and PC2 (B) scores for each of 7 species: C. medius, C. major, C. ravus, C. crossleyi, C. sibreei, C. minusculus. Pairwise differences are denoted by asterisks. The range is indicated by the whiskers, the interquartile range is denoted by the boxes and the median value bisects the box. C. medius and C. crossleyi can be distinguished by PC1 and $\mathrm{PC} 2$, while $C$. medius and C. major only differ in $\mathrm{PC} 1$ and $C$. major and $C$. crossleyi differ in the PC2. Furthermore, pairwise differences were detected for $C$. medius vs. C. ravus and C. adipicaudatus vs. C. major for PC1 and C. crossleyi vs. C. adipicaudatus for $\mathrm{PC} 2$.

\subsection{Discussion}

Both principal component and hierarchical cluster analyses supported the existence of three distinct clusters within the genus Cheirogaleus. Both types of analyses agreed on the grouping of $C$. medius with $C$. adipicaudatus and the only C. minusculus individual and the grouping of $C$. major with C. ravus. Neither analysis showed a distinct substructure within these clusters according to species labels. One C. crossleyi individual (ZD.1948.160) was located in the C. major cluster in the cluster analysis, but was found within the C. crossleyi cloud in the PCA plot, albeit close to the C. major cloud. We therefore concluded that the cluster analysis failed to cluster this individual correctly. The two C. sibreei individuals did not fall into any of the three clouds in the PCA plot, but were located in the space between the C. medius and C. major clade. In the cluster analysis their status was ambiguous: one of the individuals (1887:66b) grouped with the C. medius individuals in the 'max. individuals' data set. The other individual (ZD.1897.9.1.160) grouped with the C. major individuals in the 'max. individual' data set, but with the C. medius individuals in the 'all cranio-dental' data set. The two individuals RMR193 and RMR194 were classified as C. major in the field 


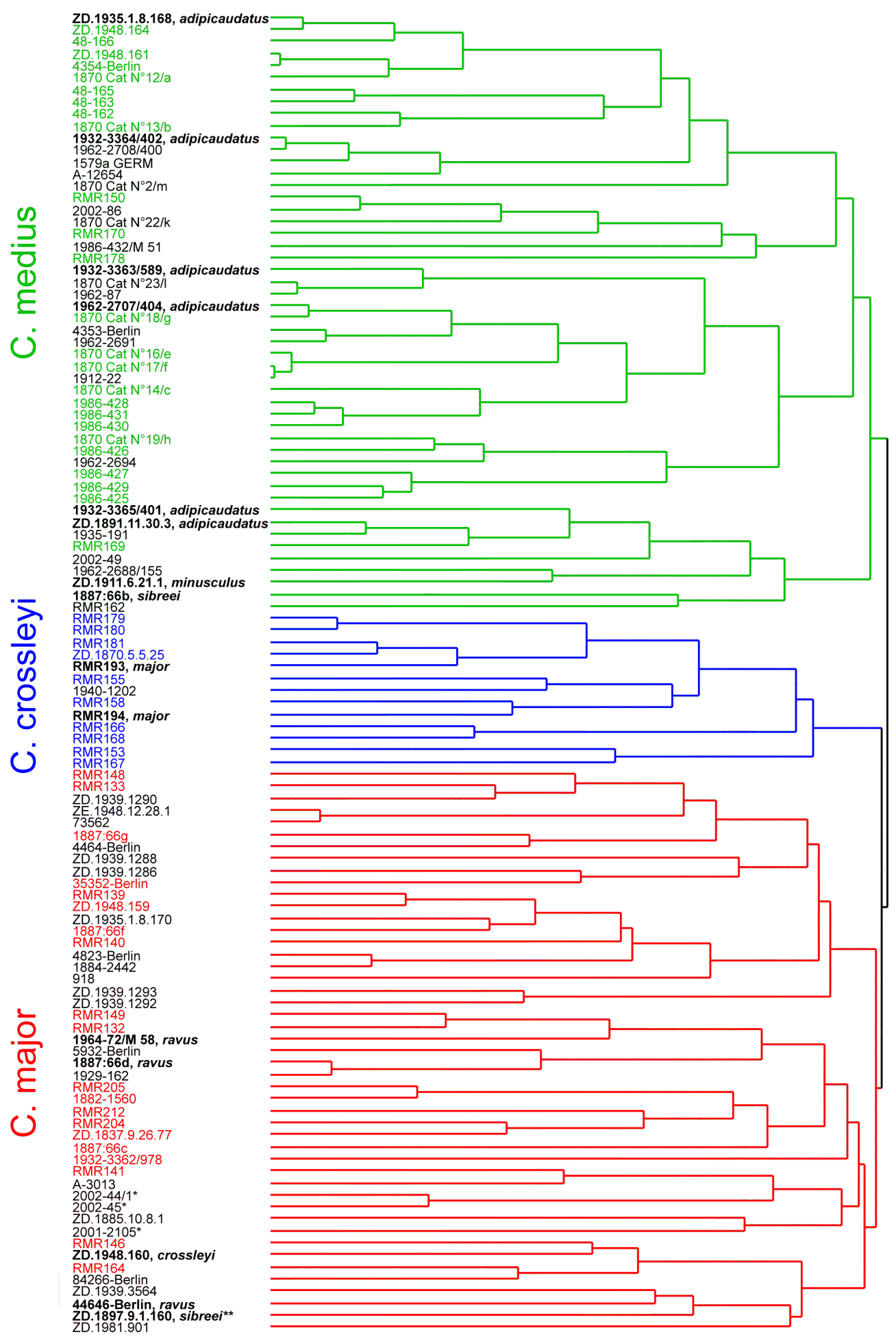

Figure 2.4: Hierarchical cluster analysis of morphometric data of Cheirogaleus taxa. Based on the 'max. individuals' data set which includes 110 individuals. The number of clusters was defined post-hoc. The first cluster includes mainly C. medius individuals (green), with C. adipicaudatus individuals interspersed. Also, the only C. minusculus and one C. sibreei individual falls into this cluster. The second cluster is composed of $C$. crossleyi individuals (blue) together with two C. major individuals. The third cluster consists of C. major individuals (red) interspersed with $C$. ravus individuals plus one $C$. crossleyi individual. ** individual found in the cluster mainly consisting of $C$. medius individuals in the 'all cranio-dental' data set. ${ }^{*}$ individual found in the cluster mainly consisting of $C$. crossleyi individuals in the 'all cranio-dental' data set. 
Table 2.3: Mean intra- and interspecific average taxonomic distances (ATD) and standard deviation $(\hat{\sigma})$ for C. medius $(\mathrm{n}=20)$, C. major $(\mathrm{n}=16)$ and $C$. crossleyi $(\mathrm{n}=9)$, as classified by Groves and confirmed by this study. Based on 32 characters and a total of 45 individuals.

\begin{tabular}{lcccccc}
\hline & \multicolumn{2}{c}{ C. major } & \multicolumn{2}{c}{ C. medius } & \multicolumn{2}{c}{ C. crossleyi } \\
& ATD & $\hat{\sigma}$ & ATD & $\hat{\sigma}$ & ATD & $\hat{\sigma}$ \\
\hline C. major & 0.025 & 0.008 & & & & \\
C. medius & 0.031 & 0.006 & 0.023 & 0.007 & & \\
C. crossleyi & 0.028 & 0.008 & 0.033 & 0.005 & 0.018 & 0.005 \\
\hline
\end{tabular}

according to Groves' taxonomy (Groves, 2000a). Due to both analyses presented here, these two individuals had to be reclassified as C. crossleyi, demonstrating how difficult it is to distinguish these two taxa in the field.

Cheirogaleus crossleyi differed from C. medius and C. major in $\mathrm{PC} 2$, which according to the loadings represented dental characteristics: canine, upper second and third premolar height. Furthermore, C. crossleyi differed from C. medius in PC1, which is often found to be an overall measure of size. Cheirogaleus medius and C. major were only separated by PC1, which suggested that C. medius differs primarily in size from C. major. The calculated average taxonomic distances, however, suggested that $C$. major and $C$. medius demonstrated scale-related changes in shape. The two species differ primarily in size, but nonetheless also in shape; they are not isometric versions of another.

We plotted the sampling localities of all individuals, which we included in our analyses on the map in Fig. 2.1. Furthermore, the sampling localities are color-coded according to species, as classified in this study (Appendix: Table A.1). Cheirogaleus medius specimens were found from the southeastern tip (22; Fort Dauphin region) throughout the Southwest $(23,24,25)$ along the west coast $(26,27,28)$ up north to Ampijoroa (29). There were two additional unexpected sampling sites: C. medius was found in Sambava (5) in the northeast and in the central highlands at Ambositra (16). Although C. medius has been reported to be present in the northeast at Daraina (Mittermeier et al., 1994, 2006), the Sambava sampling site extends the distributional range on the eastern coast by more than $100 \mathrm{~km}$ towards the South. The sampling site on the central plateau represents the C. minusculus specimen, which according to our analyses has to be classified as C. medius. This should be regarded with caution. Our closest field sampling site to the Ambositra site is Ankazomivady (17), which is located about $30 \mathrm{~km}$ south of the town of Ambositra. The individuals caught here were classified as C. crossleyi and in the analyses were neither found to cluster with the C. minusculus specimen, nor with other C. medius individuals. Genetic analyses of the C. minusculus holotype, housed at the NHM, could possibly clarify the status of this individual. Cheirogaleus crossleyi individuals were found in the Northwest $(3,4,5,6)$ and at the previously mentioned Ankazomivady site (17) in the central highlands. The latter sampling site is not within the previously accepted distribution range of this species, but is concordant with the proposed distribution of this species by Hapke et al. (2005). Cheirogaleus major specimens were found 
from the southern most tip (20, 21; Fort Dauphin region) along the east coast (19, 18, 15, 12, $11,10,8)$ up to the northernmost site at Maroantsetra (7). These sampling sites are within the expected range for this species. Unfortunately this study could not confirm the presence of C. major in central western Madagascar, as reported by Thalmann and Rakotoarison (1994), Ausilio and Raveloanrinoro (1998) and Thalmann (2000).

The presence of four sympatric Cheirogaleus species in the region of Makira, in northeastern Madagascar as reported by Rasolofoson et al. (2007) is not concordant the results of this study. No more than three sympatric species per individual sampling site were found within the region of Makira. Either the reported species were actually, C. major, C. crossleyi, and C. medius, or a new species is present at this site.

\subsection{Conclusion}

This study did not entirely confirm Groves' taxonomy. We conclude that in our sample there are three distinct morphs/species of dwarf lemur that correspond to C. medius, C. major and C. crossleyi. Other data, including genetic data, are needed to verify this classification and justify the species level delimitation. According to the analyses presented here, C. adipicaudatus is synonymous with $C$. medius and $C$. ravus is synonymous with $C$. major. The sample size for $C$. minusculus is too small for decisive inferences. C. sibreei could represent a distinct morph, i.e. species, but further sources of corroborative data are required for a species level delimitation.

The mainly western geographical distribution of $C$. medius is extended by the sampling site Sambava on the northeastern coast. The Ankazomivady sampling site confirmed that $C$. crossleyi's distribution is not restricted to the northeast, as already pointed out by Hapke et al. (2005). No unexpected sampling sites were found for C. major and the previously accepted distribution range along the east coast remains valid.

This study has revealed lower diversity and a lower number of species of dwarf lemurs than expected. Especially in comparison to the closely related mouse lemurs (genus Microcebus) this seems surprising and an explanation for this difference is still lacking. In terms of conservation, this result implies that the limited funds available, can be focused on three species instead of seven. Furthermore, this study delivered important baseline data for conservation efforts in form of distributional data for the three species.

Further efforts in assessing the diversity of dwarf lemurs should focus on the Makira region in northeastern Madagascar, where four sympatric species were reported and on sites in central western Madagascar where C. major or a similar morph has been reported to be present.

\section{Acknowledgements}

We thank Olga Ramilijaona and and Daniel Rakotondravony from the Département de Biologie Animale de l'Université d'Antananarivo, and the Comission Tripartite CAFF for their authorization and support of this study. Field work was carried out 
under permit $\mathrm{N}^{\circ}$ 95/MINEVEF.EF/SG/DGEF/DPB/SCBLF/RECH and $\mathrm{N}^{\circ} 21 / \mathrm{MIN}$ EVEF.EF/SG/DGEF/DPB/SCBLF/RECH granted from the Ministère de l'Environnement des Eaux et Fôrets. We thank Christiane Denys and Jacques Cuisin (MNHN, Paris), Chris Smeenk (Naturalis, Leiden), and Paula Jenkins and Richard C. Sabin (NHM, London), Robert Asher and Frieder Mayer (ZMB, Berlin) and Katrin Krohmann (Senckenberg, Frankfurt) for kindly granting us access to the collections of the respective museums. We thank Dave Weisrock for his help with the statistical package JMP. Colin Groves is gratefully recognized for his prompt assistance with questions relating to his 2000 paper. We are grateful to Laurie Godfrey for her comments on the interpretation of Principal Component Analysis. For assistance with GIS in creating the map of the sampling sites we thank Dietmar Zinner. This study was supported by the German Science Foundation (DFG KA 1082/8-1 and -2) and the German Primate Center (DPZ). 


\section{Species delimitation in endemic Malagasy dwarf lemurs (genus Cheirogaleus) based on mitochondrial and nuclear DNA}

Linn F. Groeneveld ${ }^{1} \&$ Dave Weisrock $^{2} \&$ Rodin Rasoloarison ${ }^{3} \&$ Anne D. Yoder $^{2} \&$ Peter M. Kappeler ${ }^{1,4}$

\footnotetext{
${ }^{1}$ Department of Behavioral Ecology and Sociobiology, German Primate Center, Göttingen, Germany

${ }^{2}$ Biology Department, Duke University, Durham, NC, USA

${ }^{3}$ Départment de Biologie Animale, Université d'Antananarivo, Antananarivo, Madagascar

${ }^{4}$ Institute of Zoology und Anthropology, University of Göttingen, Göttingen, Germany
}

to be submitted to BMC Evolutionary Biology 


\begin{abstract}
Background: Species are viewed as the fundamental unit in most subdisciplines of biology. To conservationists this unit represents the currency for global biodiversity assessments. Even though Madagascar belongs to one of the eight hottest hotspots of the world, the taxonomy of its charismatic lemuriform primates is not stable. Within the last 25 years the species numbers have more than doubled, with many newly described species found among the nocturnal and rather small-bodied taxa. We characterize the diversity of the dwarf lemurs (genus Cheirogaleus) and assess the status of the seven described species, based on mtDNA (cytb + cox2) and three nuclear markers (adora3, fiba and $v W F$ ).

Results: This study identified three distinct evolutionary lineages with in the genus Cheirogaleus. Population genetic cluster analyses revealed a further layer of resolution within the three lineages and identified two distinct sets of populations/individuals per lineage.

Conclusion: Based on the general metapopulation lineage concept and multiple concordant data sets, we were only able to verify the exclusivity of three of the seven recognized dwarf lemur species: C. major, C. medius and C. crossleyi. These three species were found to be genealogically exclusive in both mtDNA and nDNA loci and furthermore, they exhibit morphological distinguishability. The molecular and morphometric data support that $C$. adipicaudatus and $C$. ravus are synonymous with $C$. medius and $C$. major, respectively. No clear subdivision within the clusters/clades can be found, as to warrant subspecific status. C. sibreei falls into the mtDNA C. medius clade, but in morphological analyses the membership is not clearly resolved. We do not have sufficient data to assess the status of $C$. minusculus. We propose that ecological and more geographic data should be collected to confirm these results.
\end{abstract}

\title{
3.1 Background
}

In most biodiversity and conservation assessments species are the fundamental unit in which diversity is measured (e.g. Ryder, 1986, Moritz, 1994). Depending on the criteria used to recognize species, vastly different numbers and distributions can arise. The difference in species numbers when utilizing a phylogenetic species concept (e.g Cracraft, 1983, Nixon and Wheeler, 1990) versus a biological species concept (e.g. Mayr, 1947) can be substantial. Agapow et al. (2004) estimated a $48 \%$ increase in recognized species across a wide range of organisms (ranging from fungi to mammals) when using a phylogenetic species concept. Similarly, Zink (1996) proposed a doubling of known bird species, mostly due to the elevation of subspecies to full specific status. Such a drastic difference in species numbers would necessitate an extensive revision of most conservation measures. Furthermore, species are the fundamental unit of comparison in all subdisciplines within biology (e.g. de Queiroz, 2005). As such, robust measures of species delimitation and boundaries are crucial to understanding the evolution of organisms and how best to manage biodiversity in the face of increased anthropogenic pressure.

The lemuriform primates of Madagascar have undergone a recent explosion in species descriptions, with as many as 47 new species described in the last 25 years as a result of intensified field work, the incorporation of molecular data in the elucidation of previously cryptic species, and a paradigm shift in what we call a species (Isaac et al., 2004, Yoder et al., 2005, Tattersall, 2007, Yoder, 2007). This increase has come in the face of tremendous anthropogenic pressures, with Madagascar having just a fraction of its original native habitat remaining (Myers et al. 2000). 
Increased lemuriform species diversity has been particularly acute in the family Cheirogaleidae, a clade of small-bodied and nocturnal lemurs with a generally cryptic morphology. In just over 10 years the number of recognized cheirogaleid species has more than quadrupled with most of this activity occurring in mouse lemurs of the genus Microcebus (e.g. Schmid and Kappeler, 1994, Zimmermann et al., 1998, Rasoloarison et al., 2000, Kappeler et al., 2005, Andriantompohavana et al., 2006, Louis et al., 2006, Olivieri et al., 2007). The dwarf lemur genus Cheirogaleus has received considerably less systematic attention despite having an island-wide distribution and sharing similar habitats with mouse lemurs. After a turbulent taxonomic past, this genus consisted of two species from the 1930s until the turn of the last century, with only the number of recognized subspecies varying between authors. It was proposed that a grayish colored species, Cheirogaleus medius, inhabited the western dry forests, and a larger rufus-colored form, Cheirogaleus major, occupied the eastern rainforests (Schwarz, 1931, Petter et al., 1977, Tattersall, 1982, Groves, 2000a). Using descriptive morphological assessments of existing museum material, Groves (2000a) split C. medius into two species: (1) C. medius in western Madagascar and (2) C. adipicaudatus in the south. Cheirogaleus major was split into five species: (1) C. major with a broad eastern distribution, (2) C. crossleyi, which is found more inland than C. major and also extends further north, (3) C. minusculus, known only from a single eastern locality at Ambositra, (4) C. ravus, which has a narrow coastal range within C. major, and (5) C. sibreei, with an unclear distribution, but known from an eastern locality at Ankeramadinika and possibly from the northwest at the Ampasindava Bay. While keeping these two groups (medius and major), Groves noted that he did so only for convenience. Nonetheless, he found these seven taxa to represent distinguishable morphs and interpreted them as separate genetic entities. While not explicitly stated in Groves (2000a), these seven taxa represent phylogenetic species, although it is important to point out that no objective criteria were used to diagnose these species as lineages or genetically distinct clusters.

Only one study has assessed the geographic patterning of genetic variation in Cheirogaleus. Hapke et al. (2005) used mitochondrial DNA (mtDNA) sequence data in an attempt to clarify the species status of three different morphotypes resembling C. crossleyi, C. major, and C. medius found in close proximity in the Fort Dauphin area of southeastern Madagascar. Using dense sampling in this area along with representatives of C. crossleyi, C. major, and C. medius from other portions of the island, Hapke et al. (2005) resolved three mtDNA haplotype clades each exclusive to one of the three representative species. These results are the only evidence to date that some of the species described by Groves (2000a) represent independent lineages. They also greatly expand the potential range of $C$. crossleyi into the southern portion of the island. Evidence for the exclusivity of C. adipicaudatus, C. minusculus, C. ravus, and C. sibreei is still lacking.

Robust studies of species delimitation should take into account both geographic and genetic variation in the recognition of species-level lineages. Field sampling of individuals should be sufficient to characterize the frequency of alleles within a single locality and also sufficient to characterize their spatial distribution. Genetic sampling should be sufficient to provide some 
understanding of the genealogical variation that exists across independent loci as a result of the lineage sorting process and gene flow. Recent efforts in lemur species delimitation have raised concerns regarding the methods and data used for the diagnosis of species-level lineages (Tattersall, 2007). The majority of recent descriptions have relied almost exclusively on mtDNA, using either genetic distances or fixed substitutions as criteria for species recognition or have not provided proper holotypes (e.g. Andriantompohavana et al., 2006, Louis et al., 2006, Olivieri et al., 2007). These practices beg the question whether such data and their analysis are sufficient to reliably diagnose species-level units, despite the potential for gene tree - species tree discordance due to gene flow or lineage sorting (Maddison, 1997, Nichols, 2001, Degnan and Rosenberg, 2006).

In this study we aim to provide a more comprehensive assessment of species diversity in the genus Cheirogaleus using an expanded geographic and genetic sampling approach. We use a concordance approach across independent sources of mitochondrial and nuclear DNA sequence data, complemented with morphological data, to identify independently evolving lineages according to the General Lineage Concept of species (de Queiroz, 1998, 2005). We also explore a finer level of resolution using population-genetic structuring methods to diagnose sets of populations that are genetically distinct and, which may represent more recently diverged, but independently evolving population-level lineages.

In our molecular analyses we included field samples, museum samples and already published sequences from GenBank. The morphological data are discussed in detail in chapter 2 and will here only be touched upon briefly. With this multilocus data set, complemented with morphological data, we aim to provide the best estimate of diversity in the genus Cheirogaleus currently possible and test the exclusivity of the seven recognized species. If we view taxonomic classifications as scientific hypotheses that may be refined and revised with new data (Groves, 2000b, 2001, Hey et al., 2003), our study can contribute significantly towards clarification and interpretation of dwarf lemur diversity.

\subsection{Material and methods}

\subsubsection{Sampling}

Field samples from a total of 48 individuals across 14 localities in Madagascar were collected between March 2003 and May 2007 (Table 3.1. Fig. 3.1). A maximum of three individuals per site, amounting to 31 individuals, were sacrificed and preserved as morphotypes. Tissue samples from internal organs (liver, kidney and spleen) and muscle tissue were stored in $70 \%$ EtOH. An additional 17 individuals were caught using Sherman live traps. One hundred traps were set along two to three transects for an average of 11 nights per site and baited with banana. Tissue for molecular analyses of these individuals was obtained by ear clipping after animals were anesthetized with GM2 (Rensing, 1999). External morphological measurements were taken from 44 individuals, while internal measurements were only available for the 31 morphotypes (see chapter 2). Animals were released at the site of capture at dusk on the 
following day.

Table 3.1: Field samples included in this study. Unique identifier, sampling locality, coordinates in decimal degrees, and number of sampling locality as marked on the map in Fig. 3.1. are given.

\begin{tabular}{|c|c|c|c|c|}
\hline Unique identifier & Locality & Latitude & Longitude & Locality \# \\
\hline E1001 & Ambanja/Ambato & -13.39583 & 48.47051 & 4 \\
\hline E1002 & Kirindy & -20.07370 & 44.67567 & 37 \\
\hline E1003 & Kirindy & -20.07222 & 44.67468 & 37 \\
\hline E1004 & Kirindy & -20.07222 & 44.67468 & 37 \\
\hline E1055 & Bekaraoka & -13.10470 & 49.70740 & 7 \\
\hline RMR132 & Marolambo & -20.06022 & 48.18330 & 21 \\
\hline RMR133 & Marolambo & -20.06022 & 48.18330 & 21 \\
\hline RMR134 & Marolambo & -20.06022 & 48.18330 & 21 \\
\hline RMR135 & Marolambo & -20.06022 & 48.18330 & 21 \\
\hline RMR137 & Marolambo & -20.06022 & 48.18330 & 21 \\
\hline RMR139 & Tampolo & -17.28683 & 49.40877 & 16 \\
\hline RMR140 & Tampolo & -17.28683 & 49.40877 & 16 \\
\hline RMR141 & Tampolo & -17.28683 & 49.40877 & 16 \\
\hline RMR146 & Andrambovato/Oranjatsy & -21.49593 & 47.40180 & 25 \\
\hline RMR148 & Andrambovato/Ambalavero & -21.49645 & 47.44537 & 25 \\
\hline RMR149 & Andrambovato/Ambalavero & -21.49645 & 47.44537 & 25 \\
\hline RMR150 & Bemaraha & -19.10358 & 44.76747 & 38 \\
\hline RMR152 & Bemaraha & -19.10358 & 44.76747 & 38 \\
\hline RMR153 & Montagne d'Ambre & -12.47478 & 49.21845 & 6 \\
\hline RMR155 & Montagne d'Ambre & -12.47478 & 49.21845 & 6 \\
\hline RMR158 & Montagne d'Ambre & -12.47478 & 49.21845 & 6 \\
\hline RMR162 & Ambanja/Benavony & -13.71113 & 48.47992 & 3 \\
\hline RMR164 & Ambanja/Beandroana & -13.70298 & 48.50455 & 3 \\
\hline RMR166 & Sambava & -14.39940 & 50.17387 & 9 \\
\hline RMR167 & Sambava & -14.39940 & 50.17387 & 9 \\
\hline RMR168 & Sambava & -14.39940 & 50.17387 & 9 \\
\hline RMR169 & Sambava & -14.39940 & 50.17387 & 9 \\
\hline RMR170 & Sambava & -14.39940 & 50.17387 & 9 \\
\hline RMR171 & Sambava & -14.39940 & 50.17387 & 9 \\
\hline RMR172 & Sambava & -14.39940 & 50.17387 & 9 \\
\hline RMR173 & Sambava & -14.39940 & 50.17387 & 9 \\
\hline RMR174 & Sambava & -14.39940 & 50.17387 & 9 \\
\hline RMR175 & Sambava & -14.39940 & 50.17387 & 9 \\
\hline
\end{tabular}

continued on next page 


\begin{tabular}{lllll}
\hline Unique identifier & Locality & Latitude & Longitude & Locality \# \\
\hline RMR176 & Sambava & -14.39940 & 50.17387 & 9 \\
RMR177 & Sambava & -14.39940 & 50.17387 & 9 \\
RMR178 & Sambava & -14.39940 & 50.17387 & 9 \\
RMR179 & Manantenina & -14.49100 & 49.81145 & 10 \\
RMR180 & Manantenina & -14.49100 & 49.81145 & 10 \\
RMR181 & Manantenina & -14.47548 & 49.83905 & 10 \\
RMR182 & Manantenina & -14.47548 & 49.83905 & 10 \\
RMR183 & Manantenina & -14.47548 & 49.83905 & 10 \\
RMR184 & Manantenina & -14.47548 & 49.83905 & 10 \\
RMR193 & Ankazomivady & -20.77995 & 47.18198 & 23 \\
RMR194 & Ankazomivady & -20.77995 & 47.18198 & 23 \\
RMR196 & Ankazomivady & -20.77995 & 47.18198 & 23 \\
RMR201 & Ivorona & -24.82367 & 46.94870 & 28 \\
RMR205 & Ivorona & -24.82367 & 46.94870 & 28 \\
RMR212 & Manantantely & -24.98815 & 46.92212 & 30 \\
\hline & & & & \\
\hline
\end{tabular}

A total of 44 additional tissue samples were collected from specimens in three European museums: the Muséum National d'Histoire Naturelle, Paris (MNHN); the Naturalis - Nationaal Natuurhistorisch Museum, Leiden; and the Natural History Museum, London (NHM). These specimens are the same individuals studied in the taxonomic revisions of Groves (2000a). Small amounts of dried tissue were taken from skulls and in a few cases from skins. Of the 44 museum samples taken, we were able to include 17 in the final mtDNA analyses (Table 3.2. . The presumed sampling sites of museum samples are marked with triangles on the map in Fig. 3.1.

Table 3.2: Museum samples included in this study. Museum the specimen is housed at, catalogue number of the specimen, species label as recorded by the museum, unique identifier, locality of provenance as indicated by the museum catalogues, and locality number as used in Fig. 3.1 are given.

\begin{tabular}{llllll}
\hline Museum & Catalogue number & Species & Unique identifier & Locality & Locality \# \\
\hline MNHN & CG 1932-3364 & C. adipicaudatus & Mu1045A & $170 \mathrm{~km}$ East of Tulear & 34 \\
MNHN & CG 1932-3365 & C. adipicaudatus & Mu1032A* & $170 \mathrm{~km}$ East of Tulear & 34 \\
MNHN & CG 1932-3365 & C. adipicaudatus & Mu1046A & $170 \mathrm{~km}$ East of Tulear & 34 \\
MNHN & CG 1967-1655 & C. medius & Mu1042A & Ampijoroa & 39 \\
MNHN & CG 1932-3362 & C. major & Mu1044A & Maroantsetra & 12 \\
MNHN & CG 1964-72 & C. ravus & Mu1034A & Mahambo & 17 \\
\hline
\end{tabular}

continued on next page 


\begin{tabular}{llllll}
\hline Museum & Catalogue number & Species & Unique identifier & Locality & Locality \# \\
\hline MNHN & CG 1964-74 & C. ravus & Mu1033A & Ambodivoangy & - \\
Naturalis & $1887: 66 \mathrm{~b}$ & C. sibreei & Mu1014A & Baie de Passandava & 2 \\
Naturalis & D.C. van Dam e & C. medius & Mu1020A & Mouroundava & 36 \\
Naturalis & D.C. van Dam a & C. medius & Mu1015A & Mouroundava & 36 \\
Naturalis & $1887: 66 \mathrm{f}$ & C. major & Mu1022A & Passumbée & 15 \\
Naturalis & $1887: 66 \mathrm{~g}$ & C. major & Mu1011A & Maranzettra & 12 \\
Naturalis & $1887: 66 \mathrm{c}$ & C. major & Mu1012A & Madagascar & - \\
NHM & 1948.160 & C. crossleyi & Mu1050A & Lake Alaotra & 14 \\
NHM & 1935.1 .8 .168 & C. adipicaudatus & Mu1051A & Tabiky & 35 \\
NHM & 1939.1289 & C. crossleyi & Mu1053A & Imerina, E. & 22 \\
NHM & 1935.1 .8 .169 & C. major & Mu1054A & Maroantsetra & 12 \\
\hline
\end{tabular}

MNHN $=$ Muséum National d'Histoire Naturelle

Naturalis Nationaal Natuurhistorisch Museum

NHM $=$ Natural History Museum

* same individual as Mu1046A

A total of 24 published Cheirogaleus haplotypes were incorporated into the analyses of the mtDNA data set (Table 3.3). Sequences from Mirza zaza, Microcebus berthae, M. murinus, and $M$. ravelobensis, which serve as representatives of other major cheirogaleid lineages (Horvath et al. 2008), were used as outgroups to root the phylogenetic trees. All previously published GenBank sequences are listed in Table 3.3 and the sampling localities for Cheirogaleus sequences are marked with squares on the map in Fig. 3.1.

Table 3.3: GenBank samples included in this study. GenBank accession numbers, species label as indicated in GenBank, locality of provenance and or unique identifier of the individual, locality number as used in Fig. 3.1, locus and number of basepairs available for the respective locus are given.

\begin{tabular}{llllll}
\hline GBAN & Species & Locality and/or unique identifier & Locality \# & Locus & Number of bp \\
\hline AH014105 & C. major & Nosy Boraha, Ile Ste. Marie & 13 & $c y t b$ & $208+259+241$ \\
AH014106 & C. major & Mahanoro & 20 & $c y t b$ & $633+241$ \\
AY441457 & C. major & Andasibe; JP118 & 19 & $c y t b$ & 1140 \\
AY584486 & C. medius & Manongarivo & 1 & $c o x 2$ & 684 \\
AY584487 & C. major & Ranomafana & 24 & $c o x 2$ & 684 \\
AY605903 & C. medius & Morondava CFPF & 37 & $c y t b$ & 1140 \\
AY605904 & C. medius & Foret de l'Ankarana & 5 & $c y t b$ & 933 \\
AY605905 & C. medius & Ste. Luce & 26 & $c y t b$ & 1140 \\
AY605906 & C. medius & Ste. Luce & 26 & $c y t b$ & 1140 \\
AY605907 & C. medius & Ste. Luce, Mandena & 26,29 & $c y t b$ & 1140 \\
AY605908 & C. medius & Mandena & 29 & $c y t b$ & 1140 \\
\hline
\end{tabular}




\begin{tabular}{|c|c|c|c|c|c|}
\hline GBAN & Species & Locality and/or unique identifier & Locality \# & Locus & Number of bp \\
\hline AY605909 & C. medius & Petriky, Lavasoa & 32,33 & cytb & 1140 \\
\hline AY605910 & C. medius & Lavasoa & 33 & $c y t b$ & 1140 \\
\hline AY605911 & C. major & Maroantsetra & 11 & $c y t b$ & 1140 \\
\hline AY605915 & C. major & Toamasina/Tamatave & 18 & cytb & 1140 \\
\hline AY605918 & C. major & Andohavondro & 31 & $c y t b$ & 1140 \\
\hline AY605919 & C. major & Manantantely & 30 & cytb & 1140 \\
\hline AY605920 & C. major & Manantantely, Mandena & 30,29 & $c y t b$ & 1140 \\
\hline AY605921 & C. major & Ivorona & 28 & $c y t b$ & 1140 \\
\hline AY605922 & C. major & Farafara & 27 & $c y t b$ & 1140 \\
\hline AY605923 & C. major & Farafara & 27 & $c y t b$ & 1140 \\
\hline AY605926 & C. crossleyi & Iharana/Vohemar & 8 & cytb & 633 \\
\hline AY605927 & C. crossleyi & Lavasoa & 33 & $c y t b$ & 1140 \\
\hline EF122247* & C. medius & Ampijoroa & 39 & cox2 & 529 \\
\hline EF122249 & C. medius & Ampijoroa & 39 & cytb & 307 \\
\hline AF285543 & Microcebus berthae & $\operatorname{Jorg} 46$ & - & $c y t b$ & 1140 \\
\hline AF285507 & Microcebus berthae & $\operatorname{Jorg} 46$ & - & $\operatorname{cox} 2$ & 684 \\
\hline AF285530 & Microcebus ravelobensis & RMR53 & - & $c y t b$ & 1140 \\
\hline AF285494 & Microcebus ravelobensis & $R M R 53$ & - & $\operatorname{cox} 2$ & 684 \\
\hline AF 285564 & Microcebus murinus & RMR24 & - & $c y t b$ & 1140 \\
\hline AF321177 & Microcebus murinus & RMR24 & - & $\operatorname{cox} 2$ & 684 \\
\hline EF052512 & Microcebus berthae & voucher 149 & - & adora3 & 370 \\
\hline DQ003347 & Microcebus berthae & voucher 149 & - & fiba & 605 \\
\hline EF052411 & Microcebus berthae & voucher 149 & - & $v W F$ & 773 \\
\hline EF052561 & Microcebus ravelobensis & voucher 66 & - & adora3 & 370 \\
\hline DQ003410 & Microcebus ravelobensis & voucher 66 & - & fiba & 605 \\
\hline EF052462 & Microcebus ravelobensis & voucher 66 & - & $v W F$ & 758 \\
\hline EF052619 & Microcebus murinus & voucher 203 & - & adora3 & 370 \\
\hline DQ003447 & Microcebus murinus & voucher 203 & - & fiba & 600 \\
\hline EF052508 & Microcebus murinus & voucher 203 & - & $v W F$ & 703 \\
\hline EU342234 & Mirza coquereli ${ }^{* *}$ & $D L C 2307$ & - & adora3 & 370 \\
\hline EU342261 & Mirza coquereli ${ }^{* *}$ & $D L C 2307$ & - & $f i b a$ & 603 \\
\hline AY434036 & Mirza coquereli ${ }^{* *}$ & $D U P C 384 F$ & - & $v W F$ & 756 \\
\hline U53571 & Mirza coquereli ${ }^{* *}$ & - & - & $c y t b$ & 1140 \\
\hline AY321460 & Mirza coquereli ${ }^{* *}$ & DUPC384F & - & cox2 & 684 \\
\hline
\end{tabular}

*same individual as EF122249

** listed in Genbank as M. coquereli, but have to be reclassified as Mirza zaza 


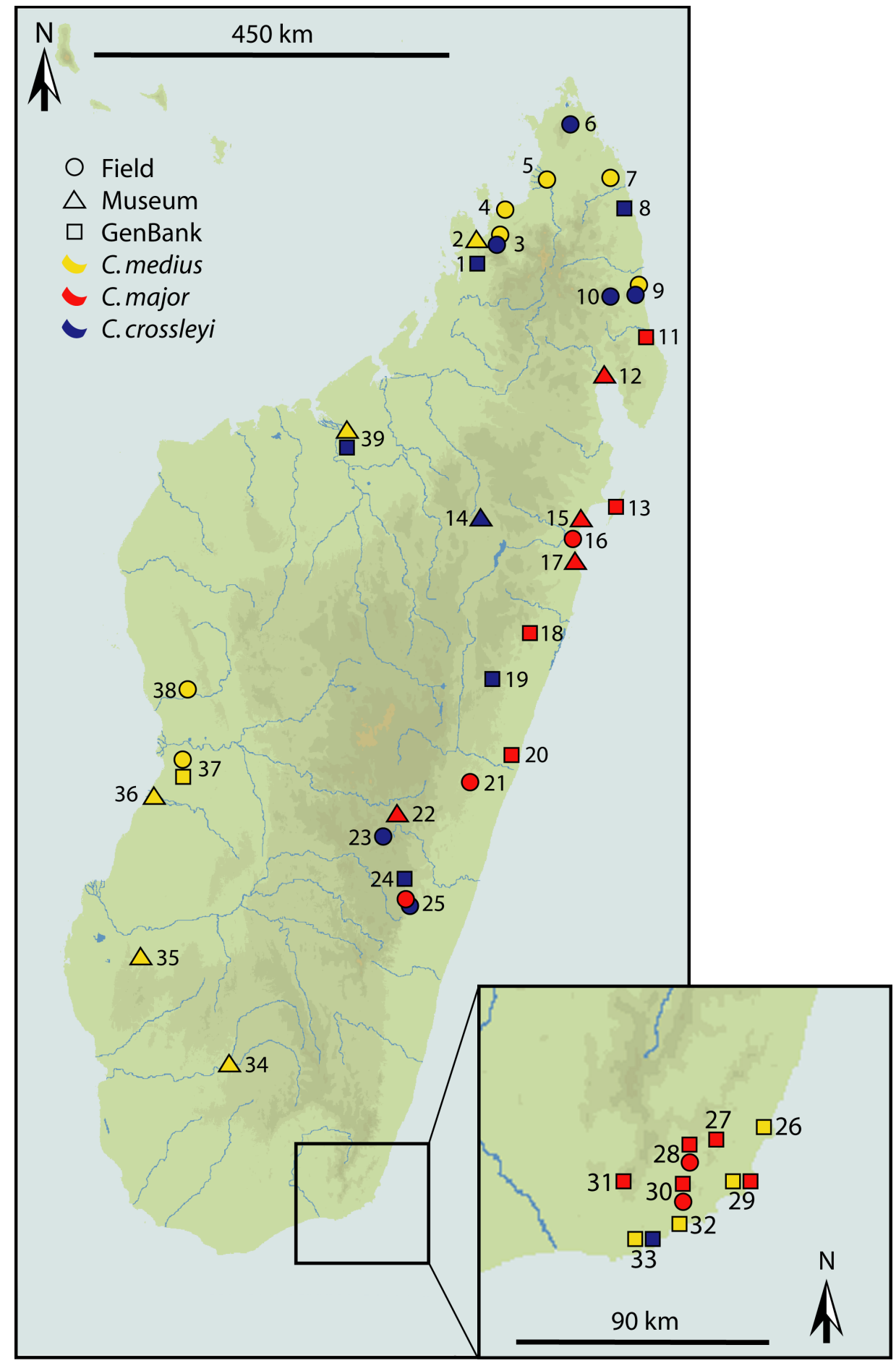

Figure 3.1: Sampling localities used in this study. Field samples collected by the authors are marked with circles. Presumed sites of origin for museum specimens are marked by triangles. Localities for GenBank samples are marked by squares. Symbols are colored according to the 3 main clades defined in the mtDNA gene tree (Fig. 3.2). More than one symbol can refer to one locality, if multiple species, or multiple types of data are found at one site. Detailed information for locality sites, marked by locality number, are given in Tables 3.1 3.3 and in Hapke et al. (2005). 


\subsubsection{Laboratory work}

Genomic DNA was extracted from both field and museum tissue samples using the QIAamp ${ }^{T M}$ DNA Mini Kit for DNA purification (Qiagen) following standard protocol. For the fieldcollected samples two mitochondrial [cytochrome b (cytb), cytochrome oxidase II (cox2)] and three nuclear loci [adenosine receptor A3 exon 2 (adora3), alpha fibrinogen intron 4 ( $f i b a$ ), and von Willebrand Factor intron $11(v W F)$ ] were amplified, using primers and annealing temperatures given in Table 3.4. Amplifications were either carried out in $10 \mu l$ reactions containing a final concentration of $0.25 \mu M$ of each primer, $3 \mathrm{mM} \mathrm{MgCl}_{2}, 0.25 \mathrm{mM} \mathrm{dNTPs,} 1 \mathrm{x}$ amplification buffer and $0.025 \mathrm{U} / \mu l$ taq (Jumpstart, Sigma) or in $30 \mu l$ reactions containing a final concentration of $0.33 \mu \mathrm{M}$ of each primer, $3 \mathrm{mM} \mathrm{MgCl}_{2}, 0.166 \mathrm{mM} \mathrm{dNTPs}, 1 \mathrm{x}$ amplification buffer and $0.033 \mathrm{U} / \mu l$ taq (Biotherm, Genecraft). Cycling conditions were 35 cycles of $30 \mathrm{~s}$ denaturation, $45 \mathrm{~s}$ annealing and 1 min elongation except for two loci. The adora3 fragment only needed 45 s elongation due to its short fragment length and the two overlapping cytb fragments were amplified using 1 min for all three steps. Amplification of the museum samples was carried out in $30 \mu \mathrm{l}$ reactions containing a final concentration of $0.33 \mu M$ of each primer, $2 \mathrm{mM} \mathrm{MgCl}_{2}, 0.166 \mathrm{mM}$ dNTPs, $1 \mathrm{x}$ amplification buffer and $0.033 \mathrm{U} / \mu \mathrm{l}$ taq (Biotherm, Genecraft). Cycling conditions were 50 cycles of 1 min denaturation, 1 min annealing and $30 \mathrm{~s}$ elongation. All amplifications of museum samples were verified by replication in a second independent lab. Wax-mediated Hot Start PCR was used on all $30 \mu l$ reactions to increase specificity and yield. PCR products were purified employing Montage ${ }^{\top M}$ PCR Centrifugal Filter Devices (Millipore) according to manufacturer's instructions. For a little more than half of the nuclear sequences, due to multiple polymorphic sites in heterozygous individuals, PCR products were cloned into a pGEM vector (pGEM ${ }^{\mathrm{TM}}$-T EasyVector System I, Promega), averaging 3 clones per polymorphic sequence. Both strands of all PCR products were sequenced, using the respective primer pair used for amplification and standard vector primers M13F and M13R for the cloned products, employing the BigDye ${ }^{\top M}$ Terminator v1.1 Cycle Sequencing Kit (Applied Biosystems) on an ABI PRISM ${ }^{\mathrm{TM}} 3100-$ Avant-Genetic Analyzer (Applied Biosystems).

\subsubsection{Phylogenetic analyses}

Sequences were edited and aligned using Sequencher 4.7 (Gene Codes Corporation, Ann Arbor, MI, USA) and manually checked by eye. Subsequently, sequences were collapsed into haplotypes using MACClADE 4.05 (Maddison and Maddison, 2002). Haplotype data sets were used for all subsequent Maximum Likelihood (ML) and Bayesian phylogenetic analyses. For all mtDNA analyses cytb and cox2 fragments were concatenated, resulting in an alignment of 1824 bp. GenBank, museum and field sample sequences were collapsed into haplotypes separately, since, due to missing data, unambiguous assignment was not possible. Uncorrected "p" distances for the cytb locus were calculated as implemented in PAUP* v4.0b10 (Swofford, 2002 .

Optimal nucleotide substitution models for each locus were chosen using the Akaike Infor- 
Table 3.4: Primers used in this study. The locus, primer name, primer sequence, reference and used annealing temperature in ${ }^{\circ} \mathrm{C}$ are given. The complete $c y t b$ locus was obtained by amplifying two overlapping fragments (primer pairs 1255, 2763 and 2695, 2510). The $c y t b$ fragments from museum material were amplified using primer pair 2877 , 2879 .

\begin{tabular}{|c|c|c|c|c|}
\hline Locus & Primer & Primer sequence $5^{\prime}-3^{\prime}$ & Reference & ${ }^{\circ} \mathrm{C}$ \\
\hline \multirow[t]{6}{*}{ cytb } & L-CYT & AAT GAT ATG AAA AAC CAT CGT TGT A & Roos (2003) & 55 \\
\hline & 2763 & GG(AG) ATT TT(AG) TCG GAG TCT GAT G & this study & \\
\hline & 2695 & CCG ATT CTT CGC ATT CCA CTT & this study & 55 \\
\hline & 2510 & GAC CAG (GT)GT ATT (AT)TT TAT ACT AC & C. Roos, pers. comm. & \\
\hline & 2877 & ACG TAA AC(CT) ACG GCT GAA & this study & 52 \\
\hline & 2879 & CCT CAG ATT CAT TCT ACT A & this study & \\
\hline \multirow[t]{2}{*}{ cox2 } & L7553 & AAC CAT TTC ATA ACT TTG TCA A & Adkins (1994) & 48 \\
\hline & H8320* & CTC TTT AAT CTT TAA CTT AAA AG & Adkins (1994) & \\
\hline \multirow[t]{2}{*}{ adora3 } & adora3F & ACC CCC ATG TTT GGC TGG AA & Murphy et al. (2001) & 52 \\
\hline & adora3R & GAT AGG GTT CAT CAT GGA GTT & Murphy et al. (2001) & \\
\hline \multirow[t]{2}{*}{ fiba } & Fiba-F & AAG CGC AAA GTC ATA GAA AAA G & Heckman et al. (2007) & 56 \\
\hline & Fiba-R & CTA AAG CCC TAC TGC ATG ACC CT & Heckman et al. 2007) & \\
\hline \multirow[t]{2}{*}{$v W F$} & vWF-10 & GAG CTG GAT GTC CTG GCC ATC CAT GGC AAC & Mancuso et al. (1989) & 60 \\
\hline & vWF-8 & GAG TGC CTT GTC ACT GGT CAT CCC ACT TCA A & Mancuso et al. (1989) & \\
\hline
\end{tabular}

*slightly modified from Adkins and Honeycutt (1994), fourth base not present in original primer

mation Criterion (AIC) as implemented in Modeltest 3.7 (Posada and Crandall, 1998). All ML analyses were conducted using a genetic algorithm approach in GARLI v0.951 (Zwickl, 2006). In GARLI, only the model specifications settings were adjusted according to the respective data set; all other settings were left at their default value. Ten replicates were run for each data set to verify consistency in $\log$ likelihood $(\operatorname{lnL})$ scores and tree topologies. Maximum-likelihood bootstrap percentages (BP) were estimated in GARLI by performing 500 pseudoreplicate runs on each nuclear data set and 100 pseudoreplicates on the mtDNA data set. PAUP* v4.0b10 (PPC) (Swofford, 2002) was then used to calculate a majority-rule consensus for each data set.

Bayesian analyses were conducted on a concatenated $c y t b+$ cox2 mtDNA data set and on the individual nuclear loci using MrBAYEs v3.1.2 (Ronquist and Huelsenbeck, 2003, Huelsenbeck et al., 2001). For the mtDNA analyses, a partitioned analysis was performed treating the $c y t b$ and cox2 genes as separate partitions, each with their own DNA substitution models. In all analyses we used four Monte Carlo markov chains (MCMC) with the default temperature of 0.2. Analyses were run for ten million generations with tree and parameter sampling occurring every 100 generations. Flat priors were assumed for the model parameters including the proportion of invariable sites and the gamma shape parameter of rate variation among sites. The first $25 \%$ of samples were discarded as burnin, leaving 75,001 trees per run. The adequacy of this burnin and convergence of all parameters were assessed by examining the uncorrected potential scale reduction factor (PSRF) (Gelman and Rubin, 1992) as calculated by MrBAYES v3.1.2 (Ronquist and Huelsenbeck, 2003, Huelsenbeck et al., 2001), which should approach 1 
as runs converge and visual inspection of the trace of the parameters across generations using the software Tracer v1.3 (Rambaut and Drummond, 2005). Posterior probabilities (PP) for each split and a phylogram with mean branch lengths were calculated from the posterior density of trees using MrBAYES v3.1.2 (Ronquist and Huelsenbeck, 2003, Huelsenbeck et al., 2001). Phylogenetic trees were visualized using TREEEdiT v1.0a10 (Rambaut and Charleston, 2002) and FigTree v1.0 (Rambaut, 2006).

Statistical parsimony haplotype networks were constructed for each nuclear locus using the program TCS version 1.21 (Clement et al., 2000). A 95\% connection limit was used and gaps were treated as missing data.

\subsubsection{Population structure}

A Bayesian population assignment test implemented in Structure v2.2 (Pritchard et al., 2000) was used to infer population structure based on a combined genotypic matrix from all four loci (adora3, fiba, $v W F$, and mtDNA) and also on the three locus nuclear matrix. An admixture model was used with correlated allele frequencies and no linkage among loci. For each number of populations assumed ( $\mathrm{K}=1$ to $\mathrm{K}=10$ ) we performed 50 replicate runs. The fourlocus data set was run with a burnin of 500,000 MCMC generations and 4 million subsequent generations. The three locus nuclear data set was run with a burnin of 250,000 generations and 1 million subsequent generations. The adequacy of the burnin and subsequent length of the MCMC chain was checked visually by plotting the parameters $\alpha$ and the Ln against the number of generations. In order to detect the favored number of genetic groups, an ad-hoc statistic $\Delta \mathrm{K}$ (Evanno et al., 2005) was calculated. In addition, a pairwise comparison of the 50 runs for each K was carried out using the perl script Simcoeff (Rosenberg et al., 2002). This procedure is based on the estimated membership fractions generated by STRUCTURE for a given K. The similarity coefficient for a pair of structure runs reflects the proportion of identical membership of individuals assigned through the Monte Carlo process. The proportion of runs resulting in $95 \%$ of the coefficients being equal is used to assess the stability of the cluster allocations. The clustering pattern for the run with the highest probability (estimated $\log$ probability of the data) for $K=2$ to $K=6$ was visualized using Microsoft ${ }^{\circledR}$ Excel ${ }^{\circledR}$ v11.3.3. Membership coefficients with posterior probabilities under 0.05 were disregarded and proportionally added to the remaining membership coefficients. Therefore, in Figure 3.6 and B.4, some individuals show membership in fewer than K clusters.

\subsection{Results}

\subsubsection{Haplotype data}

The concatenated $c y t b$ and cox2 sequences from the 48 field samples amounted to $1824 \mathrm{bp}$ and contained no indels. There were 468 variable sites defining 29 haplotypes. A fragment of 246 bp was obtained from museum samples of 16 individuals. Among these 16 samples, there were 48 variable sites defining 9 haplotypes. 24 Cheirogaleus haplotypes from GenBank, consisting 
of 17 complete and 5 partial (307-933 bp) cytb sequences, and 2 complete and 1 partial (529 bp) cox2 sequences (Table 3.3), were aligned with the field and museum haplotypes resulting in an overall set of 62 haplotypes defined through 494 variable sites (Table 3.5).

Nuclear DNA sequence data were generated from the 48 field samples. In all individuals both alleles were scored, amounting to a data set of 96 sequences for each locus. Among Cheirogaleus samples, the 370 bp exonic adora3 fragment had 26 variable sites and 29 haplotypes. The $604 \mathrm{bp}$ intronic fragment fiba had 44 variable sites and 49 haplotypes. The 793 bp intronic fragment $v W F$ had 93 variable sites and 52 haplotypes (Table 3.5). The adora3 alignment contained no indels. Both the fiba and $v W F$ alignments contained a small number of 1-2 bp indels. In addition, the $v W F$ alignment contained indels of 19 and 242 bp in 6 and 3 individuals, respectively.

The $c y t b$, cox2, and adora3 loci were each found to best fit a general time-reversible (GTR) model according to AIC. The mtDNA loci were best fit to a model with a proportion of invariant sites (I) and gamma distributed rate heterogeneity $(\Gamma)$, whereas adora3 was best fit to a model with a proportion of invariant sites. A K81uf $+\mathrm{I}+\Gamma$ model was favored for the fiba locus, (analysed under a GTR $+\mathrm{I}+\Gamma$ model in Bayesian phylogenetic analyses). The $v W F$ locus was found to best fit an HKY $+\mathrm{I}+\Gamma$ model (Table 3.5).

Table 3.5: Data sets and nucleotide substitution models used in this study. The data set, respective alignment length including outgroup, number of sequences in data set excluding outgroup, number of haplotypes excluding outgroup, and number of variable sites excluding outgroup, when gaps are considered missing data, except for the mtDNA data set are given. Nucleotide substitution models for each data set, as used in ML analyses and in Bayesian analyses, are listed.

\begin{tabular}{lllllll}
\hline Data set & $\begin{array}{l}\text { Alignment } \\
\text { length }\end{array}$ & $\begin{array}{l}\text { \# of } \\
\text { sequences }\end{array}$ & $\begin{array}{l}\text { \# of hap- } \\
\text { lotypes }\end{array}$ & $\begin{array}{l}\text { \# of variable/ } \\
\text { parsimony } \\
\text { informative sites }\end{array}$ & Model ML & $\begin{array}{l}\text { Model } \\
\text { Bayesian }\end{array}$ \\
\hline cytb + cox2 & 1824 & 88 & 62 & $494 / 442$ & GTR $+\mathrm{I}+\Gamma$ & $\mathrm{GTR}+\mathrm{I}+\Gamma$ \\
adora3 & 370 & 96 & 29 & $26 / 17$ & $\mathrm{GTR}+\mathrm{I}$ & $\mathrm{GTR}+\mathrm{I}$ \\
fiba & 604 & 96 & 49 & $44 / 34$ & $\mathrm{~K} 81 \mathrm{uf}+\mathrm{I}+\Gamma$ & $\mathrm{GTR}+\mathrm{I}+\Gamma$ \\
$v W F$ & 793 & 96 & 52 & $93 / 77$ & $\mathrm{HKY}+\mathrm{I}+\Gamma$ & $\mathrm{HKY}+\mathrm{I}+\Gamma$ \\
\hline
\end{tabular}

\subsubsection{MtDNA gene tree}

Bayesian and ML analyses of the mtDNA data set resulted in congruent trees with three main clades (A, B and C in Fig. 3.2 that largely correspond to the three species (including subspecies) recognized prior to the taxonomic revisions of Groves (2000a). Clade A is strongly supported ( $\mathrm{ML} \mathrm{BP}=100$ and Bayesian $\mathrm{PP}=1.0$ ) and consists of haplotypes sampled from western Madagascar, the southeastern tip (Fort Dauphin region) and two sampling sites in the northeast (Fig. 3.1). All mtDNA sequences generated from museum samples of C. medius, C. adipicaudatus, and C. sibreei are placed in clade A.

Clade $\mathrm{B}$ is strongly supported ( $\mathrm{ML} \mathrm{BP}=99$ and Bayesian $\mathrm{PP}=1.0$ ) and is comprised of 

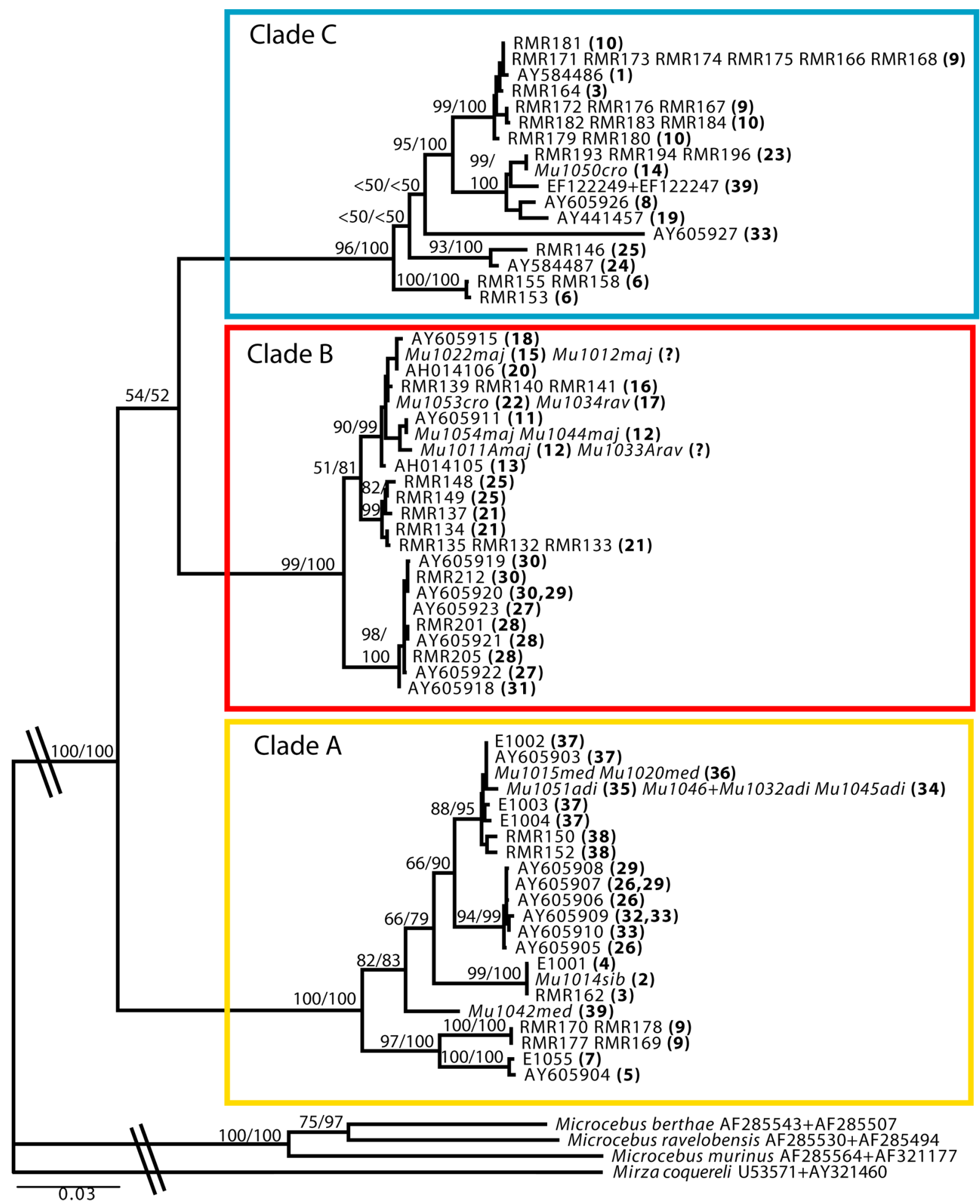

Figure 3.2: Maximum likelihood phylogram based on a total alignment of mtDNA cytb and cox2 haplotype sequences from field and museum samples (in italic) and of published GenBank samples. Tip labels contain the individual field numbers (E, RMR), the museum identifier, or GenBank accession number of sequences within a haplotype. The sampling locality a haplotype was found in, are given in bold type in parentheses, as marked in Fig. 3.1. GenBank haplotypes may occur in more than one locality. Maximum likelihood bootstrap values and Bayesian posterior probabilities are depicted above the branches. 
mtDNA haplotypes sampled from localities along the east coast from the southeastern tip (Fort Dauphin region) to the Maroantsetra peninsula in the northeast (localities 11 and 12). All mtDNA sequences generated from C. major and C. ravus samples fall into clade B. MtDNA sequence generated from a museum sample of $C$. crossleyi (locality 22) is also placed in Clade B.

Clade $\mathrm{C}$ is strongly supported ( $\mathrm{ML} \mathrm{BP}=96$ and Bayesian $\mathrm{PP}=1.0$ ) and also contains easternsampled haplotypes, ranging from the southeastern tip (Fort Dauphin region) up to the northern tip (Montagne d'Ambre, locality 6). Clade $\mathrm{C}$ also contains haplotypes sampled from three localities in the northwestern portion of the island (localities 1, 3, and 39). The sole museumgenerated sequence placed in Clade $\mathrm{C}$ is the C. crossleyi individual from locality 14.

The mtDNA-based relationships among clades A, B and C are poorly supported and are best viewed as unresolved. Uncorrected "p" distances based on the cytb locus (1140bp) were calculated for the three main clades (A, B and C). Distances between the three clades were fairly similar, with $11.4 \%$ between clades B and C, $12.6 \%$ between clades A and B and $13.10 \%$ between clades $\mathrm{A}$ and $\mathrm{C}$.

\subsubsection{Nuclear gene trees}

Bayesian, ML, and statistical parsimony analyses of the individual nuclear loci resulted in generally congruent gene trees with respect to the resolution of clades A, B, and C identified in the mtDNA gene tree. Bayesian and ML hierarchical gene trees are presented in supplementary Figs. B.1 B.3. Statistical parsimony haplotype networks are presented in Figs. 3.3 .3 .5$. The adora3 haplotype network (Fig. 3.3) resolves a clade of haplotypes corresponding to clade $\mathrm{C}$ in the mtDNA gene tree. The remaining adora3 haplotypes collectively correspond to clades A and B in the mtDNA gene tree. Shared polymorphism of adora3 haplotypes exists among these two mtDNA-based clades (Fig. 3.3). Adora3 haplotype 5 is found in individuals sampled from Ambanja (3), Ambato (4), and Kirindy (37) in the west (all containing Clade A mtDNA haplotypes), and is also sampled from Andrambovato (25) in the east (containing a Clade B mtDNA haplotype). Adora3 haplotype 2 is found in individuals sampled from Bemaraha (38) in the west (mtDNA Clade A) and in the Andrambovato locality (mtDNA Clade B).

The fiba haplotype network (Fig. 3.4) consists of two terminal clades that correspond to clades $\mathrm{A}$ and $\mathrm{C}$ in the mtDNA gene tree. An internal clade is also resolved corresponding to mtDNA clade B. All haplotypes in this latter clade have a common ancestor in fiba haplotype 8 found in a number of individuals sampled from Marolambo (21). These three clades are shallowly diverged from each other. Only 2 mutational steps separate sampled haplotypes in clades A and B. Only 4 mutations separate clades B and C.

The $v W F$ haplotype network (Fig. 3.5) consists of three clades of haplotypes that nearly completely correspond to clades $\mathrm{A}, \mathrm{B}$, and $\mathrm{C}$ in the mtDNA gene tree. The sole exception to this pattern is individual RMR149 from Andrambovato, which has a clade B mtDNA haplotype, but is homozygous for a "clade A" $v W F$ allele. 


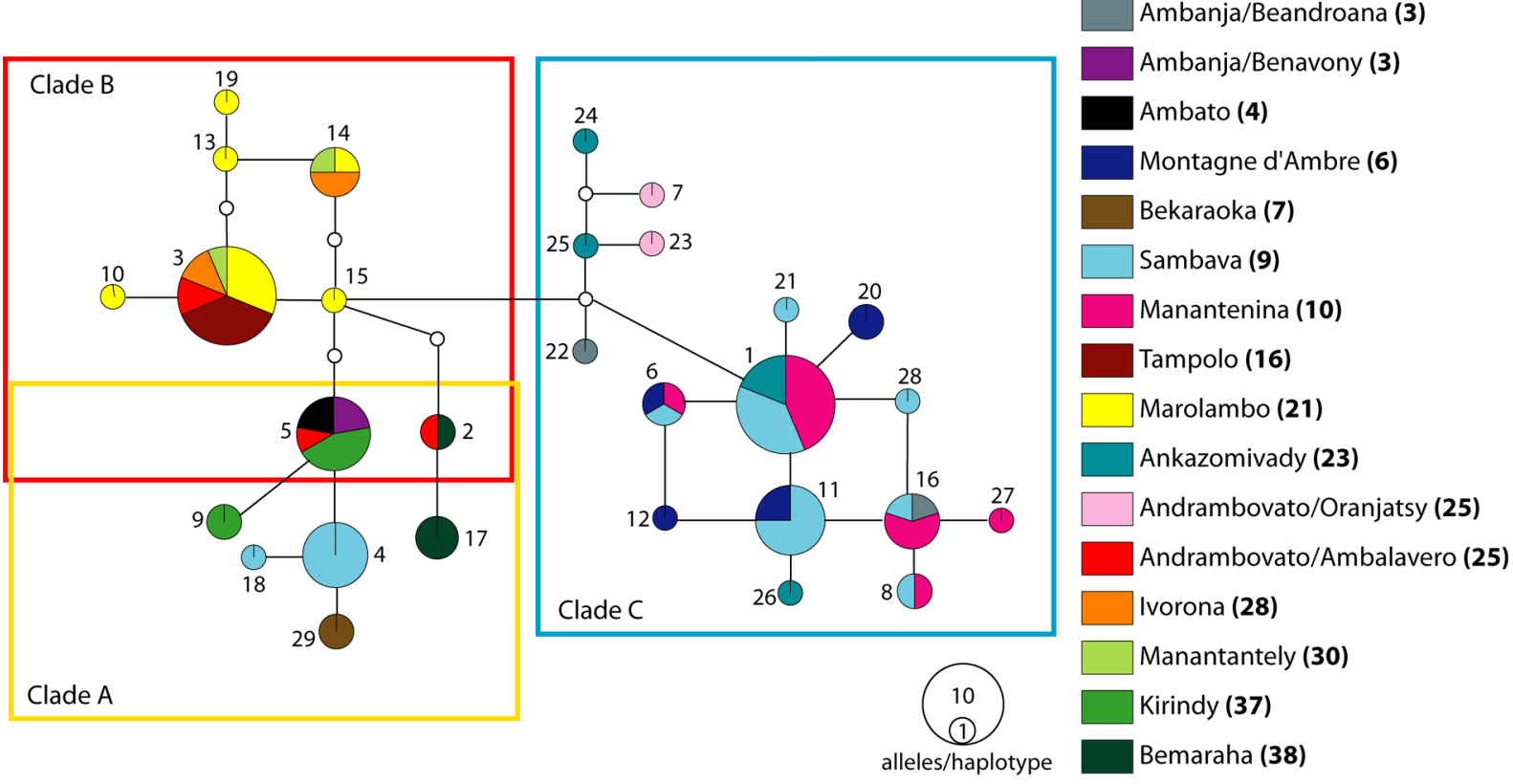

Figure 3.3: Statistical parsimony haplotype network representing the genealogical relationships among 29 haplotypes of the adora3 locus generated from field-collected samples. Haplotypes are colored according to the respective sampling locality, with the locality number given in the legend in bold as marked in Fig. 3.1. The size of a haplotype reflects the number of sequences that share a haplotype. Each of the haplotypes is numbered. Inferred intermediate haplotypes, either not sampled, or extinct, are represented by small non-colored circles. Groups of haplotypes found in individuals that correspond to clades A, B and C in the mtDNA tree are outlined by the colored frames.

\subsubsection{Population genetic clustering}

Bayesian population structure analyses of a combined mtDNA and nuclear data set and a data set comprised of only nuclear loci reveal very similar results (Fig. B.4), indicating that genetic structuring results are not being driven solely by the mtDNA data. Overall, differences between the results of the two data sets were only found in the number of identical solutions found for each $\mathrm{K}$ across replicates, in the exact contribution of each $\mathrm{K}$ to the genetic makeup of an individual and in the order that individuals split off to from a separate cluster at $\mathrm{K}=4$. At $\mathrm{K}>6$ the number of identical solutions plummets to 0 at a $95 \%$ threshold. A $\mathrm{K}=6$ is the favored solution according to the estimated ln probability of the data (mtDNA + nDNA: average $=-1033.2$, stdev $=2.4 ;$ nDNA: average $=-862.0$, stdev $=9.5)$, and according to the the ad-hoc statistic $\Delta \mathrm{K}$ (Evanno et al. 2005), which detected a clear mode at $\mathrm{K}=6$ for the calculations based on four loci, but showed no clear signal for the three nuclear loci. The $\mathrm{K}=6$ results from analyses of the combined nuclear data set are described below in the context of the three main mtDNA clades.

Most individuals possessing clade A mtDNA haplotypes are placed with high PPs in two distinct population clusters (depicted in green and purple in Fig. 3.6). The genetic compositions of individuals from Ambanja/Ambato (locality 4), Kirindy (37), and Ambanja/Benavony (3) 


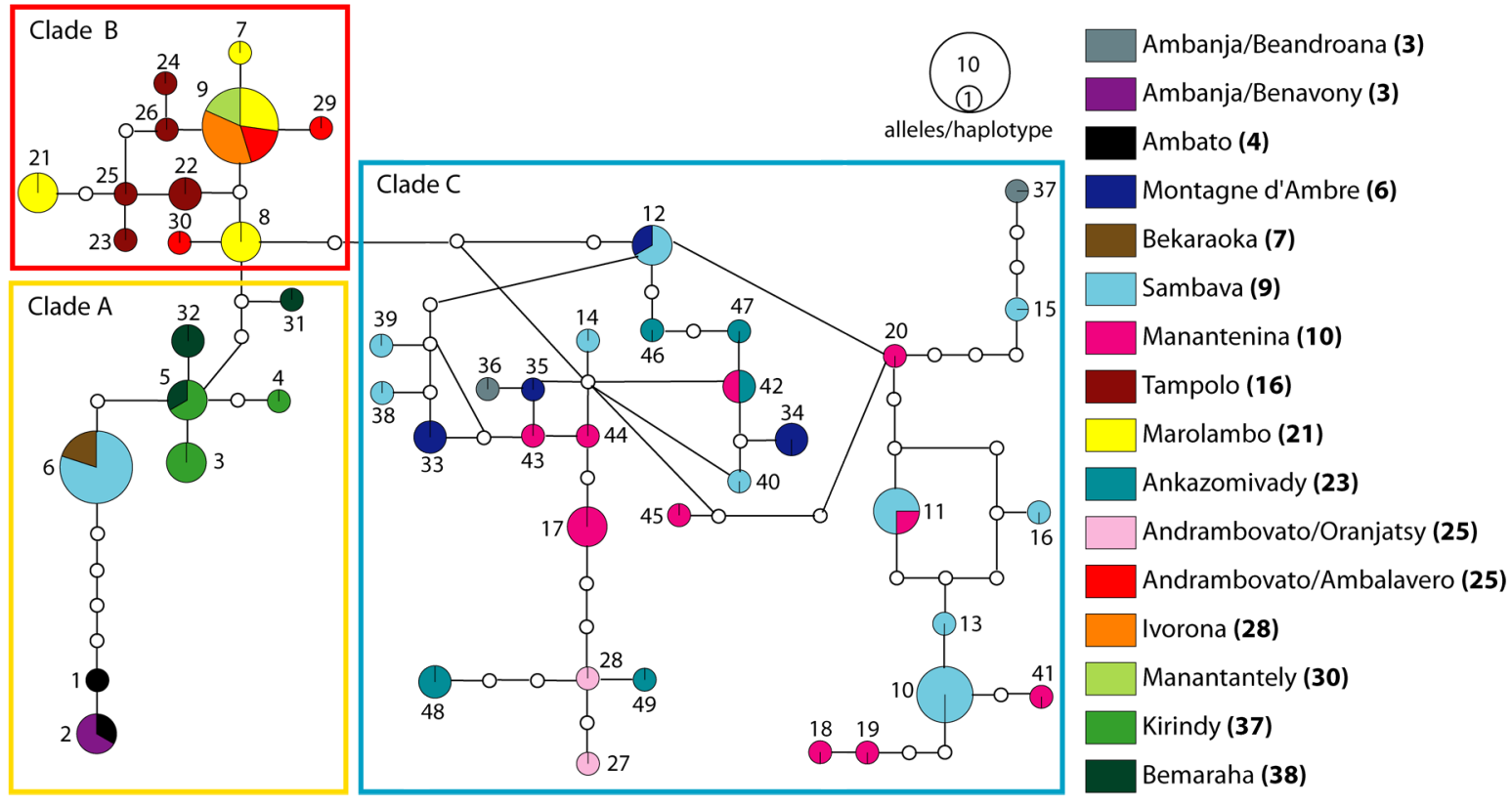

Figure 3.4: Statistical parsimony haplotype network representing the genealogical relationships among 49 haplotypes of the fiba locus generated from field-collected samples. Haplotypes are colored according to the respective sampling locality, with the locality number given in the legend in bold as marked in Fig. 3.1. The size of a haplotype reflects the number of sequences that share a haplotype. Each haplotype is numbered. Inferred intermediate haplotypes, either not sampled, or extinct, are represented by small non-colored circles. Groups of haplotypes found in individuals that correspond to clades A, B and C in the mtDNA tree are outlined by the colored frames.

are almost entirely of a single population cluster (green). A subset of individuals from Sambava (9) and the single individual sampled from Bekaraoka (7) are placed almost entirely in a second distinct population cluster (purple). Together with two individuals from Bemaraha (38), which do not fall into either of these two population clusters, the individuals forming these two distinct clusters correspond to clade A mtDNA haplotypes.

All individuals containing clade $\mathrm{C}$ mtDNA haplotypes are comprised of two population genetic clusters (depicted in blue and orange in Fig. 3.6). The remaining Sambava individuals are either completely comprised of, or contain high proportions of, a third population genetic cluster (orange) and low proportions of a fourth population genetic cluster (blue). This pattern is reversed in individuals sampled from Andrambovato/Oranjatsy (25), Montagne d'Ambre (6), Ambanja/Beandroana (3), Ankazomivady (23) and Manantenina (10).

Most individuals possessing Clade B mtDNA haplotypes constitute two population genetic clusters (depicted in red and yellow in Fig. 3.6). All individuals from Marolambo (21) and two individuals from Tampolo (16) are either completely comprised of, or contain some proportion of, a fifth population genetic cluster (red). The individuals sampled from Ivorona (28), Manantantely (30), Andrambovato,/Ambalavero (25) and the remaining individual from 


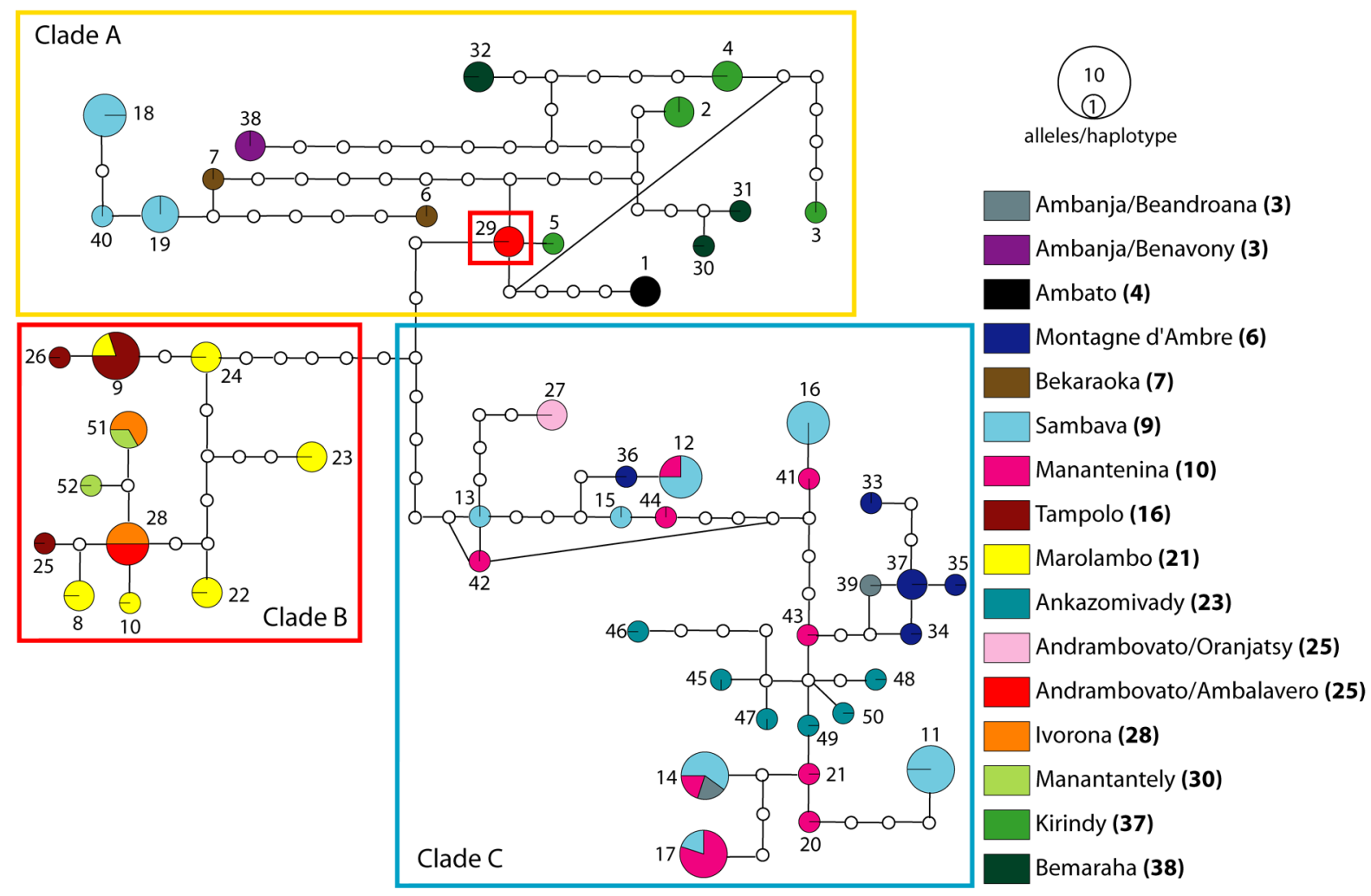

Figure 3.5: Statistical parsimony haplotype network representing the genealogical relationships among 52 haplotypes of the $v W F$ locus generated from field-collected samples. Haplotypes are colored according to the respective sampling locality, with the locality number given in the legend in bold as marked in Fig. 3.1. The size of a haplotype reflects the number of sequences that share a haplotype. Each haplotype is numbered. Inferred intermediate haplotypes, either not sampled, or extinct, are represented by small non-colored circles. Groups of haplotypes found in individuals that correspond to clades A, B and C in the mtDNA tree are outlined by the colored frames.

Tampolo (16) are placed entirely in a sixth cluster (yellow). The sole exception is one of the individuals from Andrambovato/Ambalavero (25), which is only placed in this cluster with a very low PP.

There is a clear indication that many individuals within mtDNA clades contain a mixed nuclear genetic composition. For example, more than half of individuals with clade $\mathrm{C}$ mtDNA haplotypes exhibit a genetic composition from two nuclear-defined clusters (orange and blue). This pattern also extends across mtDNA-defined clades. For example, some individuals from mtDNA clades A and B can contain a high proportion of a nuclear genetic cluster (blue) that is predominantly found in individuals with clade $\mathrm{C}$ mtDNA haplotypes. Overall, these patterns demonstrate the existence of two distinct nuclear genetic clusters within each mtDNA-based clade, but demonstrate the potential for extensive shared genetic makeup within and among these clades. 


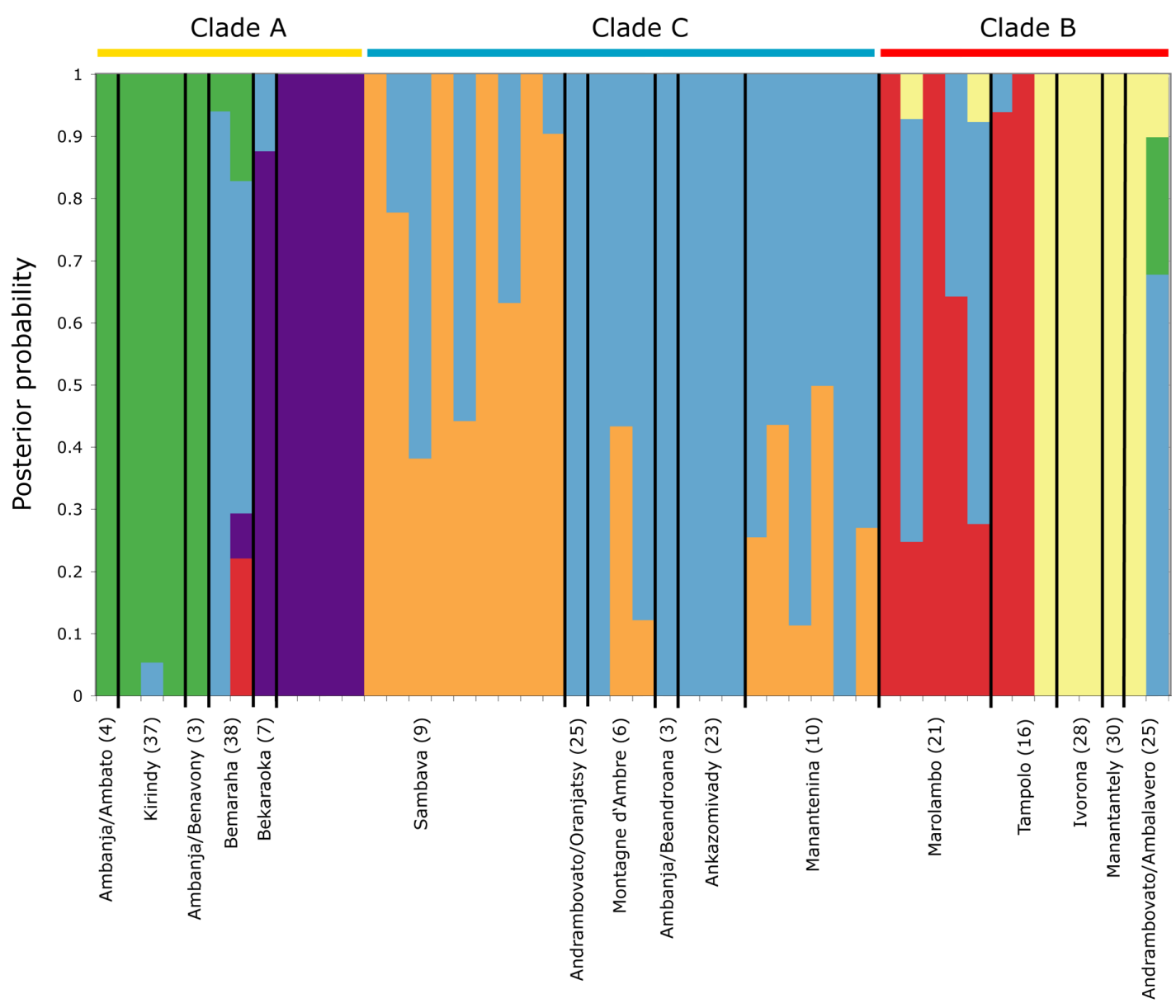

Figure 3.6: Bayesian assignment of the 48 field-collected individuals to populations, based on three nuclear loci, assuming a population number of $\mathrm{K}=6$. Individuals are arrayed along the $\mathrm{x}$ axis. The $\mathrm{y}$ axis denotes the cumulative posterior probability of an individuals placement in particular population(s). Individuals are divided into sampled populations by thin black lines. Sampled populations are labeled at the bottom with numbers in parentheses corresponding to the sampling locality as marked in Fig. 3.1 .

\subsection{Discussion}

Recently, species diversity in the genus Cheirogaleus has increased without the aid of genetic data in the diagnosis of independent evolutionary lineages. Groves (2000a) accepted seven Cheirogaleus species, based on morphological data, and interpreted them as separate genetic entities. In this study, mtDNA and nuclear gene sequences clearly resolved three main lineages within the genus Cheirogaleus. Using phylogenetic methods, no further monophyletic subdivisions based on mtDNA and nDNA could be resolved within each of the main lineages. However, a population genetic approach detected a further layer of differentiation within the three main evolutionary lineages and identified two genetically distinct population clusters within each main lineage. The three genealogically exclusive clades (A, B and C), are largely congruent with three clusters found according to morphometric data (chapter 2). This mor- 
phometric data set consisted of data collected from the individuals included in this study and museum specimens and can therefore be directly compared to the genetic data. Each genealogically exclusive clade will be discussed in detail in the following sections.

\subsubsection{Clade A}

Clade A is strongly supported in ML and Bayesian analyses of mtDNA sequence data. The mtDNA defined clade $\mathrm{A}$ is supported by nuclear data, although shared polymorphism of adora 3 and $v W F$ haplotypes among mtDNA-based clades $\mathrm{A}$ and $\mathrm{B}$ were detected. The nuclear markers used in this study, have been used to test the hypotheses of species identity and historical relationships of mouse lemurs (genus Microcebus), which were previously described according to mtDNA and morphometric data (Heckman et al. 2007). In this study four independently segregating nuclear loci (including the three nuclear loci used in this study) unanimously revealed three deeply diverged clades, but incomplete lineage sorting and low mutation rates limited the phylogenetic resolution of the nuclear data at a specific-level. The same processes could be responsible for the shared polymorphism of adora3 and $v W F$ haplotypes among mtDNA-based clades.

In chapter 2, species names were unambiguously assigned to the morphometric clusters. Clade A corresponds to C. medius, according to morphometric and geographic data, as well as the mtDNA sequences generated from museum samples of $C$. medius, which all fall into clade A.

MtDNA and morphological analyses of C. adipicaudatus specimens are congruent and invariably place these individuals into the $C$. medius cluster or mtDNA clade A, respectively. We would therefore propose that $C$. adipicaudatus is synonymous with $C$. medius.

The $C$. sibreei individual included in this study falls into the $C$. medius clade. In phylogenetic mtDNA analyses it even directly clusters with individual RMR162, with which it also clusters in morphological hierarchical cluster analyses. Albeit, the morphological analyses overall were inconclusive about the status of $C$. sibreei as a distinct morph.

\subsubsection{Clade B}

Clade B is strongly supported in ML and Bayesian analyses of mtDNA and corroborated by nuclear data, although shared polymorphism of adora3 and $v W F$ haplotypes among mtDNAbased clades $\mathrm{A}$ and $\mathrm{B}$ were detected. According to morphological analyses and mtDNA sequences generated from museum C. major specimens, clade B corresponds to C. major. MtDNA and morphological analyses of $C$. ravus specimens are congruent and place these individuals into the $C$. major cluster or mtDNA clade B, respectively. We therefore conclude that $C$. ravus is synonymous with $C$. major. We cannot conclude anything about the $C$. crossleyi museum specimen, which unexpectedly falls into clade $\mathrm{B}$, since this individual, due to being classified as juvenile, was not included in the morphological analyses. 


\subsubsection{Clade C}

Clade $\mathrm{C}$ is well supported in ML and Bayesian analyses of mtDNA and unambiguously corroborated by all three nuclear markers. Clade $\mathrm{C}$ corresponds to C. crossleyi, according to morphological and one mtDNA sequence from a museum C. crossleyi specimen (ZD.1948.160). The status of this specimen in morphological analyses was not fully resolved, but we concluded that it most likely represents a C. crossleyi individual (see chapter 2).

The one incongruence between morphological and molecular analyses found at the level of the three main clades, concerns the two individuals RMR146 and RMR164. According to morphological data they were classified as C. major individuals, but molecular analyses would label them as $C$. crossleyi. We propose that these individuals should be considered to be C. crossleyi.

\subsubsection{Comparison to Groves' taxonomy}

Our analyses did not entirely confirm Groves' taxonomy. We could only confirm the genealogical exclusivity of three of the species he accepted: C. medius, C. major and C. crossleyi. We propose, according to congruent morphological and mtDNA data, that $C$. adipicaudatus and C. ravus are synonymous with $C$. medius and C. major, respectively. According to our analyses there is no indication that $C$. sibreei represents a distinct lineage. We could not detect a clear membership of C. sibreei individuals in any one clade/cluster according to morphological or genetic data. We have no molecular data to assess the status of C. minusculus. The single specimen upon which this species was described, is listed as from Ambositra/Antsirabe in the museum catalogue of the British Museum of Natural History (today: Natural History Museum [NHM]) (Jenkins, 1987). Our geographically closest sampling site to this locality is Ankazomivady (locality 23), which is about $29 \mathrm{~km}$ south of the town of Ambositra. The Ankazomivady individuals, which according to morphometric and genetic analyses are C. crossleyi individuals, do not cluster with the C. minusculus individual in morphometric analyses. Thus, there is no indication that these individuals could represent C. minusculus. Unfortunately, the NHM will not allow sampling of holotype material for molecular analyses, which would be crucial to assess the status of this proposed species.

\subsubsection{Fine-scale subdivisions within the main lineages}

Within the three species-level distinct evolutionary lineages further genetic substructuring was detected via population genetic clustering. Especially the Sambava (locality 9) population exhibits a special status. The two species, C. medius and C. crossleyi, are found at this locality and for both species the individuals from Sambava form a distinct cluster separated from all other individuals of the respective species. In the case of $C$. medius the individual from the geographically close locality Bekaraoka (7) is included in this distinct 'Sambava' cluster.

Why there are cases where clusters contain multiple species, such as the $C$. medius individuals from Bemaraha (38), which cluster with C. crossleyi individuals, is not clear. Possibly 
this study included too few loci to obtain a fully resolved picture.

\subsubsection{Geographic distributions of the species}

The distribution of the three species can be extended by further sampling sites identified by this study (Fig. 3.1). All three species are present in forest fragments in the Fort Dauphin region (southeastern tip), as described by Hapke et al. (2005). The range of C. medius extends along the west coast up north to Ankarana (5). This species is however also found at two sites, Bekaraoka (7) and Sambava (9), on the northeastern coast. This is consistent with a presumed C. medius population at Daraina, northeastern Madagascar, as listed in Mittermeier et al. (1994). The distribution of C. medius is therefore not limited strictly to the western dry forests. The range of $C$. crossleyi extends from the southeastern tip all the way north to Montagne d'Ambre (6) with sampling sites never being found directly on the coast, but rather further inland along the eastern edge of the central plateau. There are a few exceptions in the north, both on the east and west coast: Sambava (9), Iharana/Vohemar (8), Ambanja/Beandroana (3), Manongarivo (1) and Ampijoroa (39). Cheirogaleus major is found, as previously described, in the eastern lowland forest, from the southeastern tip as far north as Maroantsetra (11). Additionally, there is one field (25) and one museum sampling site (22) along the eastern edge of the central plateau where C. major is found. We currently do not have any data to assess the populations mentioned in Thalmann and Rakotoarison (1994), Ausilio and Raveloanrinoro (1998), Thalmann (2000) and Rasolofoson et al. (2007).

\subsubsection{Multifaceted approach in species delimitation in lemurs}

This study has shown that multiple independent lines of data can yield a robust estimate of diversity. Morphological data alone would not have been able to uncover possible cryptic species or could also have led to an overestimation of species diversity as demonstrated for mouse lemurs (Heckman et al., 2006). Solely using mtDNA might have diagnosed distinct populations, or even local matrilines as species and only utilizing nuclear data would most likely underestimate diversity. Of course, it would be desirable to add more geographic and ecological information to the current data, in order to verify our conclusions. One starting point would be to examine the distinct populations identified through the population genetic cluster analysis, with special emphasis on the Sambava locality, where C. medius and $C$. crossleyi are found in sympatry. Furthermore, a direct comparison of the speciation patterns found in dwarf lemurs and those known for mouse lemurs, with estimates of time divergence dates, would be valuable to answer questions about the mechanisms driving this species radiation in Madagascar.

\subsection{Conclusion}

Based on the general metapopulation lineage concept and multiple concordant markers, we were only able to verify the exclusivity of three of the seven recognized dwarf lemur species: 
C. major, C. medius and C. crossleyi. These three species were found to be genealogically exclusive in both mtDNA and nDNA loci and furthermore, they exhibit morphological distinguishability. The molecular and morphometric data support that C. adipicaudatus and C. ravus are synonymous with $C$. medius and $C$. major respectively. No clear subdivision within the clusters/clades can be found, as to warrant subspecific status. C. sibreei falls into the mtDNA C. medius clade, but in morphological analyses the membership is not clearly resolved. We do not have sufficient data to assess the status of $C$. minusculus.

The conclusions of this study may not delight conservationists, as they often are keen, in the attempt to conserve all diversity, to present as many species as possible. Nonetheless, a robust estimate of species boundaries should also be in their interest, as the species category constitutes the basis of their work. For dwarf lemur conservation this study implies that, since there are fewer species with greater individual abundances and distributions, dwarf lemurs should be less threatened than previously thought. Whether this implies that dwarf lemurs are not suitable flagship species and need not be regarded as intensely as other lemur taxa, or whether more emphasis in conservation should be placed on other measures than species numbers (e.g as proposed by Sechrest et al., 2002) we leave to the conservationists to decide.

The concordance approach, based on multiple independent lines of data, yielded a robust estimate of diversity within the genus Cheirogaleus and we conclude that this approach is well-suited for species delimitations.

\section{Acknowledgements}

We thank Olga Ramilijaona and and Daniel Rakotondravony from the Département de Biologie Animale de l'Université d'Antananarivo, and the Comission Tripartite CAFF for their authorization and support of this study. Field work was carried out under permit $\mathrm{N}^{\circ}$ 95/MINEVEF.EF/SG/DGEF/DPB/SCBLF/RECH and $\mathrm{N}^{\circ} 21 / \mathrm{MIN}$ EVEF.EF/SG/DGEF/DPB/SCBLF/RECH granted from the Ministère de l'Environnement des Eaux et Fôrets. We thank Jacques Cuisin (MNHN, Paris), Chris Smeenk (Naturalis, Leiden), and Paula Jenkins and Richard C. Sabin (NHM, London) for kindly granting us access to the collections of the respective museums and we thank Steven Goodman for an additional tissue sample. We thank Christian Roos and Christina Oberdieck for their help in the lab. Gert Wörheide is gratefully recognized for providing lab space for the verification of the museum samples. Eildert Groeneveld is thanked for providing the computational resources needed for the MCMC-based analyses. For assistance with GIS in creating the map of the sampling sites we thank Dietmar Zinner. This study was supported by the German Science Foundation (DFG KA 1082/8-1 and -2) and the German Primate Center (DPZ). 


\title{
4 Dwarf lemurs in space and time: Phylogeography of the genus Cheirogaleus
}

\author{
Linn F. Groeneveld ${ }^{1} \&$ Rodin Rasoloarison ${ }^{2} \&$ Dave Weisrock $^{3}$ \& \\ Peter M. Kappeler ${ }^{1,4}$
}

${ }^{1}$ Department of Behavioral Ecology and Sociobiology, German Primate Center, Göttingen, Germany

${ }^{2}$ Départment de Biologie Animale, Université d'Antananarivo, Antananarivo, Madagascar ${ }^{3}$ Biology Department, Duke University, Durham, NC, USA

${ }^{4}$ Institute of Zoology und Anthropology, University of Göttingen, Göttingen, Germany

to be submitted to Journal of Biogeography 


\begin{abstract}
Madagascar is characterized by astonishingly high rates of endemism at all taxonomic levels. All primates found on this island are endemic and many genera show a distinct pattern of numerous local endemics restricted to very small areas. Little is known about the mechanisms that shaped the extensive radiations on this island. Two models exist that aim to explain the evolutionary history of speciation in Madagascar. The more recent model assumes that river catchments with sources at lower elevations were zones of isolation, leading to locally endemic species, whereas rivers with sources at higher elevations served as retreat and dispersion zones during Quaternary climatic shifts. The second model assumes that the limits of biogeographic zones based on phytogeography should correlate with distributional limits of terrestrial vertebrate taxa. The role of rivers as barriers to dispersal and as corridors facilitating dispersal are discussed in the context of both models. The assessment of biogeographic patterns is heavily influenced by changing taxonomies and revised phylogenies. Since new distributional data and a reassessed taxonomy and phylogeny are available for the dwarf lemurs (genus Cheirogaleus), we aim to test which model better explains the contemporary distribution of members of this genus. Furthermore, we add a historical perspective through time divergence estimates at the level of species within the Cheirogaleidae. For our analyses we combined field samples with museum specimens and genetic sequence data from GenBank. Neither one of the models is congruent with the distributional patterns found. The age estimates of species level divergences within the genus Cheirogaleus and the closely related genus Microcebus predate the Quaternary, and thus add further evidence that models relying on climatic shifts during the Pleistocene as the driving force for speciation are not adequate.
\end{abstract}

\title{
4.1 Introduction
}

Madagascar, the fourth largest island of the world, is found in the Indian Ocean off the east coast of Africa. Today it is separated by $300-450 \mathrm{~km}$ from Africa through the Mozambique Channel. Madagascar broke off mainland Africa with the Indian subcontinent attached to its eastern margin and Antarcto-Australia to the south, during the fragmentation of Gondwana around 165-150 mya. The Indian subcontinent detached around 88mya and rapidly drifted northeastwards. The dating of these events are relatively well documented and largely agreed upon in the scientific community (Rabinowitz et al., 1983, Storey et al., 1995, Krause, 2003). Less certain is how and when the southern landmasses separated from Madagascar; with the estimated timings ranging from 130-125 mya to as late as 80 mya (e.g. Roeser et al., 1996, Hay et al., 1999, Krause, 2003, Briggs, 2003). The break-up of Madagascar and the Indian subcontinent led to a drastic reduction of lowland area in Madagascar and explains Madagascar's current topography. The island is defined by a long chain of mountains that runs along its eastern edge. Towards the west the elevation drops slowly and vast lowland regions prevail, while the east is steeper with mountain rivers plunging to the sea after short distances. India is what would have been Madagascar's eastern lowland (e.g. Goodman and Ganzhorn, 2004ba).

Madagascar is characterized by its unique flora and fauna. High rates of endemism, especially at high taxonomic levels, coupled with extreme anthropogenic pressures make Madagascar a biodiversity hotspot (Myers et al., 2000). Species level endemism rates are estimated to be around $92 \%$ for vascular plants (excluding ferns), about $86 \%$ for macroinvertebrates and around $84 \%$ for land vertebrates. Of those land vertebrates, terrestrial animals exhibit the highest rates of endemism ranging from 92-100\% (Goodman and Benstead, 2005), which is 
mainly due to a low rate in colonization events with subsequent speciose endemic radiations (e.g. Yoder et al., 1996, Nagy et al., 2003, Yoder et al., 2003, Vences, 2004, Poux et al., 2005, Yoder and Nowak, 2006).

Throughout the last millennia Madagascar has, contrary to earlier prevailing views, experienced a dynamically changing climate in response to periods of glaciation and interglaciation. Along with climatic fluctuation came pronounced shifts in vegetational structure. Presettlement Madagascar was by no means a stable environment (e.g. Tattersall, 1982, Richard and Dewar, 1991, Burney, 1997, Burney et al., 2004, Goodman and Ganzhorn, 2004b). After human arrival on the island around 2000 years ago the original vegetation of the central highlands, in the Holocene most probably a mix of open country, woodland and forest, changed rapidly into grasslands through anthropogenic influences (Koechlin et al., 1974, Burney, 1987. Richard and Dewar, 1991, Goodman and Ganzhorn, 2004a). Today Madagascar's climate, due to the mountain chain intercepting the trade winds from the Indian Ocean, is characterized by a steep rainfall gradient from the northeast to the southwest of the island.

In terms of phytogeography Madagascar has traditionally been divided into a dry western and a humid eastern zone (Humbert, 1955, Koechlin et al., 1974, Du Puy and Moat, 1995). Humbert (1955) defined six phytogeographic zones, which formed the basis for subsequent analyses of concordance among modern plant and vertebrate communities (Petter et al., 1977. Tattersall, 1982, Richard and Dewar, 1991, Martin, 1995, Yoder et al., 2000, Pastorini et al., 2003, Ganzhorn et al., 2006, Olivieri et al., 2007). A number of taxa have shown discordance with this biogeographic hypothesis based on phytogeography, which stresses the difference between the western dry and eastern humid habitats. The prevailing view that the east and west differ markedly may be artificially accentuated through the central highlands having been cleared of most of their original vegetation within the last 2000 years. Thus, intermediate vegetation forms that constituted a link between east and west no longer exist. During the last decades information suggesting that there actually is a pronounced east-west connectivity has accumulated (Tattersall, 1982, Thalmann and Rakotoarison, 1994, Martin, 1995, Ganzhorn, 1998, Yoder et al., 2000, Ganzhorn et al., 2006).

In this biogeographical hypothesis not only the central highlands, but also major rivers separating the different zones, were proposed to act as dispersal barriers. Recent research has shown that some rivers with sources at high elevations may indeed serve as barriers to dispersal (north-south), but that rivers with sources at lower elevations may be circumvented by species with distributions above the elevation of the source. Furthermore, it is unclear how stable the courses of these rivers have been over time. In contrast to viewing rivers as barriers, they have also been discussed to serve as corridors, facilitating dispersal. During Pleistocene droughts forests along rivers could have served as refugia for forest species. Since the sources of rivers draining eastwards and westwards are in numerous cases within close distance of each other, they could have served as corridors for exchange of species between east and west (Tattersall, 1982, Goodman and Ganzhorn, 2004a, Ganzhorn et al., 2006).

These ideas and current research on the distributions of extant species led to a new biogeographical hypothesis: the 'centers-of-endemism' hypothesis. The main assumptions are 
that during the periods of cooler and drier climate in the Quaternary, river catchments with sources at low elevations $(<2000 \mathrm{~m})$ were zones of isolation and led to locally endemic species, while rivers with sources at higher elevations acted as zones of retreat and dispersal (Wilmé et al., 2006).

The assessment of biogeographic patterns is heavily influenced by changing taxonomies and revised phylogenies (Richard and Dewar, 1991). Due to the reassessed taxonomy of the genus Cheirogaleus (see chapter 2 and 3 ) and the availability of new distributional data, we aim to test which of these two biogeographic hypotheses (biogeographic zones based on phytogeography and 'centers-of-endemism') better explains the contemporary distribution of the members of the genus Cheirogaleus. Since neither one of these hypotheses is based on Cheirogaleus distributional data, our analyses constitute an independent test of the applicability of the respective hypothesis. Furthermore, we add a historical perspective through time divergence estimates at the level of species within the Cheirogaleidae. For our analyses we combined field samples with museum specimens and genetic sequence data from GenBank.

\subsection{Material and methods}

\subsubsection{Concordance with biogeographic hypotheses}

A total of 113 Cheirogaleus individuals from 39 localities in Madagascar formed the basis of the first part of this study. Of those 113 individuals, 48 were obtained from 14 localities in the field, 14 were obtained from European museum collections from additional 10 localities and 51 were acquired from GenBank from 15 additional localities (Table 4.1). Detailed information on the samples is presented in chapters 2 and 3 . In this study we assumed the taxonomy as assessed in chapters 2 and 3 .

The relationships among the 113 individuals assumed in this study are based on a phylogeny reconstructed from $1824 \mathrm{bp}$ of mtDNA $(c y t b+c o x 2)$ as described in detail in chapter 3. Fig. 3.2. Simplified versions of this phylogeny (Fig. 4.1) were superimposed on a map of centers of endemism and retreat-dispersion watersheds as defined by Wilmé et al. (2006) and were used for reconstructing ancestral distributions for each node using dispersal-vicariance analysis (DIVA) as implemented in DIVA v1.1 (Ronquist, 1996). DIVA is an event-based (vs. pattern-based) method, which uses a three-dimensional cost matrix to infer ancestral distributions, without the need of predefining general area relationships. In the simple underlying biogeographic model, speciation, which is assumed to occur mainly via vicariance, occurs at no cost, whereas dispersal and extinction are associated with costs. Thus, the method assigns hypothetical distributions to each of the internal nodes of a given topology with given distributions of the terminal taxa, while minimizing the costs (Ronquist, 1996). We designated eight unit areas as shown in Fig. 4.5, based on the biogeographic zones established by Martin (1972), Richard and Dewar (1991), Martin (1995), Ganzhorn et al. (2006), which were originally based on Humbert (1955). Each of the 14 terminal clades of our mtDNA haplotype phylogeny was scored according to its presence or absence in each of the eight geographical 
Table 4.1: Sampling localities. Site number as used in Fig. 4.5 locality name, number of individuals from the respective sampling site according to species, classification of sampling site according to biogeographic zones based on phytogeography adapted from Humbert (1955), Martin (1972), Richard and Dewar (1991), Martin (1995), Ganzhorn et al. (2006) and centers-of-endemism hypothesis as defined by Wilmé et al. (2006).

\begin{tabular}{|c|c|c|c|c|c|c|}
\hline site no. & locality & $\mathrm{N}_{\text {medius }}$ & $\mathrm{N}_{\text {major }}$ & $\mathrm{N}_{\text {crossleyi }}$ & $\begin{array}{l}\text { biogeographic } \\
\text { zone }\end{array}$ & $\begin{array}{l}\text { center of } \\
\text { endemism }\end{array}$ \\
\hline 1 & Manongarivo & - & - & 1 & $\mathrm{x} / \mathrm{SA}$ & 10 \\
\hline 2 & Ampasindava Bay & 1 & - & - & $\mathrm{x} / \mathrm{SA}$ & 10 \\
\hline 3 & Ambanja & 1 & - & 1 & $\mathrm{x} / \mathrm{SA}$ & 11 \\
\hline 4 & Ambato & 1 & - & - & $\mathrm{x} / \mathrm{SA}$ & 11 \\
\hline 5 & Foret de l'Ankarana & 1 & - & - & $\mathrm{x} / \mathrm{SA}$ & $?$ \\
\hline 6 & Montagne d'Ambre & - & - & 3 & $\mathrm{~N}$ & 1 \\
\hline 7 & Bekaraoka forest & 1 & - & - & $\mathrm{N}$ & 1 \\
\hline 8 & Iharana/Vohemar & - & - & 1 & $\mathrm{~N}$ & 1 \\
\hline 9 & Sambava & 4 & - & 9 & E1 & 2 \\
\hline 10 & Manantenina & - & - & 6 & E1 & 2 \\
\hline 11 & Maroantsetra2 & - & 1 & - & E1 & 2 \\
\hline 12 & Maroantsetra1 & - & 3 & - & E1 & $?$ \\
\hline 13 & Ile Ste. Marie & - & 1 & - & E1 & 2 \\
\hline 14 & Lake Alaotra, forest & - & - & 1 & $\mathrm{CH}$ & 2 \\
\hline 15 & Passumbé & - & 1 & - & E1 & 2 \\
\hline 16 & Tampolo & - & 3 & - & E1 & 2 \\
\hline 17 & Mahambo & - & 1 & - & E1 & 2 \\
\hline 18 & Toamasina/Tamatave & - & 1 & - & E1 & 2 \\
\hline 19 & Andasibe & - & - & 1 & E1 & 2 \\
\hline 20 & Mahanoro & - & 1 & - & E1 & $?$ \\
\hline 21 & Marolambo & - & 5 & - & $\mathrm{E} 2$ & $\mathrm{~B}$ \\
\hline 22 & Imerina, E. & - & 1 & - & $\mathrm{CH}$ & $?$ \\
\hline 23 & Ankazomivady & - & - & 3 & $\mathrm{CH}$ & $\mathrm{E}$ \\
\hline 24 & Ranomafana & - & - & 1 & E2 & 3 \\
\hline 25 & Andrambovato & - & 2 & 1 & E2 & 3 \\
\hline 26 & Ste. Luce & 7 & - & - & $\mathrm{E} 2$ & 5 \\
\hline 27 & Farafara & - & 4 & - & $\mathrm{E} 2$ & 5 \\
\hline 28 & Ivorona & - & 3 & - & E2 & 5 \\
\hline 29 & Mandena & 5 & 4 & - & E2 & 5 \\
\hline 30 & Manantantely & - & 3 & - & E2 & 5 \\
\hline 31 & Andohavondro & - & 2 & - & E2 & 5 \\
\hline 32 & Petriky & 1 & - & - & E2 & 5 \\
\hline 33 & Lavasoa & 4 & - & 7 & $\mathrm{E} 2$ & 5 \\
\hline 34 & $170 \mathrm{~km}$ East of Tulear & 2 & - & - & $\mathrm{W} 2$ & e6 \\
\hline 35 & Tabiky & 1 & - & - & $\mathrm{W} 2$ & $\mathrm{E}$ \\
\hline 36 & Morondava & 2 & - & - & $\mathrm{W} 2$ & 7 \\
\hline 37 & Kirindy & 7 & - & - & $\mathrm{W} 2$ & 7 \\
\hline 38 & Bemaraha & 2 & - & - & W1 & 8 \\
\hline 39 & Ampijoroa & 1 & - & 1 & NW & G \\
\hline
\end{tabular}




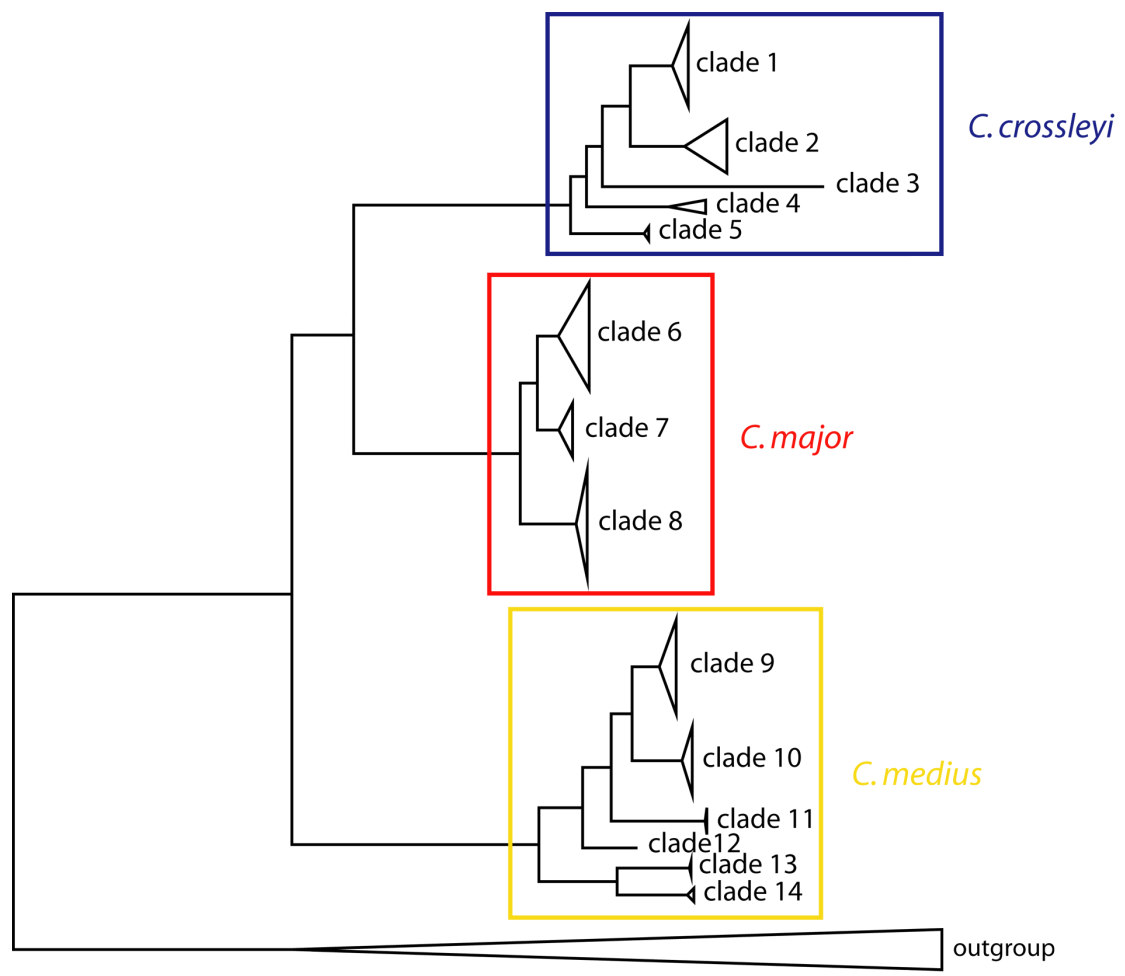

Figure 4.1: Simplified maximum likelihood phylogram of 113 Cheirogaleus individuals (62 haplotypes) based on $1824 \mathrm{bp}$ of mtDNA (cytb+cox2). Terminal taxa have been pooled to form 14 terminal clades. For a detailed version of this phylogram see chapter 3. The three species are marked by colored frames: $C$. medius=yellow, $C$. major $=$ red and $C$. crossley $i=$ blue.

areas (Fig. 4.5). Unconstrained optimization was carried out.

\subsubsection{Time divergence estimation}

Sequence data of five loci for 41 strepsirrhine species plus three outgroup species (human, chimpanzee and macaque) was included in the second part of this study dealing with time divergence estimates (see Appendix: Table C.1). Data from Microcebus, Mirza and Cheirogaleus individuals were added to a subset of taxa and loci taken from Horvath et al. (2008). An alignment was formed by concatenating two mitochondrial [cytochrome b (cytb), cytochrome oxidase II (cox2)] and three nuclear loci [adenosine receptor A3 exon 2 (adora3), alpha fibrinogen intron $4(f i b a)$, and von Willebrand Factor intron $11(v W F)]$. This alignment, amounting to $3712 \mathrm{bp}$, and an alignment consisting only of the two mitochondrial loci, amounting to $1824 \mathrm{bp}$, formed the basis of all time divergence estimates.

A Bayesian MCMC method, which employs a relaxed molecular clock approach (Drummond et al. 2006), as implemented in BEAST v1.4.6 (Drummond, 2007), was used to simultaneously estimate phylogeny and divergence times. A relaxed lognormal model of lineage variation and a Yule prior for branching rates was assumed. The alignment was partitioned according to loci. Models of nucleotide substitution and substitution rates were set separately for each 
Table 4.2: Settings of three BeAst analyses. For each analysis ('combined data', 'mtDNA' and 'unconstrained topology') the number of included loci and basepairs, the $95 \%$ confidence interval in mya of each calibration used, monophyly constraint settings and the number of replicates conducted are given.

\begin{tabular}{llll}
\hline & Combined data & MtDNA & Unconstrained topology \\
\hline \# loci (basepairs) & $5(3712)$ & $2(1824)$ & $5(3712)$ \\
Prior R & $73-90$ & - & - \\
Prior C1 & $38-42$ & $38-42$ & $38-42$ \\
Prior C2 & $5-7$ & $5-7$ & $5-7$ \\
Monophyly constraints & yes & yes & no \\
\# replicates & 3 & 2 & 2 \\
\hline
\end{tabular}

locus. Optimal nucleotide substitution models for each locus were chosen using the Akaike Information Criterion (AIC) as implemented in Modeltest 3.7 (Posada and Crandall, 1998). Substitution rates were calculated per locus based on the dated galago/loris divergence and used as priors for the mean of the branch rates (ucld.mean). No fossil-based calibrations are available for the Lemuriformes (e.g. Simons et al., 1995, Krause et al., 1997, Krause, 2003, de Wit, 2003). Therefore, calibrations based on combined fossil and molecular evidence from outside the Lemuriformes had to be included in our data set. We used the split between lorises and galagos, which has been timed at 38-42 mya by Seiffert et al. (2003) and the split between humans and chimpanzees, which has been dated at 5-7 mya according to Kumar et al. (2005). Furthermore, in one instance, it was necessary to constrain the age of the root of the tree. We used a mean of 81.5 mya for the least common ancestor of extant primates as estimated by Tavare et al. (2002). Instead of hardbounded calibration points, we used the published dates as a normal distribution prior for the respective node (in Fig. 4.2. C1, C2, R). For C1 (loris/galago) this translates into a normal distribution with a mean of 40 mya and a standard deviation of $1.215 \mathrm{ma}$, for $\mathrm{C} 2$ (human/chimpanzee) into a mean of 6.0 mya and a standard deviation of $0.62 \mathrm{ma}$ and for the root $(\mathrm{R})$ into a mean of 81.5 mya and a standard deviation of 5.0 my (95\% credibility interval: 73.28 - 89.72 mya). For all BEAST analyses, either 2 or 3 replicates (Table 4.2) were run for 25 million generations with tree and parameter sampling occurring every 2500 generations.

The adequacy of a $10 \%$ burnin and convergence of all parameters were assessed by visual inspection of the trace of the parameters across generations using the software TRACER v1.3 (Rambaut and Drummond, 2005). Subsequently, the sampling distributions of multiple independent replicates were combined using the software LOGCOMBINER v1.4.6 and then summarized and visualized using the software TreeAnnotator v1.4.6. Both programs are part of the BEAST package (Drummond, 2007).

Three different analyses were conducted (Table 4.2). The first analysis, referred to as 'combined data', included all five loci (3712 bp), all three calibrations (C1, C2 and R) and monophyly constraints on nodes C1-2, N1-6, N8, N14, the node encompassing all Lemuridae, $\mathrm{N} 4+$ Lepilemur, and N4+Lepilemur + Propithecus. Three independent replicates of this anal- 


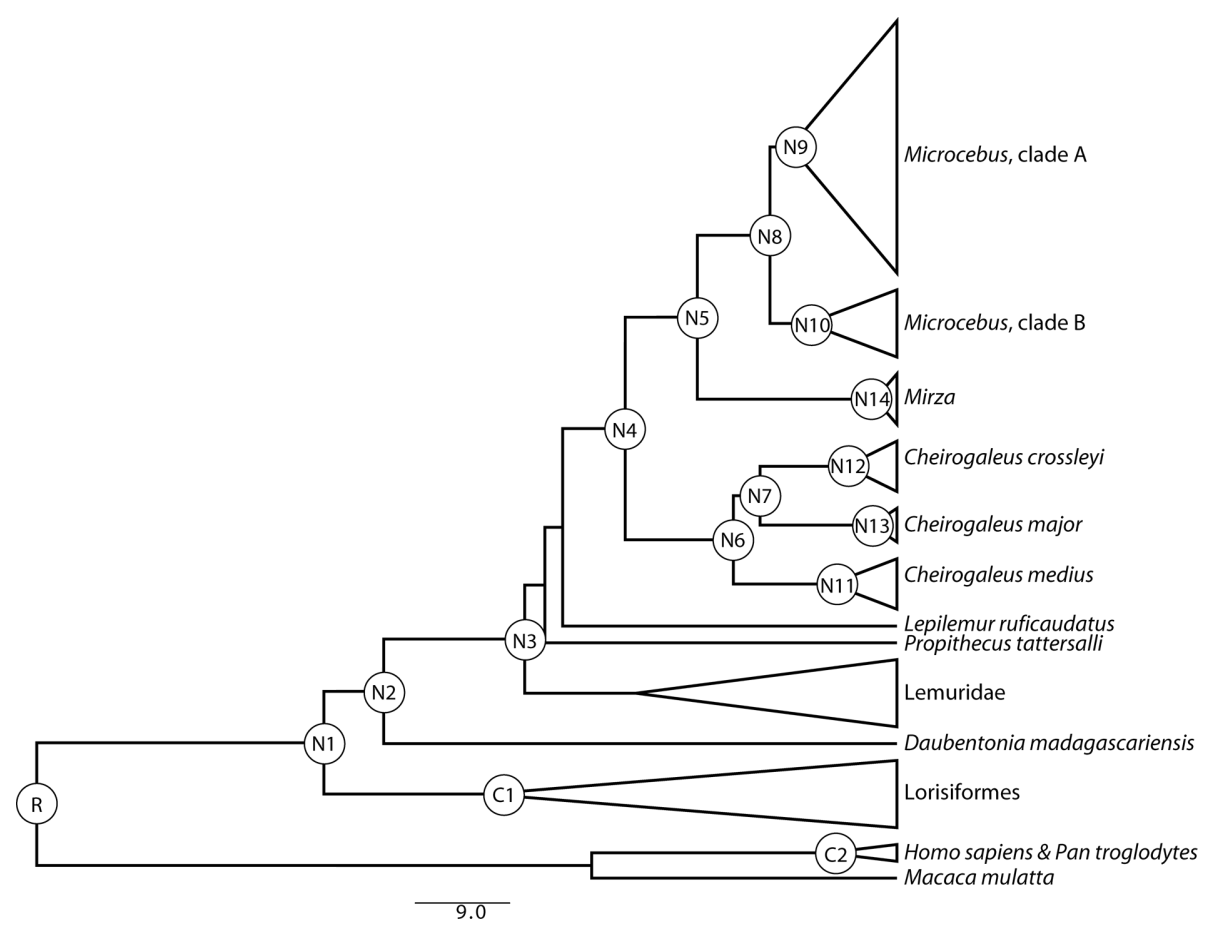

Figure 4.2: Simplified phylogenetic tree as estimated by BEAST. C1 and C2 are calibration nodes. $\mathrm{R}$ refers to the root, which was used as a calibration point in the 'combined data' analysis. N1-N14 refer to nodes for which divergence dates were estimated. The basic topology was defined a priori based on Horvath et al. (2008), for the 'combined data' and the 'mtDNA' analyses, by specification of monophyly constraints on certain nodes as described in subsection 4.2.2.

ysis were carried out. The second analysis, referred to as 'mtDNA', included only the two mtDNA loci (1824 bp), only calibrations C1 and C2 and all monophyly constraints as listed above. The third analysis, termed 'unconstrained topology', included all five loci (3712 bp), calibrations $\mathrm{C} 1$ and $\mathrm{C} 2$ and no monophyly constraints. Two independent replicates of the second and third analysis were conducted.

\subsection{Results}

\subsubsection{Concordance with 'centers-of-endemism' hypothesis}

The latest Cheirogaleus taxonomy accepts at least three species (see chapter 2 and 3), all of which are represented in this data set. The sampling sites, color-coded according to species, are depicted in Fig. 4.3, which also displays the centers of endemism (1-12) and retreat dispersion watersheds (A-J) according to Wilmé et al. (2006). Cheirogaleus medius individuals were found in seven different centers of endemism $(1,2,5,7,8,10,11)$ and in three retreatdispersion watersheds (e6, E, G). Cheirogaleus major individuals were present in three centers of endemism $(2,3,5)$ and in one retreat-dispersion watershed (B). Cheirogaleus crossleyi individuals were found in six centers of endemism $(1,2,3,5,10,11)$ and in two retreatdispersion watersheds $(\mathrm{E}, \mathrm{G})$. The three species were not only found in multiple centers 
of endemism and retreat-dispersion watersheds, but their presence was in many cases not exclusive.

In order to rule out that significant patterns at a level of higher resolution went undetected, we mapped the 14 terminal clades, defined through phylogenetic analyses of $1824 \mathrm{bp}$ of mtDNA (Fig. 4.1), onto the map of the centers of endemism and retreat-dispersion watersheds (Fig. 4.4). Clade 1 individuals are present in three centers of endemism $(2,10,11)$ in the northeast and northwest. Representatives of clade 2 are found in two centers of endemism $(1,2)$ in the northeast and east and in two retreat-dispersion watersheds (E, G). Clade 3, clade 4 and clade 5 individuals are found in one center of endemism in the southeast, east and northeast, respectively $(5,3,1)$. Individuals representing clade 6 are found in the east in one center of endemism (2) and at least one other zone, which due to inexact sampling locality information cannot be defined precisely. Clade 7 individuals are present in one center of endemism (3) and one retreat-dispersion watershed (B) in the east. Clade 8 individuals are present in one center of endemism (5) in the southeast. Individuals belonging to Clade 9 are present in two centers of endemism $(7,8)$ in the west and two retreat-dispersion watersheds (E, e6). Clade 10 individuals are found in one center of endemism (5) in the southeast. Representatives of clade 11 are found in the northwest in two centers of endemism $(10,11)$, while clade 12 is found in one retreat-dispersion watershed $(\mathrm{G})$. Clade 13 individuals are found in the northeast in one center of endemism (2). Representatives of clade 14 are found in the northeast in one center of endemism (1) and in at least one other zone in the north, which due to inexact sampling locality information cannot be defined precisely.

Most clades are found in multiple centers of endemism and most are not exclusive representatives of the respective zones. Clade 9 is the only exception: it is found exclusively in its zones, but spans two centers of endemism and two retreat-dispersion watersheds. No concordance between the phylogeny of Cheirogaleus individuals at the level of species or at a level of finer resolution (14 terminal clades) with the 'centers-of-endemism' hypothesis (Wilmé et al., 2006) can be found.

\subsubsection{Concordance with 'biogeographic' zones based on phytogeography}

The eight biogeographic zones based on phytogeography and the sampling sites are depicted in Fig. 4.5. A simplified version of the mtDNA phylogeny and the biogeographic zones that correspond to the 14 terminal clades are shown in Fig. 4.6A. Cheirogaleus medius individuals were found in all biogeographic zones, except the central highlands $(\mathrm{CH})$. Cheirogaleus major individuals were only present in the two eastern zones (E1, E2) and the central highlands $(\mathrm{CH})$. Cheirogaleus crossleyi was found to be present in all of the zones except in the western zones (W1, W2).

Only the two western zones (W1, W2) were exclusively inhabited by only one species, $C$. medius. This species, however, was not confined to these two zones, but present in all other zones except the central highlands $(\mathrm{CH})$. In all other zones (apart from the discussed W1 and W2) multiple species were found to be present. At the species level, no concordance could be 
detected between the biogeographic zones and Cheirogaleus distributions.

At a higher level of resolution (14 terminal clades) representatives of nine clades were only present in one single zone (clades 3-5, 7, 8, 10-13). However, the clades were not exclusive to the zones. A further four clades were each found to be present in two zones (clades 1, 6, $9,14)$. One clade was found to be present in four zones (clade 2). Concordance among the biogeographic zones and Cheirogaleus distributions can neither be found on the specific level, nor on a level of higher resolution.

Based on the species composition of lemur communities as known in 1999, the biogeographic zones are linked as shown in Fig. 4.6B (Ganzhorn et al., 2006). In general a 'western clade' consisting of zones W1, W2 and NW is contrasted by an 'eastern clade' consisting of zones $\mathrm{E} 1, \mathrm{E} 2, \mathrm{~N}$ and $\mathrm{x} / \mathrm{SA}$. Cheirogaleus distributional data do not confirm this pattern (Figures $4.6 \mathrm{~A} \& \mathrm{~B})$.

\subsubsection{Ancestral area reconstruction}

Ancestral area reconstruction found an exact solution, with 36 equally optimal reconstructions, all of which require ten dispersal events. One optimal reconstruction is depicted in Fig. 4.6A. The most recent common ancestor of C. crossleyi was accordingly present in zones $\mathrm{N}$ and E2, which are disjunct. Cheirogaleus major was found to have originated in zone E2 and C. medius was only found not to have originated in $\mathrm{E} 2$ or $\mathrm{CH}$. It should be noted that even though DIVA has a tendency for imprecise assignment of ancestral distribution towards the root node, the ancestral distribution of C. major and C. crossleyi, respectively, was unequivocally recovered. The ancestral distribution of $C$. major plus $C$. crossleyi should not be over interpreted, since statistical support for this node is rather low (see chapter 3, Fig. 3.2).

\subsubsection{Age estimates of cheirogaleid divergences}

The complete alignment consisting of five loci (cytb, cox2, adora3, fiba and $v W F$ ) contained six missing sequences: three cox2 sequences (Galago moholi, Nycticebus pygmaeus and Otolemur garnetti) plus three fiba sequences (Galago moholi, Nycticebus coucang and Loris tardigradus).

Table 4.3: Nucleotide substitution models and substitution rates per locus. The best fit model according to AIC and the model used in BEAST analyses are given. The nucleotide substitution rates were calculated for the galago/loris split. For the cox2 locus the respective sequence data was not available and the galago/loris rate therefore estimated using rates from the human/chimpanzee split.

\begin{tabular}{llll}
\hline & Model & & Rate \\
& AIC & BEAST & \\
\hline cytb & $\mathrm{TVM}+\mathrm{I}+\Gamma$ & $\mathrm{GTR}+\mathrm{I}+\Gamma$ & 0.0024 \\
cox2 & $\mathrm{HKY}+\mathrm{I}+\Gamma$ & $\mathrm{HKY}+\mathrm{I}+\Gamma$ & 0.0020 \\
adora3 & $\mathrm{GTR}+\Gamma$ & $\mathrm{GTR}+\Gamma$ & 0.0006 \\
fiba & $\mathrm{TVM}+\Gamma$ & $\mathrm{GTR}+\Gamma$ & 0.0009 \\
$v W F$ & $\mathrm{TVM}+\Gamma$ & $\mathrm{GTR}+\Gamma$ & 0.0009 \\
\hline
\end{tabular}


Furthermore, several sequences contained missing data in the fiba and $v W F$ fragments, ranging from a few basepairs to around $250 \mathrm{bp}$. No indels were present in the adora3, cytb and cox2 fragments, but multiple indels characterized the fiba and $v W F$ fragments. The complete data set contained 3712 bp of aligned sequence, of which 1888 characters were variable and 1522 were parsimony informative. When the nuclear loci were excluded, the mtDNA alignment contained 1824 bp of aligned sequence, of which 996 characters were variable and 874 parsimony informative.

The $c y t b$, fiba and $v W F$ loci were each found to best fit a transversion model (TVM) according to AIC, but were analyzed under a general time reversible (GTR) model. The nuclear loci were best fit to a model with gamma distributed rate heterogeneity $(\Gamma)$, whereas the $c y t b$ locus was best fit to a model with both gamma distributed rate heterogeneity $(\Gamma)$ and a proportion of invariant sites (I). The adora3 locus was found to best fit a GTR $+\Gamma$ model, whereas the cox2 locus was found to best fit a Hasegawa-Kishino-Yano (HKY) $+\Gamma+\mathrm{I}$ model (Table 4.3). Substitution rates, calculated based on the dated galago/loris divergence, ranged from 0.002 for the cox2 locus to 0.0009 for the fiba and $v W F$ loci. All rates are given in Table 4.3 .

The estimation of a strepshirrine phylogeny was not the main aim of this study and for two of the three BEAST analyses the topology was fixed a priori according to analyses of 18 nuclear loci by Horvath et al. (2008). Instead, we focused on divergence date estimates within the Cheirogaleidae, but age estimates for deeper nodes are also given in Table 4.4 for comparative purposes.

Age estimates yielded by the 'combined data' and the 'mtDNA' analyses were very similar. Only the $95 \%$ credibility interval for the root is larger in the 'mtDNA' than in the 'combined data' analysis, due to no calibration prior having been enforced on the root in this analysis. The 'unconstrained topology' analysis resulted in an altered topology, with Daubentonia madagascariensis placed basal to all other strepsirrhines and Lepilemur ruficaudatus and Propithecus tattersalli emerging as sister taxa. The age estimates for this analysis are slightly older among the deeper nodes. For the nodes of interest, within the Cheirogaleidae, the estimates do not differ by more than 1 ma and the $95 \%$ credibility intervals largely overlap. Results of the 'combined data' analysis are shown in Fig. 4.7 and Table 4.4 and only age estimates from this analysis will be discussed in detail. The first divergence within the genus Cheirogaleus is estimated at 15.5 mya and divergence within species at 6.2 mya for C. medius, 4.8 mya for C. crossleyi and 2.1 mya for C. major. 


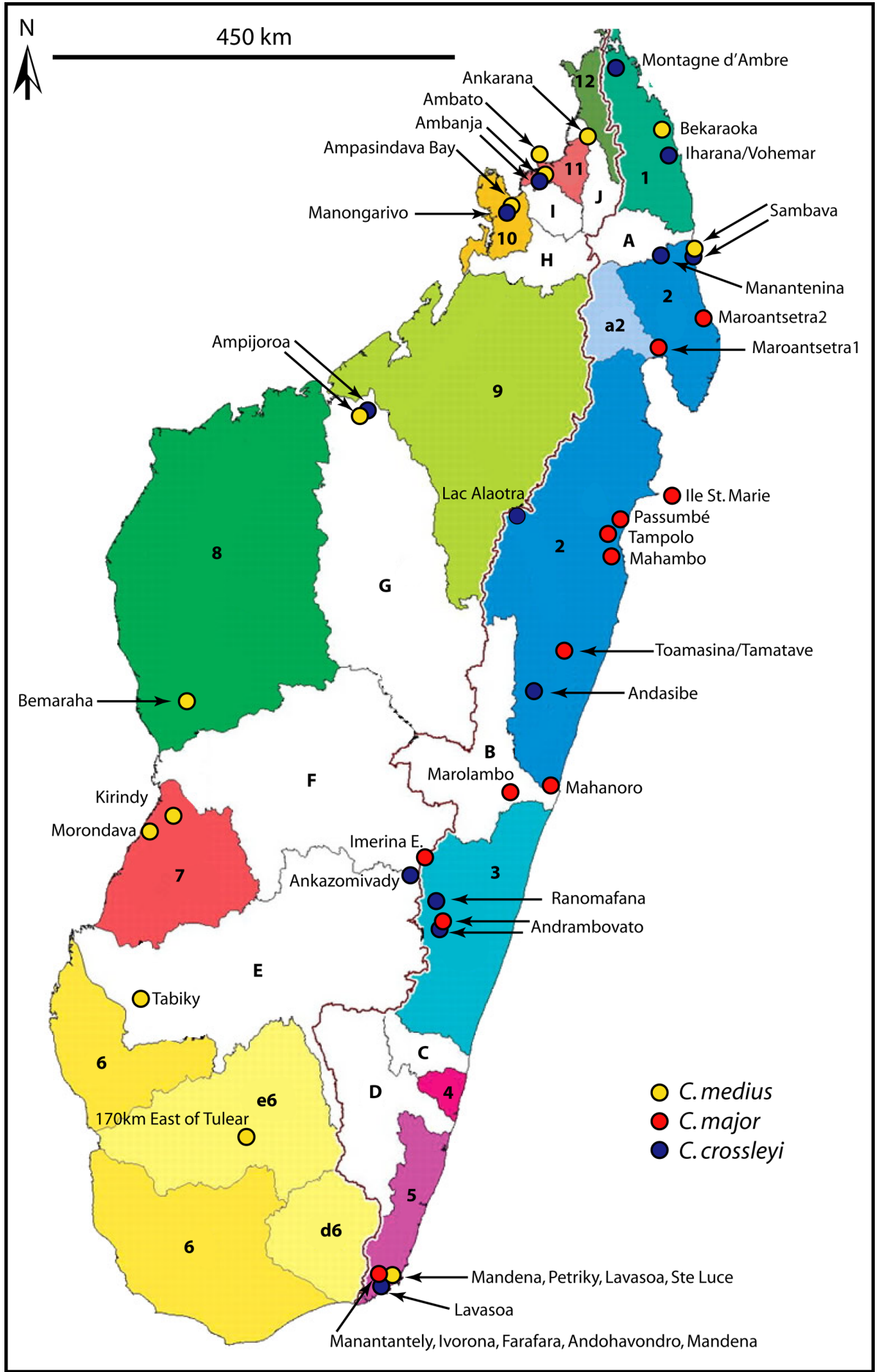

Figure 4.3: Map of Madagascar showing the sampling sites and the centers of endemism and retreat-dispersion watersheds according to Wilmé et al. (2006). The sampling sites are color-coded according to species as assessed in chapter 2 and 3, yellow $=C$. medius, red $=C$. major and blue $=C$. crossleyi. The sampling sites in the southeast (Fort Dauphin region) are pooled according to species. For a detailed description of these sites refer to Hapke et al. (2005). Map modified after Wilmé et al. (2006). 


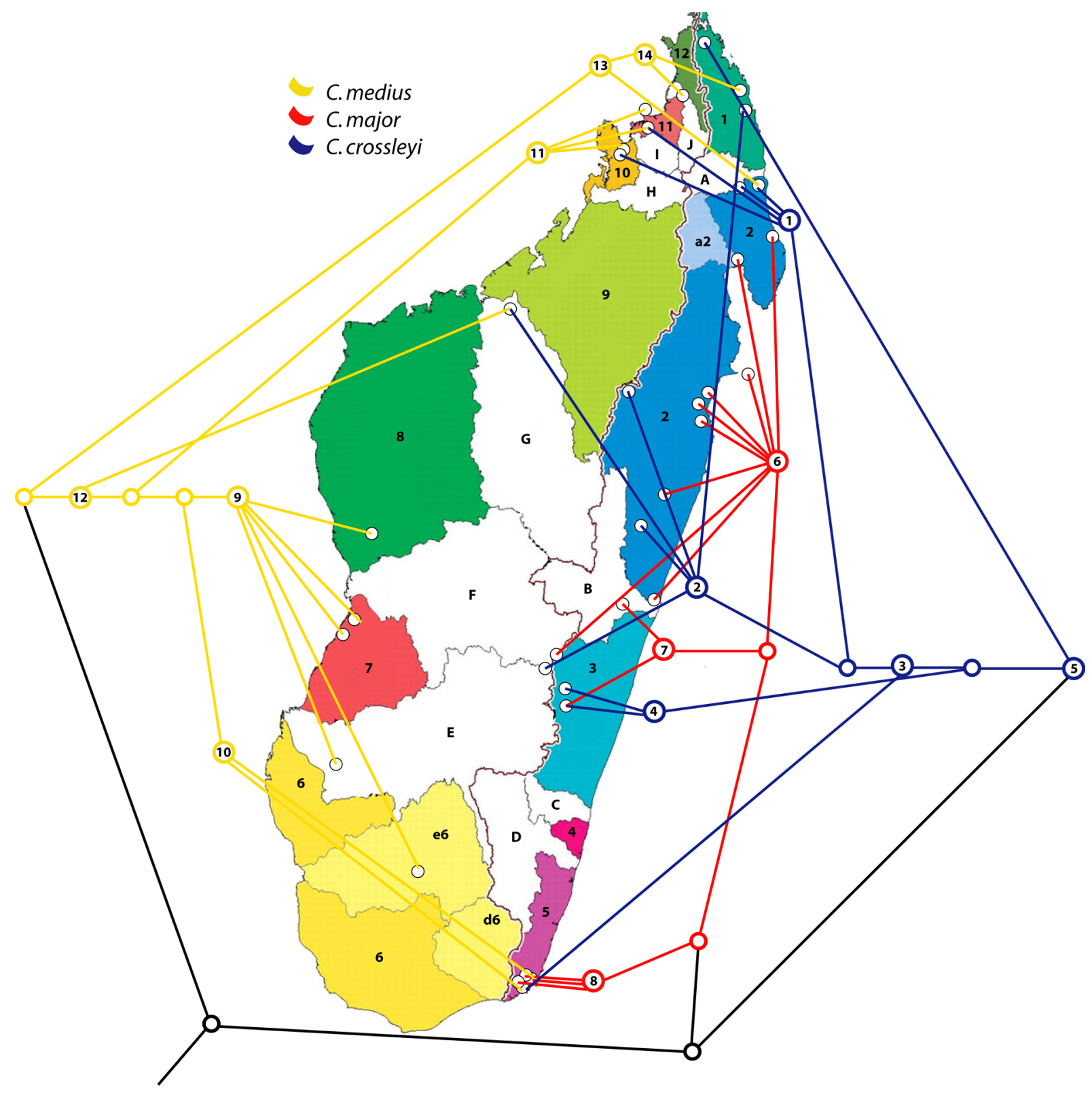

Figure 4.4: Simplified phylogeny as shown in Fig. 4.1 superimposed onto a map of Madagascar showing the sampling sites and the centers of endemism and retreat-dispersion watersheds according to Wilmé et al. (2006). Clades representing the different species are color-coded: $C$. medius $=$ yellow, $C$. major $=$ red and $C$. crossleyi=blue and the clade number as given in Fig. 4.1 is denoted. The sampling sites in the southeast (Fort Dauphin region) are pooled according to geographic location; see Fig. 4.5. For a detailed description of these sites refer to Hapke et al. (2005). Map modified after Wilmé et al. (2006). 


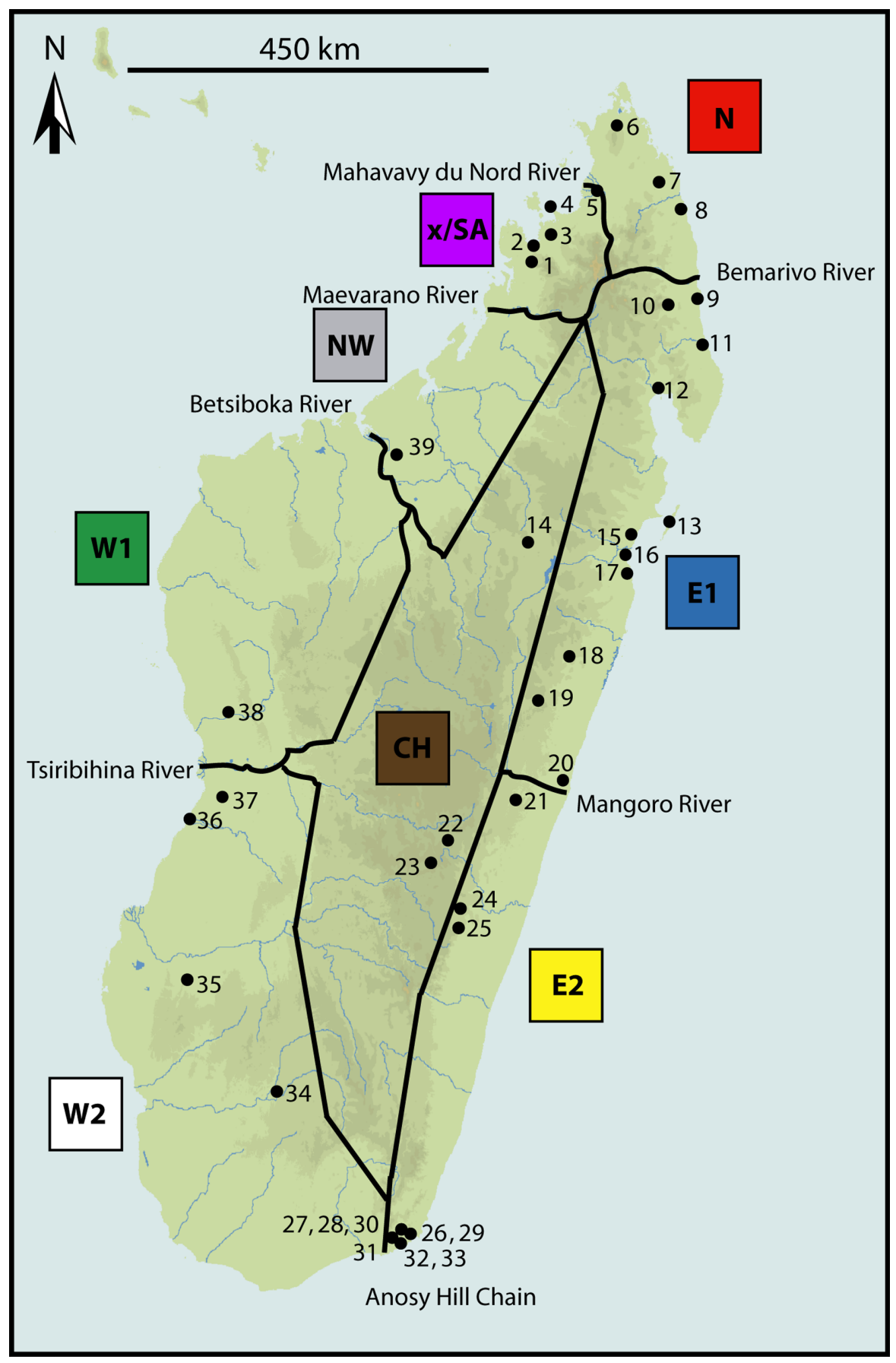

Figure 4.5: Map of Madagascar showing the 39 sampling sites and the eight biogeographic zones (N, E1, E2, W2, W1, NW, x/SA, CH) adapted from Martin (1972), Richard and Dewar (1991), Martin (1995), Ganzhorn et al. (2006). Limits of the biogeographic zones, excluding the central highlands $(\mathrm{CH})$, are defined through major rivers: Bemarivo, Mangoro, Tsiribihina, Betsiboka, Maevarano and Mahavavy du Nord rivers and a mountain chain: the Anosy hill chain. The limits of the biogeographic zones towards the inland $(\mathrm{CH})$ are defined mainly through elevation (800-1000m). The biogeographic zones are color-coded as indicated by the colored background of the label. The sampling sites are labeled by locality number as denoted in Table 4.1 . 


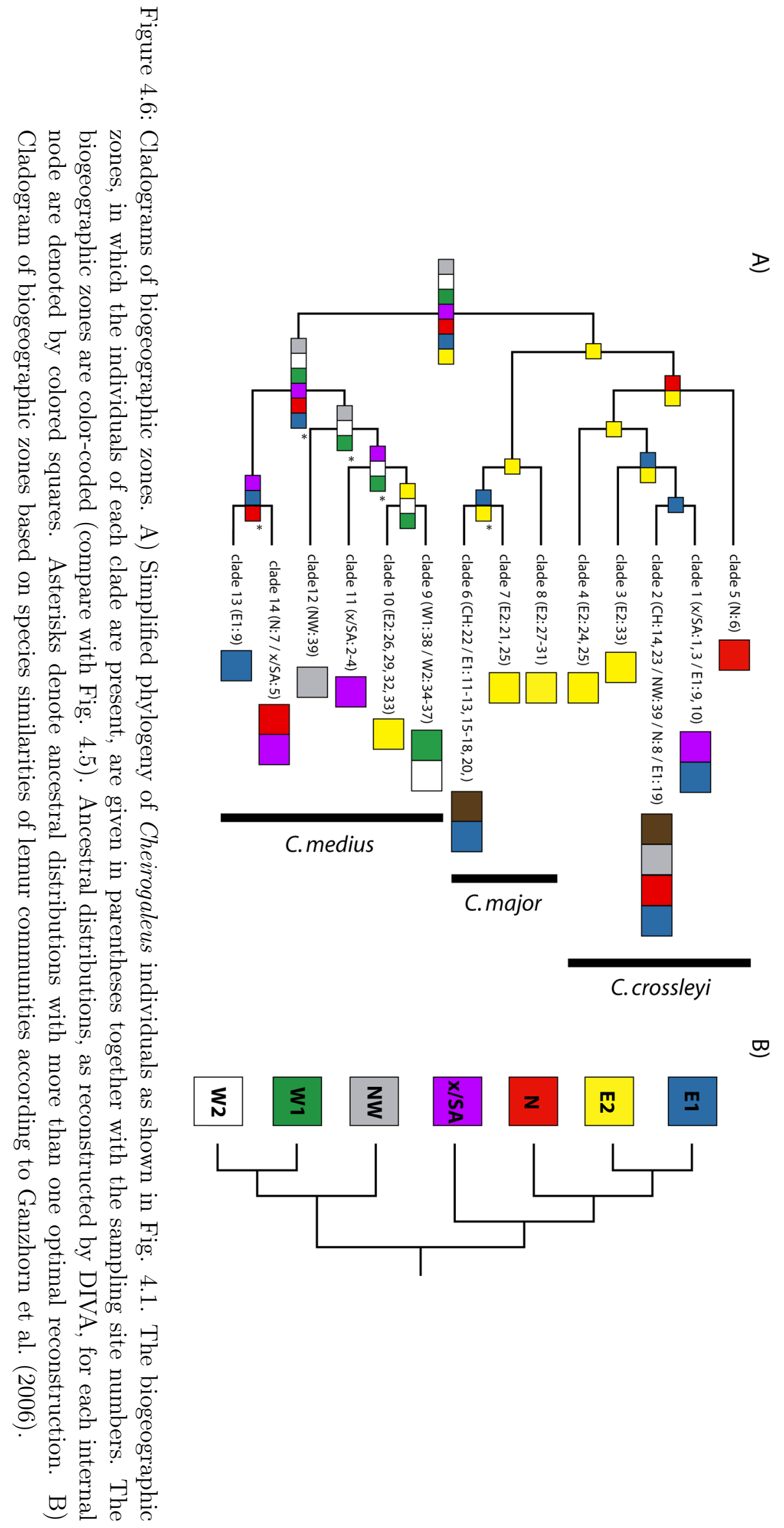




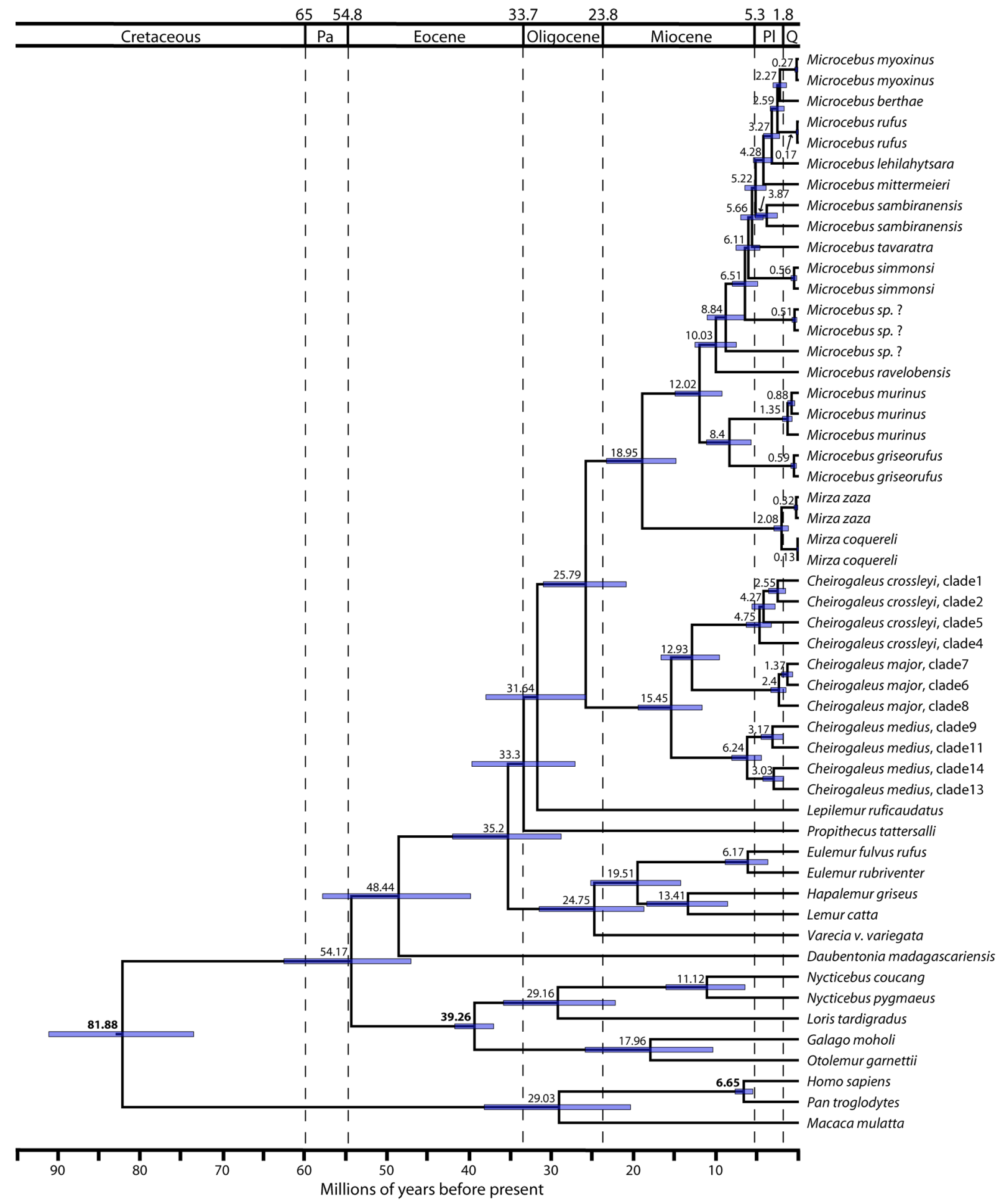

Figure 4.7: Ultrametric tree with divergence age estimates resulting from the combined posterior distribution of the three replicates of the 'combined data' BEAST analysis. The mean age estimate for each node is given in millions of years, with the respective $95 \%$ credibility intervals indicated by the blue bars. The means in bold indicate nodes used as calibrations. A geological time scale is given at the top: $\mathrm{Pa}=$ Paleocene, $\mathrm{Pl}=$ Pliocene and $\mathrm{Q}=$ Quaternary. Full details of age estimates of all BeAst analyses are presented in Table 4.4. Cheirogaleus individuals are assigned to clades as defined in Fig. 4.1. 


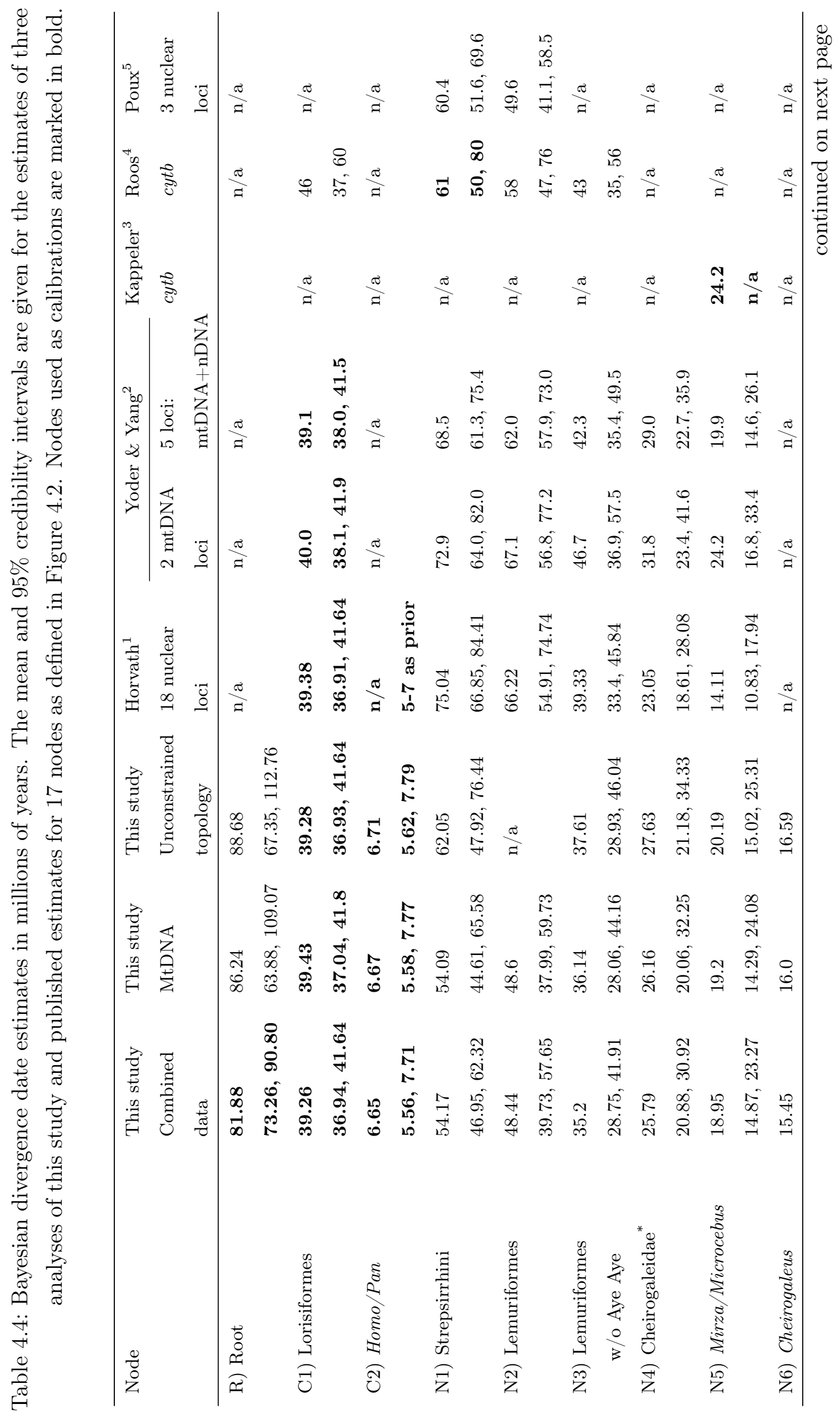




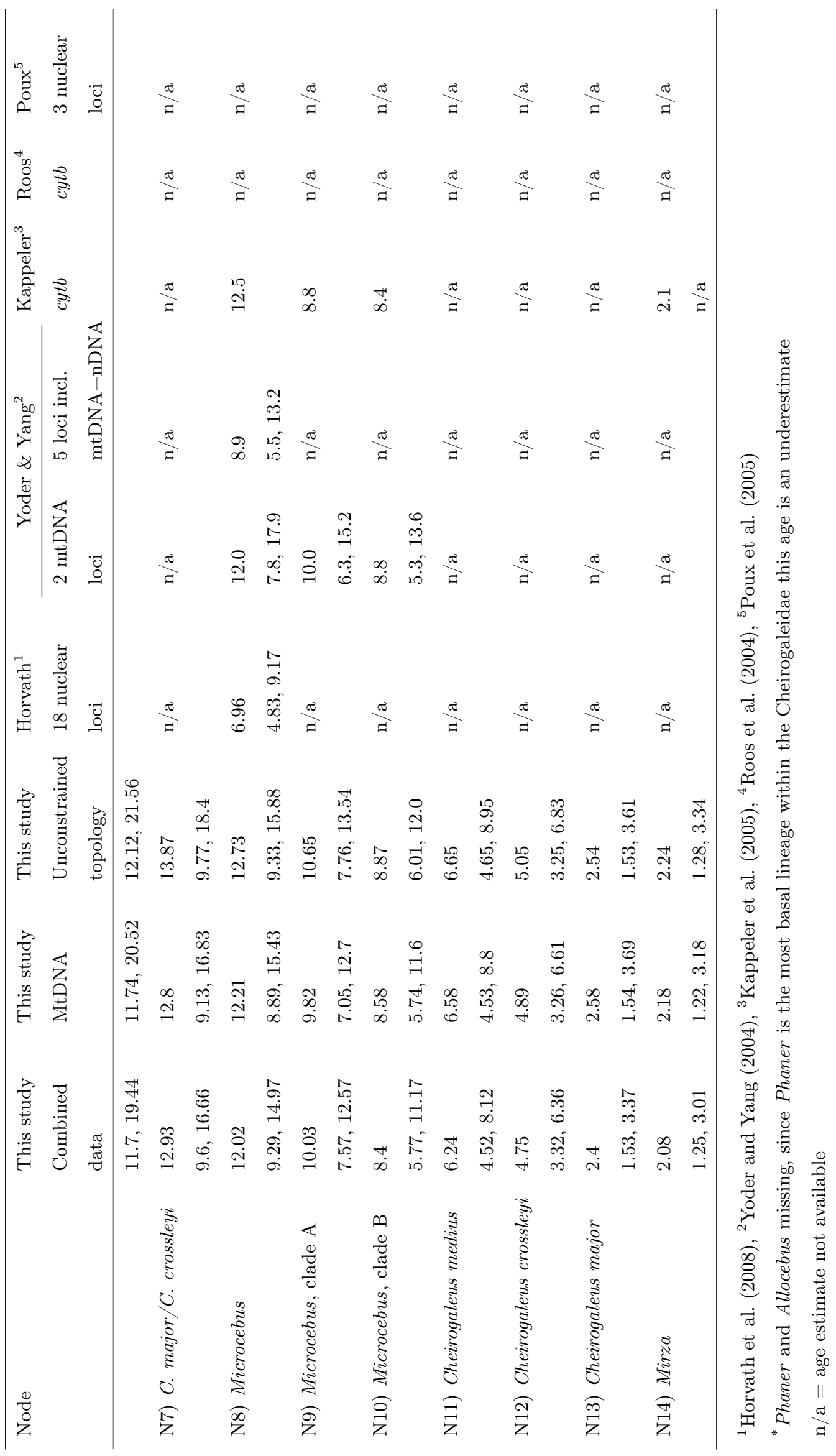




\subsection{Discussion}

\subsubsection{Concordance with published biogeographic hypotheses}

The 'centers-of-endemism' hypothesis predicts twelve centers of endemism, each potentially harboring a distinct species (Wilmé et al., 2006). Our samples only stem from eight centers of endemism; we have no data from the centers of endemism 4, 6, 9 and 12. This model would therefore predict a maximum of eight distinct species within our sample. At the level of species, as assessed through our genetic and morphometric analyses, we only found a total of three species and these are not exclusive to any single or combination of centers of endemism. All three species, C. medius, C. major and C. crossleyi, for example are present in centers of endemism 2 and 5. When inspecting a higher level of resolution, in form of mtDNA clades, no clear pattern following the centers of endemism emerges (see Fig. 4.4). Members of one clade are present in multiple, at times disjunct, centers of endemism and multiple clades are present in one center of endemism. Possibly our sampling scheme is too coarse to be able to recover distributional patterns at the level this model requires.

The biogeographic hypothesis based on phytogeography, which predicts a maximum of eight species, is also not concordant with our hypothesis at the level of species. Cheirogaleus medius is found in all zones except the central highlands. Cheirogaleus crossleyi is found in all zones except W1 and W2 and C. major is only present in zones E1, E2 and the central highlands. Especially in the case of $C$. medius this description of its distribution is misleading, since this species is not found along most of the east coast. It is merely present in the extreme south of $\mathrm{E} 2$ and the extreme north of E1. A clear pattern is also not discernible at the level of mtDNA clades (see Fig. 4.6).

\section{Rivers as barriers}

Rivers as barriers play a major role in both models (Martin, 1972, 1995, Wilmé et al., 2006). They have been discussed as barriers in regard to lemur dispersal in detail (Pastorini et al. 2003. Goodman and Ganzhorn, 2004a, Ganzhorn et al., 2006) and they have been attributed a major role in recent phylogeographic assessments of lemur diversity (Craul et al., 2007, Olivieri et al., 2007). In both models the following rivers are reported to represent a barrier separating species or subspecies of lemurs: Mahavavy du Nord, Bemarivo, Mangoro, Tsiribihina, Betsiboka and Maevarano (Fig. 4.5). In the following we will discuss each river's potential as a barrier to dispersal by examining the distribution of our mtDNA clades and integrating this with published data for other lemur taxa.

The Mahavavy du Nord could represent a barrier between clade 11 and clade 14, since the exact locality of the sample from Ankarana (sampling site 5) is not known. Recent diversity assessments of sportive lemurs (genus Lepilemur) and mouse lemurs (genus Microcebus) in the northwest have shown that this river acts a barrier between species in these genera (Craul et al., 2007, Olivieri et al., 2007).

The Bemarivo river could act as a barrier between clades 1 and 5, as well as between clades 
13 and 14, but individuals from clade 2 are found both north and south of the Bemarivo. However, the individual recorded to be from Iharana/Vohemar (sampling site 8), north of the Bemarivo, is a museum specimen (Hapke et al., 2005), for which sampling localities are often questionable. Goodman and Ganzhorn (2004a) provide an assessment of which species distributions support the hypothesis that certain rivers form barriers between lemur taxa. For the Bemarivo river they list three Eulemur taxa, and Indri indri of which only one taxon's distribution supports the hypothesis that the Bemarivo acts as a dispersal barrier.

The Mangoro river does not separate clade 2 from clade 4, but a separation of clade 6 and clade 7 by this river could be considered, because the only individual violating this separation is again a museum sample (sampling site 22, Imerina, E.; see chapter 3). For the Mangoro river Goodman and Ganzhorn (2004a) list five lemur taxa (two Eulemur subspecies, Indri indri and two Propithecus subspecies), three of which support the hypothesis of the Mangoro being a dispersal barrier.

The Tsiribihina river does not seem to pose a barrier within C. medius, since individuals from north and south of this river are found in clade 9. Pastorini et al. (2003) discuss the importance of this river as a barrier and conclude that the river does not serve as a barrier between Propithecus subspecies, but that genetic differentiation between Eulemur taxa north and south of the river are pronounced enough to warrant subspecific differentiation. The authors furthermore postulate that the Tsiribihina or the Betsiboka river might serve as a genetic barrier in C. medius. A taxonomic revision of the genus Lepilemur by Andriaholinirina et al. (2006) includes Lepilemur ruficaudatus samples from north and south of the Tsiribihina river, which in a mtDNA phylogeny show distinct differentiation. They conclude that further lines of corroborative evidence, such as nuclear DNA, are needed to warrant a species level differentiation. In contrast to Pastorini et al. (2003), Ganzhorn et al. (2006) list the Tsiribihina as separating the Propithecus subspecies that Pastorini et al. (2003) deemed as genetically too undifferentiated to warrant subspecific status. This, together with the Lepilemur ruficaudatus example, demonstrates how different taxonomies lead to starkly different conclusions in biogeographical analyses.

The Betsiboka and Maeverano rivers could represent barriers, but our sampling in this area is too scant to draw reliable conclusions. Both rivers play an important role in separating Lepilemur and Microcebus species; although further differentiation at the specific level between these rivers is found for both genera (Craul et al., 2007, Olivieri et al., 2007). The Betsiboka has been found to separate Eulemur fulvus subspecies and Propithecus verrauxi subspecies, but on the other hand does not seem to serve as a boundary for Eulemur mongoz populations (Pastorini et al., 2003, Ganzhorn et al., 2006).

As demonstrated by this short review of literature combined with our data, no generally applicable rule seems to be deducible for these eight rivers. 


\section{Rivers as corridors}

Multiple authors have pointed out that other qualities of rivers, than their width have to be taken into account when assessing their potential as dispersal barriers. The "centers-ofendemism' hypothesis is based on the idea that forests along rivers originating at high altitudes may have served as refugia during more arid conditions in the Pleistocene. Furthermore, rivers with sources at lower elevations, if they did not dry out completely, would have constituted isolated habitats, therefore driving vicariant events. Since headwaters of a number of the rivers originating at high altitudes are within close distance of each other, some of them draining eastwards and some westwards, dispersal in an eastern-western direction, and viceversa, would have been facilitated (Wilmé et al. 2006 ). Moreover, it has to be taken into account that species with a distribution above the elevation of the source of a river are able to circumvent that watercourse (Ganzhorn et al., 2006, Goodman and Ganzhorn, 2004a).

An eastern-western connectivity has been pointed out for a number of genera: Eulemur (e.g. Richard and Dewar, 1991, Martin, 1995, Ganzhorn et al., 2006), Propithecus (e.g. Ganzhorn et al. 2006), Microcebus (Yoder et al., 2000) and Cheirogaleus (Thalmann and Rakotoarison, 1994, Thalmann, 2000, Ausilio and Raveloanrinoro, 1998). Martin (1995) noted that it seemed likely that zones NW and N, as well as zones E1 and x/SA, are more similar to each other in lemur distribution patterns, than to their neighboring zone. This can be supported by our data. Cheirogaleus crossleyi individuals from clade 1 are found in E1 and $\mathrm{x} / \mathrm{SA}$ and clade 2 individuals are found in $\mathrm{N}$ and $\mathrm{NW}$ (plus in $\mathrm{E} 1$ and $\mathrm{CH}$ ), but not in $\mathrm{x} / \mathrm{SA}$.

All of these considerations become obsolete in explaining extant species distributions, if the temporal aspect is ignored (Donoghue and Moore, 2003). Tattersall (1982) pointed out that the biogeographic model based on phytogeography implies that climatic conditions in Madagascar were stable over a long period of time. This implicit assumption has been refuted by numerous authorities (e.g. Tattersall, 1982, Richard and Dewar, 1991, Burney, 1997, Burney et al., 2004, Goodman and Ganzhorn, 2004b). It is therefore crucial to have an understanding of the timeline on which lemur radiations took place.

\subsubsection{Time divergence estimates}

We estimated the divergence between the Lorisiformes and Lemuriformes at 54.2 mya and the first divergence within the Lemuriformes at 48.4 mya (Table 4.4). These estimated ages are substantially younger than previously published estimates by Horvath et al. (2008) and Yoder and Yang (2004). Their analyses arrived at mean estimates ranging from 69 to 75 mya for the first strepsirrhine divergence and at 62 to 67 mya for the first lemuriform divergence. For both nodes the $95 \%$ credibility intervals of our analysis and the published analyses do not or only barely overlap. Published estimates for these nodes by Roos et al. (2004) and Poux et al. (2005) are closer to our estimates with the $95 \%$ credibility intervals largely overlapping and mean estimates, especially for the first lemuriform divergence from Poux et al. (2005) being congruent with our estimate.

From the first divergence within the Cheirogaleidae towards the most recent divergences 
our estimates are essentially congruent with those published by Yoder and Yang (2004) and Kappeler et al. (2005). We estimated the divergence between Mirza and Microcebus to have occurred at 19.0 mya, whereas Yoder and Yang (2004) estimated 24.2 mya (mtDNA) and 19.9 mya (combined mtDNA and nDNA). Kappeler et al. (2005) used the mtDNA estimate for the Microcebus/Mirza divergence as a calibration and similar results for these two data sets within the Cheirogaleidae are therefore not surprising. Our estimates for the first divergence within the genus Microcebus at 12.0 mya, the first divergence within Microcebus clade A and $\mathrm{B}$ at 10.0 mya and 8.4 mya, respectively and the divergence between Mirza coquereli and Mirza zaza at 2.1 mya are congruent with the estimates based on mtDNA by Yoder and Yang (2004) and Kappeler et al. (2005). The 95\% credibility intervals of our analysis are nested within those of the estimates by Yoder and Yang (2004).

The most recently published estimates by Horvath et al. (2008) are based on 18 nuclear loci, comprising 14,500 bp of aligned sequence and represent the most robust age estimate for Madagascar's lemurs available. Our data are directly comparable to the analysis of Horvath et al. (2008), since we used the same calibrations and our nuclear data represent a subset of their data. However, it has to be noted that our 'combined data' analysis contains two mtDNA loci, which Horvath et al. (2008) excluded from their analyses, mainly due to node support diminishing when mtDNA was added to the nuclear data. Our age estimates differ from those of Horvath et al. (2008) in that our deep nodes are considerably younger and the more recent divergences are much older than the respective estimates from Horvath et al. (2008). Our divergence estimate between the Lorisiformes and Lemuriformes lies at 54.2 mya, whereas Horvath et al. (2008) estimate this divergence at 75.0 mya. The $95 \%$ credibility intervals for this node do not overlap. The estimates for the first divergence within the Cheirogaleidae (in both cases excluding Phaner and Allocebus) are much closer, with our estimate at 25.8 mya and Horvath et al. (2008) at 23.1 mya; the 95\% credibility intervals largely overlap. The first divergence within the genus Microcebus is estimated by Horvath et al. (2008) at 7.0 mya, whereas our estimate is 12.0 mya; the $95 \%$ credibility intervals do not overlap for this node. This discrepancy between our estimates and those by Horvath et al. (2008) could possibly be explained by our inclusion of mtDNA loci. This would be supported by the concordance of our estimates with those of Yoder and Yang (2004), in which mtDNA was also included. However, including mtDNA does not explain the rather young estimates for the lemuriform/ lorisiform divergence and the initial lemuriform radiation.

The first divergence within the genus Cheirogaleus is estimated at 15.5 mya and divergence within species at 6.2 mya for C. medius, 4.8 mya for C. crossleyi and 2.1 mya for C. major. Should a larger data basis, as employed by Horvath et al. (2008), prove that our estimates are biased towards deeper nodes being too young and recent nodes being too old, then our age estimates for the cheirogaleids would be overestimates. Even though methods for estimating clade ages are getting more sophisticated, they are not infallible and the age estimates have to be viewed as approximations (e.g. Yoder and Nowak, 2006). 


\subsubsection{Age of cheirogaleid species in the context of biogeographic hypotheses}

According to our estimates, species level divergences within the dwarf lemurs took place between 1.5 and 8.1 mya (95\% credibility intervals). This indicates that the origins of dwarf lemur species largely predate the Quaternary. Since, the 'centers-of-endemism' hypothesis is based on the assumption that Quaternary shifts of ambient temperature led to the processes driving speciation (Wilmé et al., 2006), our age estimates do not support this hypothesis.

No explicit temporal information is provided by the authors of the biogeographic zones based on phytogeography, but Wells (2003) argues that the different biomes correspond well to regional climatic variations (rainfall and seasonality), which in turn are the result of topography, plate tectonics and regional patterns of air and water circulation. Since the age of these factors can be estimated, an idea of the antiquity of the biomes, in turn, can be deduced.

He concludes that the xeric spiny bush is the oldest formation, which arose during the northward drift of Madagascar through the belt of high-pressure deserts during the late Cretaceous to the Eocene. As Madagascar emerged north of this arid belt and into the moist trade winds, this biome contracted into the southwestern corner of the island and the western deciduous forests would have formed. The humid eastern forests receive orographic rainfall from the trade winds and the warm waters off the east coast. The currents responsible for the warm waters probably did not exist in their contemporary form until the landmasses of India, Australia and Antarctica had moved out of the way in the Late Eocene or the Oligocene. Analyses of sediment compositions also support the onset of very warm and wet conditions along the east coast in the Oligocene (Wells and Andriamihaja, 1993). The 'Sambirano' humid forest $(\mathrm{x} / \mathrm{SA})$ biome is linked to the Indian monsoons, which in turn are coincident with the uplift of the Tibetan plateau. The monsoons are estimated to have started around 8 mya and to have steadily intensified since (e.g. Molnar et al., 1993). This biome would have been the most recently established formation, except for the central grasslands.

According to Wells' (2003) model, which he himself termed a 'working hypothesis', all extant biomes were present at the time of species level diversification in the dwarf lemurs. The oldest and most basal of the three dwarf lemur species, C. medius, in the only species found in the zone W2, which includes the oldest biome. Ancestral area reconstruction did not yield a clear result regarding the ancestral distribution of this species. The most recent common ancestor of C. crossleyi plus C. major was recovered to have been present in E2. The divergence between these two lineages is estimated at 9.6 to 16.66 mya (95\% credibility intervals), at which time the humid eastern forests would already have existed. The reconstruction of ancestral distributions in this case should be regarded with caution, since a potential sampling bias can easily influence the results.

Species level divergences within dwarf lemurs are of a similar age to species level divergences within mouse lemurs. The origins of mouse lemur species were estimated to lie between 1.5 and 12.8 mya (95\% credibility intervals); the same considerations regarding the models explaining speciation, as outlined above, thus apply for mouse lemurs. 


\subsection{Conclusion}

Neither the model of biogeographic zones based on phytogeography, nor the 'centers-ofendemism' hypothesis is concordant with, or explains the contemporary distribution of the currently recognized species of dwarf lemurs. This is true for the specific level, as well as at a higher level of resolution when examining mtDNA clades. The age estimates for dwarf lemur species range from 1.5 to 8.1 mya (95\% credibility intervals) and are comparable to estimates for mouse lemur species at 1.5 to 12.8 mya (95\% credibility intervals) and thus species level divergences in both genera largely predate the Quaternary. This indicates that models, such as the centers-of-endemism hypothesis, which assume that climatic shifts in the Quaternary were the driving force of speciation, do not apply.

The distributional data presented here are not exhaustive. Numerous authors have mentioned additional sites where dwarf lemurs are present (Rasolofoson et al., 2007, Thalmann and Rakotoarison, 1994, Thalmann, 2000, Ausilio and Raveloanrinoro, 1998, Mittermeier et al. 1994 and Laurie Godfrey/Marina Blanco, personal communication), which we were not able to include in this study. Morphometric and genetic data from the afore mentioned populations is needed in order to assess their species identity, so that these populations can be integrated into future phylogeographic analyses.

\section{Acknowledgements}

We thank Olga Ramilijaona and and Daniel Rakotondravony from the Département de Biologie Animale de l'Université d'Antananarivo, and the Comission Tripartite CAFF for their authorization and support of this study. Field work was carried out under permit $\mathrm{N}^{\circ}$ 95/MINEVEF.EF/SG/DGEF/DPB/SCBLF/RECH and $\mathrm{N}^{\circ} 21 / \mathrm{MIN}$ EVEF.EF/SG/DGEF/DPB/SCBLF/RECH granted from the Ministère de l'Environnement des Eaux et Fôrets. We thank Christiane Denys and Jacques Cuisin (MNHN, Paris), Chris Smeenk (Naturalis, Leiden), and Paula Jenkins and Richard C. Sabin (NHM, London), Robert Asher and Frieder Mayer (ZMB, Berlin) and Katrin Krohmann (Senckenberg, Frankfurt) for kindly granting us access to the collections of the respective museums and we thank Steven Goodman and José Myriel Ralison for additional tissue samples. Eildert Groeneveld is thanked for providing the computational resources needed for the MCMC-based analyses. For assistance with GIS in creating the map of the sampling sites we thank Dietmar Zinner. This study was supported by the German Science Foundation (DFG KA 1082/8-1 and -2) and the German Primate Center (DPZ). 


\section{Genetic structure, lack of sex-biased dispersal and behavioral flexibility in the pair-living fat-tailed dwarf lemur, Cheirogaleus medius}

Tina Fredsted ${ }^{1} \&$ Mikkel H. Schierup ${ }^{1} \&$ Linn F. Groeneveld ${ }^{2} \&$ Peter M. Kappeler $^{2,3}$

${ }^{1}$ Department of Ecology and Genetics, Institute of Biological Sciences, University of Aarhus, Ny Munkegade, building 540, 8000 Århus C, Denmark

${ }^{2}$ Department of Behavioral Ecology and Sociobiology, German Primate Center, Kellnerweg 4, 37077 Göttingen, Germany

${ }^{3}$ Institute of Zoology und Anthropology, University of Göttingen, 37073 Göttingen, Germany

Behavioural Ecolology Sociobiology (2007) 61:943-954 / Received: 7 July 2006 / Revised: 10 November 2006 / Accepted: 25 November 2006 / Published online: 10 January 2007 / Springer-Verlag 2007 


\begin{abstract}
Mating system and dispersal patterns influence the spatio-genetic structure within and between populations. Among mammals, monogamy is rare, and its socio-genetic consequences have not been studied in detail before. The goal of our study was to investigate population history, demographic structure, and dispersal patterns in a population of pair-living fat-tailed dwarf lemurs, Cheirogaleus medius, a small, nocturnal primate from western Madagascar, and to infer their underlying behavioral mechanisms. Tissue samples for DNA extraction were obtained from a total of 140 individuals that were captured in two subpopulations about $3 \mathrm{~km}$ apart. Analyses of mtDNA variability at the population level revealed very low levels of genetic variability combined with high haplotype diversity, which is indicative of a recent population bottleneck. We found no evidence for spatial clustering of same-sexed individuals with identical haplotypes within each of two subpopulations but significant clustering between them. Thus, a high level of local subpopulation differentiation was observed $\left(F_{\mathrm{ST}}=0.230\right)$. The sexes showed equal variances in the number of individuals representing each haplotype, as well as equal levels of aggregation of identical haplotypes. Hence, both sexes disperse from their natal area, one pattern expected in a pair-living mammal. There is a possibility of behavioral and social flexibility in this species, however, because we documented pronounced differences in density and sex ratio between the two subpopulations, suggesting that single study sites or populations may not be representative of a given local population or even species.
\end{abstract}

\title{
5.1 Introduction
}

The mating system that evolves in a given species can be regarded as the outcome of a conflict between males and females (Parker, 1979, Chapman et al., 2003). Because of the constraints imposed by internal gestation and subsequent lactation by females, male mammals have fewer opportunities to provide parental care than males in other taxa, so that social monogamy, where a male and a female bond and share parental care, has only been described in about $5 \%$ of species, including greater white-toothed shrews (Favre et al. 1997), giant jumping rats (Sommer, 2001), prairie voles (Roberts et al. 1998), Kirk's dik-dik (Brotherton and Rhodes, 1996), cotton-top tamarins (Ziegler and Snowdon, 2000), and gibbons (Reichard, 1995). However, pair-living does not necessarily equate with a monogamous mating system (Kappeler and van Schaik, 2002) because the level of extra-pair paternity (EPP) can be high (e.g., Alpine marmots, 31.4\% (Goossens et al., 1998, Cohas et al., 2006), fat-tailed dwarf lemurs, $44 \%$ (Fietz et al., 2000), and fork-marked lemurs, $75 \%$ (Schülke et al., 2004)); many pair-living species are indeed socially monogamous and genetically polygamous. Because the social and mating system can be decoupled, the evolutionary routes towards this type of social organization are still not fully understood, and the evolutionary interactions among paternal care, resource access, and sex-specific reproductive, as well as dispersal, strategies remain important topics of ongoing research (Dunbar, 1995, Brotherton and Komers, 2003, Reichard, 2003, van Schaik and Kappeler, 2003).

Whereas the evolutionary forces favoring mammalian monogamy continue to be discussed in some detail, its social and genetic consequences have remained relatively obscure. Dispersal and philopatry are of key importance in this respect (Perrin and Mazalov, 2000). Given the high prevalence of polygamy, most mammals are characterized by female philopatry and male dispersal (Greenwood, 1980). For monogamous species, the theoretical expectation is either 
of no sex bias or of female-biased dispersal. No sex bias is expected when local competition affects males and females equally (Perrin and Mazalov, 2000). On the other hand, in some monogamous species, males not only help rear the young, but they may also acquire and defend resources to attract a female (Favre et al., 1997). Males, therefore, should be more philopatric, being the sex that benefits most from familiarity with their natal area. Hence, female-biased dispersal is an equally plausible possibility in monogamous species. An example is provided by the greater white-toothed shrew (Favre et al., 1997), and Fietz et al. (2000) reported indications of female dispersal in the fat-tailed dwarf lemur.

The distribution of genotypic variation (genetic structure) in a population can be used to obtain information about a given dispersal pattern (Chesser, 1991). This approach is particularly useful in small, nocturnal, or cryptic species, where direct observations of dispersal events are extremely difficult. Dispersal ability will be inversely correlated with the degree of genetic differentiation (Cowlishaw and Dunbar, 2000). For example, if genetic structure is similar in both sexes, unbiased dispersal can be an explanation. On the other hand, if matrilines or brotherhoods of closely related members of the same sex are observed, sexbiased dispersal can be inferred, as members of the resident sex become closely related over time, especially in small social units (e.g., Altmann, 1990, Lukas et al., 2005). A tendency for male-biased dispersal and matrilines has been observed in a wide range of mammals (e.g., Lehman et al., 1992, Girman et al., 1997, Surridge et al., 1999, Fernando and Lande, 2000), including primates such as Coquerel's dwarf lemur and gray mouse lemurs (Kappeler et al., 2002, Wimmer et al., 2002, Fredsted et al., 2004). However, genetic structure has, to our knowledge, not yet been used to illuminate dispersal patterns in a pair-living mammal.

Aspects of the mating system can also be investigated using genetic information. The mating system affects the effective population size $\left(\mathrm{N}_{\mathrm{e}}\right)$, and $\mathrm{N}_{\mathrm{e}}$ can be estimated using the level of genetic variation. For example, studies in birds revealed that genetic diversity is higher in populations with a higher level of EPP (Petrie et al., 1998). In addition, $\mathrm{N}_{\mathrm{e}}$ decreases when the variance in reproductive success among individuals increases in a population Hartl and Clark, 1989). In polygynous species, where some males are much more likely to contribute genetically to the next generation than others, this variance is very large in males, and hence, this can lead to very low $\mathrm{N}_{\mathrm{e}}$ (Hedrick, 2005). In promiscuous and monogamous species, the variance in males is lower, resulting in a larger $\mathrm{N}_{\mathrm{e}}$ (Chesser, 1991). Furthermore, mating systems with polyandrous females result in an increase in effective population sizes above those expected under polygyny and monogamy (Sugg and Chesser, 1994). Hence, if extrapair copulations are shown to occur regularly within a socially monogamous species, male variance decreases (female variance is assumed to stay approximately the same), and this can increase $\mathrm{N}_{\mathrm{e}}$ (Sugg and Chesser, 1994).

The primates of Madagascar (Lemuriformes) represent a wide spectrum of social and mating systems (Kappeler, 1997). Even closely related sympatric and ecologically similar species differ in this respect. Whereas the social and genetic consequences of the social systems of nonmonogamous lemurs have been studied in some detail, information about dispersal patterns and genetic structure in pair-living species is still lacking. The nocturnal fat-tailed dwarf 
lemur, Cheirogaleus medius (130 g), from the dry deciduous forests of western Madagascar is one of the best-studied pair-living lemurs (Bourlière and Petter-Rousseaux, 1966, Petter, 1988, Dausmann et al., 2000). Cheirogaleus medius lives in small family groups, consisting of a reproducing male-female pair and their offspring from one or more previous breeding seasons (Müller, 1998, Fietz, 1999b). Pair-partners were reported to cooperate in taking care of their young (Fietz, 1999b). Males and females appear to maintain prolonged pair bonds, mate monogamously, and usually separate only when one partner dies (Fietz and Dausmann, 2003). Their social system is therefore similar to that seen in many birds, most canids, as well as some other primates, such as gibbons, tamarins, and marmosets.

Despite social monogamy, high levels of EPP (44\%; Fietz et al., 2000) have been detected in the only previous genetic study of $C$. medius, so that an increased $\mathrm{N}_{\mathrm{e}}$ is expected. A test of this prediction is hampered by a lack of conclusive information about dispersal patterns in $C$. medius, however. The floating males described by Fietz et al. (2000) suggest male dispersal. However, the genetic results of their study indicated female dispersal and male philopatry. Furthermore, at another study site, no evidence for floating males was found (Müller and Thalmann, 2002). The aims of our study, therefore, were to use genetic data to measure dispersal, to characterize historical and present demography, and to relate these variables to the social system in order to illuminate cause and effect of the breeding system and to infer the behavioral mechanisms underlying it.

\subsection{Materials and methods}

\subsubsection{Trapping and sample collection}

Sampling was conducted at the research station of the German Primate Centre in Kirindy Forest, a dry-deciduous forest located about $60 \mathrm{~km}$ northeast of Morondava in western Madagascar $\left(40^{\circ} 40^{\prime} \mathrm{E}, 20^{\circ} 4\right.$ 'S). Capturing was performed in two subpopulations, named N5 (25 ha) and CS7 (30 ha), situated approximately $3 \mathrm{~km}$ apart. Using Sherman life traps placed every $25 \mathrm{~m}$ at the intersections of the two trail grid systems in these subpopulations, a total of 140 fat-tailed dwarf lemurs were captured over the course of three seasons from October-April: 1999/2000, 16 individuals; 2000/2001, 28 individuals; and 2004/2005, 96 individuals. In total, 82 of these individuals were captured in N5 and 48 in CS7, and the remaining 10 were obtained from transects outside these study grids. The traps were placed in the vegetation at a height of 40-200 $\mathrm{cm}$. In the late afternoon, they were opened and baited with small pieces of banana and checked early in the morning. Trapping was performed in the grid systems for three consecutive nights to capture most of the individuals present. Recapture rates from earlier studies of the same and sympatric species have shown that recapture rates after the third night are very high, so that this trapping scheme should sample a very large fraction of the total population.

Captured animals were sexed and individually marked with a subdermally injected transponder. Because C. medius has extremely seasonal life histories (they hibernate between 
May and October; (Dausmann et al., 2004)), individuals can be unambiguously assigned to a particular age cohort. Because they reach sexual maturity during their first year of life (Foerg, 1982), we considered all individuals as adults, those who were captured during or after the second season after their birth, i.e., when they were at least about 20 months old. In addition, small $\left(2-3 \mathrm{~mm}^{2}\right)$ ear biopsies were taken during brief anesthesia, and the tissue samples were immediately transferred to $70 \%$ ethanol. The lemurs were released at their capture site in the subsequent late afternoon.

\subsubsection{Sequencing}

DNA was extracted using the Qiagen DNeasy tissue kit (Chatsworth, CA, cat. no. 69504). A 530-bp fragment of the mtDNA D-loop (control region) was amplified via polymerase chain reaction using the mammalian control region primers L15997 5'-CAC ATT AGC ACC CAA AGC T-3' located in the tRNA gene and H16498 5'-CCT GAA GTA GGA ACC AGA TG-3' (Gerloff et al. 1999 ). In a $10 \mu \mathrm{l}$ reaction, $1 \mu \mathrm{l}$ buffer (1.5 mM MgCl2), $1.6 \mu$ l deoxyribonucleotide triphosphate (1.25 mM of A, C, G, T, respectively), $0.5 \mu \mathrm{l}$ of each primer $(10 \mathrm{pmol} / \mu \mathrm{l})$, $1 \mu \mathrm{l}(20-60 \mathrm{ng} / \mu \mathrm{l})$ template, and $0.1 \mu \mathrm{l}$ Taq polymerase were used. Cycling conditions were as follows: an initial denaturation step of $3 \mathrm{~min}$ at $94^{\circ} \mathrm{C}$, followed by 40 cycles of $30 \mathrm{~s}$ at $94^{\circ} \mathrm{C}$, $40 \mathrm{~s}$ at $60^{\circ} \mathrm{C}, 1 \mathrm{~min}$ at $72^{\circ} \mathrm{C}$, and a final extension step of $7 \mathrm{~min}$ at $72^{\circ} \mathrm{C}$. Sequencing was conducted under BigDy ${ }^{\mathrm{TM}}$ terminator cycling conditions, the reacted products purified using ethanol precipitation and run using an Automatic Sequencer ABI 3730xl. Both strands were sequenced in all samples.

A 378-bp fragment of the mitochondrial cytochrome oxidase subunit I (COI) was also sequenced in a subset of six individuals (primers: For-5'-CCC CGA ATA AAC AAT ATA AGC TTC TG-3'; Rev-5'-AAA AAT CAG AAT AGG TGT TGG TAT AG-3').

\subsubsection{Population genetic analyses}

Sequences were analyzed using BIoEDIT version 7.0.0 (Hall, 1999). Identical haplotypes among the 140 sequences were found using the Collapse tool written by Villesen (http:// www.daimi.au.dk/ biopv/research/php/fabox.php). Diversity measures of the D-loop were estimated as both haplotype diversity $(h)$ and nucleotide diversity ( $\pi$; Nei 1978) using DNASP version 4.10.3 (Rozas et al., 2003). Haplotype diversity is measured by the total number of differences recorded in all possible pairwise comparisons of the haplotypes, and nucleotide diversity is the average proportion of nucleotide differences between all possible pairs of sequences in the sample. These measures provide information about the level of variation in the population and can thereby give indications of demographic history, population size (effective), and mutation rates.

To test whether the probability of dispersal is similar for both sexes, the mean and variance of the number of representatives of each haplotype were compared between all males and females. If both sexes disperse, no significant difference in the variance is expected. As the variances were larger than the means, the data were log-transformed before applying an $F$-test 


\section{(Lewontin, 1966).}

The relationship between haplotypes was displayed as a minimum spanning network using TCS version 1.21 (Clement et al. 2000). This program uses an absolute distance matrix for all pairwise comparisons of haplotypes, calculates the parsimony connection limit, and using these justified connections, calculates a (by default) $95 \%$ set of plausible networks (statistical parsimony network).

To verify that the $C$. medius COI sequences fall into the known main primate clades at the expected position, a phylogenetic tree of the six COI sequences and additional COI sequences from 19 primate species retrieved from GenBank was constructed with MEGA version 3.1 (Kumar et al., 2004), using the minimum evolution criterion and the HKY substitution model.

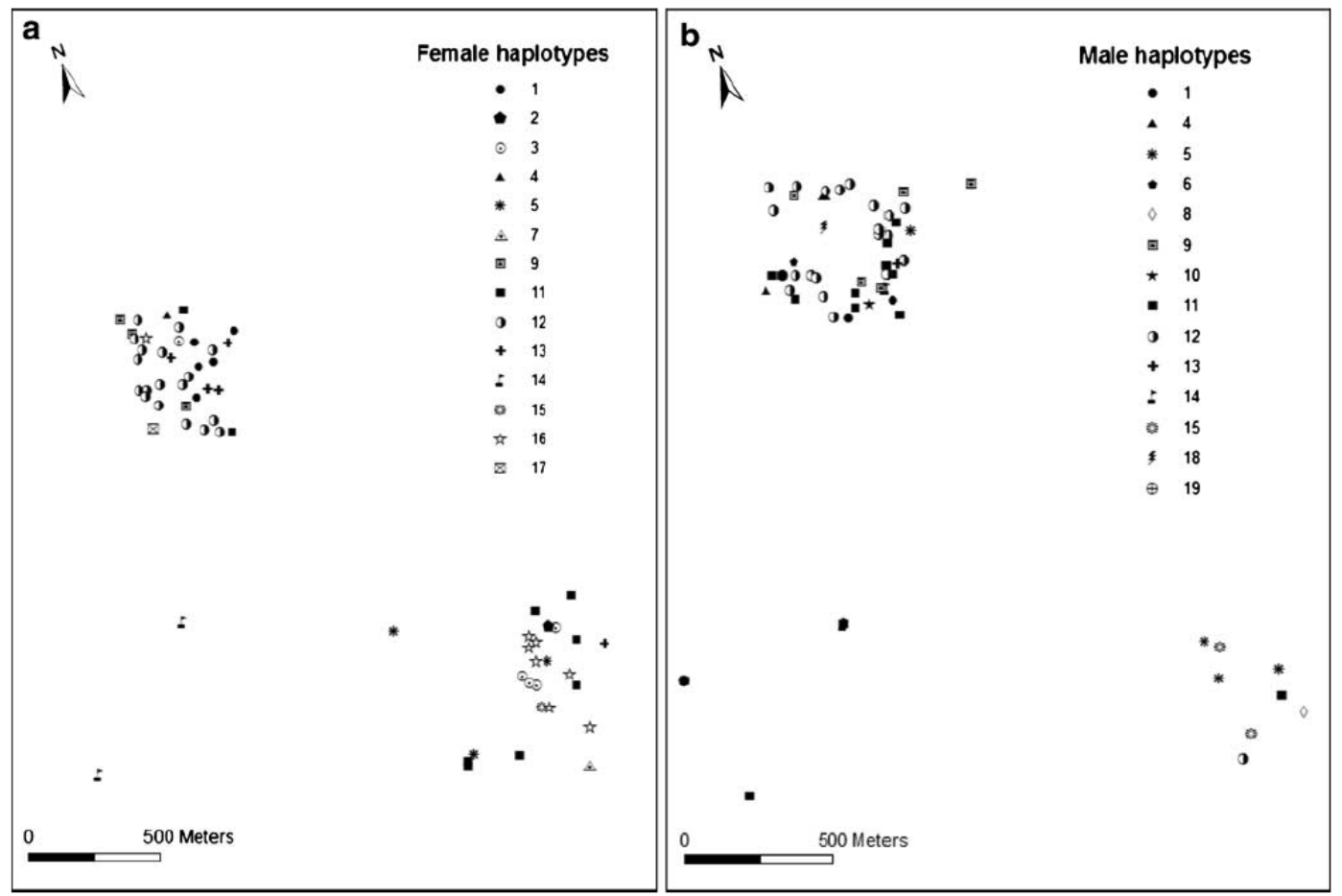

Figure 5.1: Map of the study area and geographic distribution of mtDNA haplotypes (a females and $\mathbf{b}$ males). Each symbol represents an adult individual dwarf lemur with its mtDNA haplotype at the mean capture site. The map was generated in ARCVIEw GIS. Two geographical outliers located 4 kilometers east of the other samples are not shown on the two maps, as this would remove the resolution of the remaining samples; one male (35) and one female (29). The female outlier has a unique haplotype (20) and hence there is one haplotype missing in the female map (14 in map 1a, 15 in females total). Subadults $(N=14)$ were not included in the maps.

\subsubsection{Demographic analyses}

We evaluated the influence of past demographic events or selective pressures on the frequency and spatial distribution of haplotypes with two commonly used summary statistics of the 


\section{Sex specific haplotype distribution}

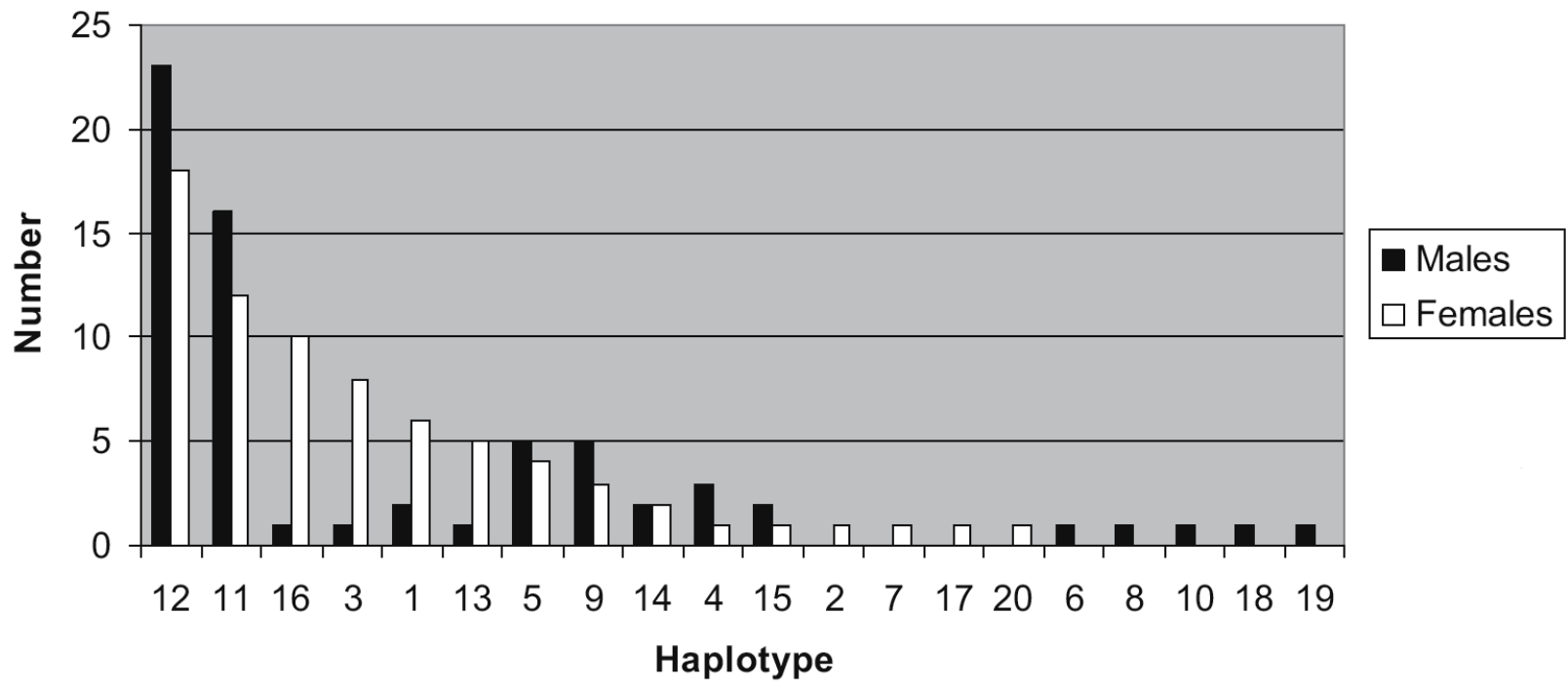

Figure 5.2: Frequency distribution of the haplotypes found in males (black) and females (white).

within-population allele frequency distribution: (1) Tajima's $D$, which compares two estimators of the population mutation rate based on the number of segregating sites $(\theta)$ and average nucleotide diversity ( $\pi$; Tajima 1989) and (2) Fu and Li's $D$, which is based on the differences between the number of singletons (mutations appearing only once among the sequences) and the total number of mutations ( $\mathrm{Fu}$ and $\mathrm{Li}, 1993)$. If a population is panmictic and stable in size, the expectation of these quantities is zero. Deviations from this expectation are therefore informative about the evolutionary and demographic forces that a population has experienced. For example, negative values reflect an excess of rare polymorphisms in a population, which is consistent with either positive selection or an increase in population size (population expansion). Positive values indicate an excess of alleles with intermediate frequency in a population and can result from either balancing selection or population fusion.

We also conducted mismatch distribution analyses, using DNASP, where the number of sequence differences between pairs of alleles (haplotypes) are plotted and such distributions have characteristic shapes for populations with different demographic histories (e.g., stable, exponential growing, bottleneck, secondary contact; Avise, 2000, Frankham et al., 2002b). For example, bottlenecks yield either a bimodal distribution or a distribution close to zero (L-shaped), depending on whether the bottleneck reduced genetic diversity, or completely removed it (so that the diversity represents mutations from that point). Humans exhibit a unimodal distribution characteristic of exponential growth.

\subsubsection{Spatial analyses}

A distribution map of the capture sites of all adult individuals, labeled with their respective haplotypes and sex, was created using ArCVIEw GIS version 9.0 (Fig. 5.1). We exam- 
ined the spatial distribution of adult individuals with three different methods. First, using Spatial Genetic Software (Degen et al., 2001), we tested for haplotype clumpiness in each sex separately by calculating an aggregation index. The value of $R$ in the aggregation index indicates whether there is a random distribution $([R]=1)$ or an aggregated, clumped distribution $([R]<1)$. The statistical significance level of all measures was determined by a Monte Carlo permutation test (Manly, 1997). Second, using $F_{\text {STAT }}$, an estimate of $F_{\text {ST }}$ (genetic variance statistics measuring the genetic divergence among populations) by Weir and Cockerham (1984) was calculated between the two subpopulations for each sex separately. Third, we examined the spatial distribution of individuals with different haplotypes using a permutation test (10,000 permutations). We compared the mean distances among same-sexed individuals with identical haplotypes with distributions of mean distances among same-sexed individuals with different haplotypes. This was done in the total population as well as in each subpopulation separately. A ratio $<1$ indicates spatial clustering of individuals with identical haplotypes (see Fredsted et al., 2005 for details).

\subsection{Results}

\subsubsection{Genetics}

A total of 30 segregating sites, defining 20 different haplotypes, were found in 140 sequenced individuals (Table 5.1. GenBank, accession numbers DQ410650-DQ410669). Sampling details and statistics are summarized in Table 5.1, which also features a comparison with closely related, sympatric Microcebus murinus, a solitary and promiscuous lemur for which the same types of data are available from an earlier study (Fredsted et al., 2004). BLASTing (BLAST, basic local alignment search tool) the sequence against GenBank revealed highest similarity to other lemurs. However, this sequence differed from other lemurs enough to support the high rate of evolution of the D-loop.

The complete frequency distribution of haplotypes is shown for both sexes separately in Fig. 5.2. Males and females did not differ in haplotype number (males/females, 16:15), and the sexes showed approximately equal numbers of unique haplotypes (males/females, 5:4; Fig. 5.2). The mean number of representatives of each haplotype did not differ for males and females, and the variances of the number of representatives were not significantly different $(t=-0.469, P$ [same mean] $=0.642, F=1.060, P$ [same variance] $=0.900 ;$ males $/$ females mean $=0.910 / 1.042$, variance $=0.816 / 0.770)$. These conclusions did not differ when we included subadults in the analyses (data not shown).

The minimum spanning network shows the frequency of each haplotype and the number of changes among haplotypes (Fig. 5.3). Two major clades are present with a deeper split between haplotypes 5-8 and the rest connected through haplotype 11. There are two very common haplotypes $(11,12)$. Furthermore, with the few mutational changes present between all haplotypes in the network, the very low level of variation found in this population is illustrated (Table 5.1). Moreover, haplotype 20, represented by only one individual, could 
Table 5.1: Comparison of the genetic polymorphism data between fat-tailed dwarf lemurs ( C. medius, this study) and the closely related sympatric gray mouse lemur ( $M$. murinus; see Fredsted et al., 2004).

\begin{tabular}{|c|c|c|}
\hline & Cheirogaleus medius & Microcebus murinus \\
\hline \# Individuals & 140 & 205 \\
\hline Sex ratio (males:females) & $66: 74$ & 102:102 (1 not sexed $)$ \\
\hline \# Haplotypes & 20 & 22 \\
\hline \# Variable sites & 30 & 69 \\
\hline \# Singletons & 13 & 3 \\
\hline \# Informative sites & 17 & 66 \\
\hline Nucleotide diversity, $\pi$ & $0.0051(=0.51 \%)$ & $0.0424(=4.24 \%)$ \\
\hline Pairwise haplotype divergence & $0.20-2.90 \%$ & $0.19-8.20 \%$ \\
\hline Haplotype diversity $(h)$ & 0.855 & 0.806 \\
\hline Tajima's $D^{a}$ & $-1.48, P>0.10$ & $0.47, P>0.10$ \\
\hline Fu and Li's $D^{a}$ & $-2.72, P<0.05$ & $0.15, P>0.10$ \\
\hline Aggregation index ${ }^{a}$ (males/females) & $0.154, P<0.001 / 0.153, P<0.001$ & $0.228, P<0.001 / 0.135, P<0.001$ \\
\hline$F_{\mathrm{ST}}($ males/females $)$ & $0.225 / 0.233$ & $\sim 0 / 0.202$ \\
\hline
\end{tabular}

Variable sites refer to sites that vary between sequences, singletons are mutations appearing only once among the sequences, and informative sites are mutations occurring in more than one sequence. Pairwise haplotype divergence refers to the percentage pairwise difference between all different haloptypes (excluding identical ones). The remaining measures are described in "Material and Methods". "Statistical significance levels were determined by permutation tests.

not be linked to the others, unless the parsimony connection limit was lowered to $93 \%$. In fact, the sequence of this individual was very different from the remaining individuals and its haplotype accounted for as many as 8 of the 13 singletons in the complete data set.

\subsubsection{Demography}

The two subpopulations differed in density and sex ratio. In N5, a total of 82 C. medius were caught, whereas 48 were caught in the similar-sized CS7 area. The sex-ratio (M/F) of sampled individuals was 0.33 in CS7 and 1.34 in N5. Hence, there was an excess of males in N5 and a large deficiency of males in CS7, suggesting that even closely located subpopulations can have very different structure.

The test statistics that are informative about the evolutionary and demographic forces a population has experienced (Tajima's $D$ and Fu and Li's $D$ ) were both negative, Fu and Li's $D$ significantly so (Table 5.1), which reflects an excess of rare polymorphisms. This is consistent with either positive selection or population expansion. The mismatch distribution analysis revealed a positively skewed distribution with a bimodal tendency and a 'wave' in the beginning, which is indicative of a recent population bottleneck (Fig. 5.4). 


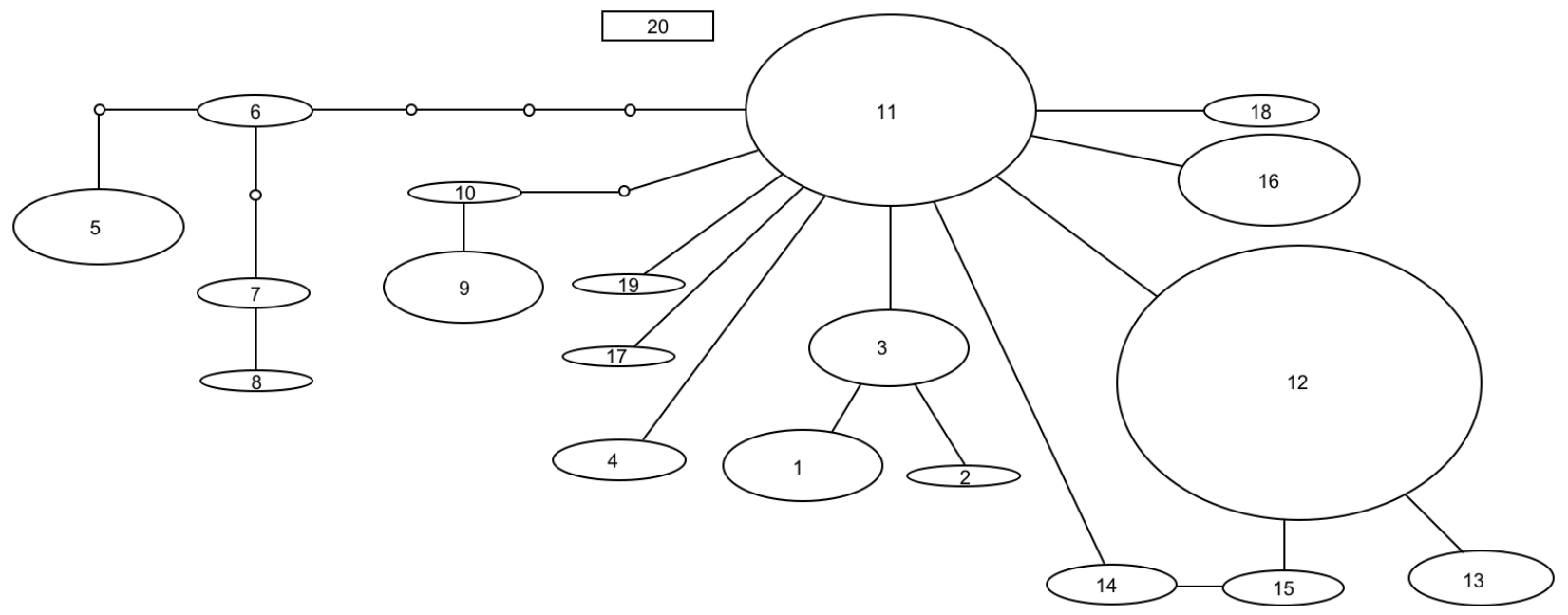

Figure 5.3: Minimum spanning network (statistical parsimony network). Haplotypes are drawn in a size proportional to their frequency (number of individuals harboring that haplotype). The small nodes represent number of mutational differences between haplotypes in excess of one. This network shows two very common haplotypes $(11,12)$. Furthermore, the few mutational changes present between all haplotypes in the network illustrate the very low level of variation found in this study.

\subsubsection{Spatial patterns}

The spatial distribution of adult males and females with different haplotypes is shown in Fig. 5.1. Visual inspection suggested spatial aggregation of identical haplotypes, which was confirmed by the significant aggregation index and was similar in both sexes (males: $R=0.153$, $P<0.001$; females: $R=0.154, P<0.001) . \quad F_{S T}$ estimates were large and almost identical in both sexes (males $=0.229$ and females $=0.230$ ), showing clear differentiation in both males and females between the two subpopulations. The permutation test gave overall (total population) ratios significantly $<1$ in both females $(0.41, P \sim 0.0037)$ and males $(0.78, P<0.0001)$. Hence, in both sexes, there was a significant clustering between subpopulations of individuals with identical haplotypes, whereas within subpopulations, there was no significant clustering (N5 males $=1.00, P=0.247$; N5 females $=0.93, P=0.087$; CS7 males $=0.85, P=0.222$; CS7 females $=1.11, P=0.689)$. Hence, there are groups of females/males with identical haplotypes that are only found in either one of the subpopulations, and the mean distance between identical haplotypes is much smaller than the mean distance to other haplotypes (both for males and females). This clustering was not created by parents-offspring associations because subadults $(\mathrm{N}=14)$ were excluded from the analyses.

\subsubsection{Cytochrome oxidase subunit I}

The combined facts that the D-loop sequence of C. medius was very different from that of other primates and that this species exhibited very low levels of polymorphism prompted us to sequence another, more conserved, region of the mitochondrion, the COI, in a small set of six 


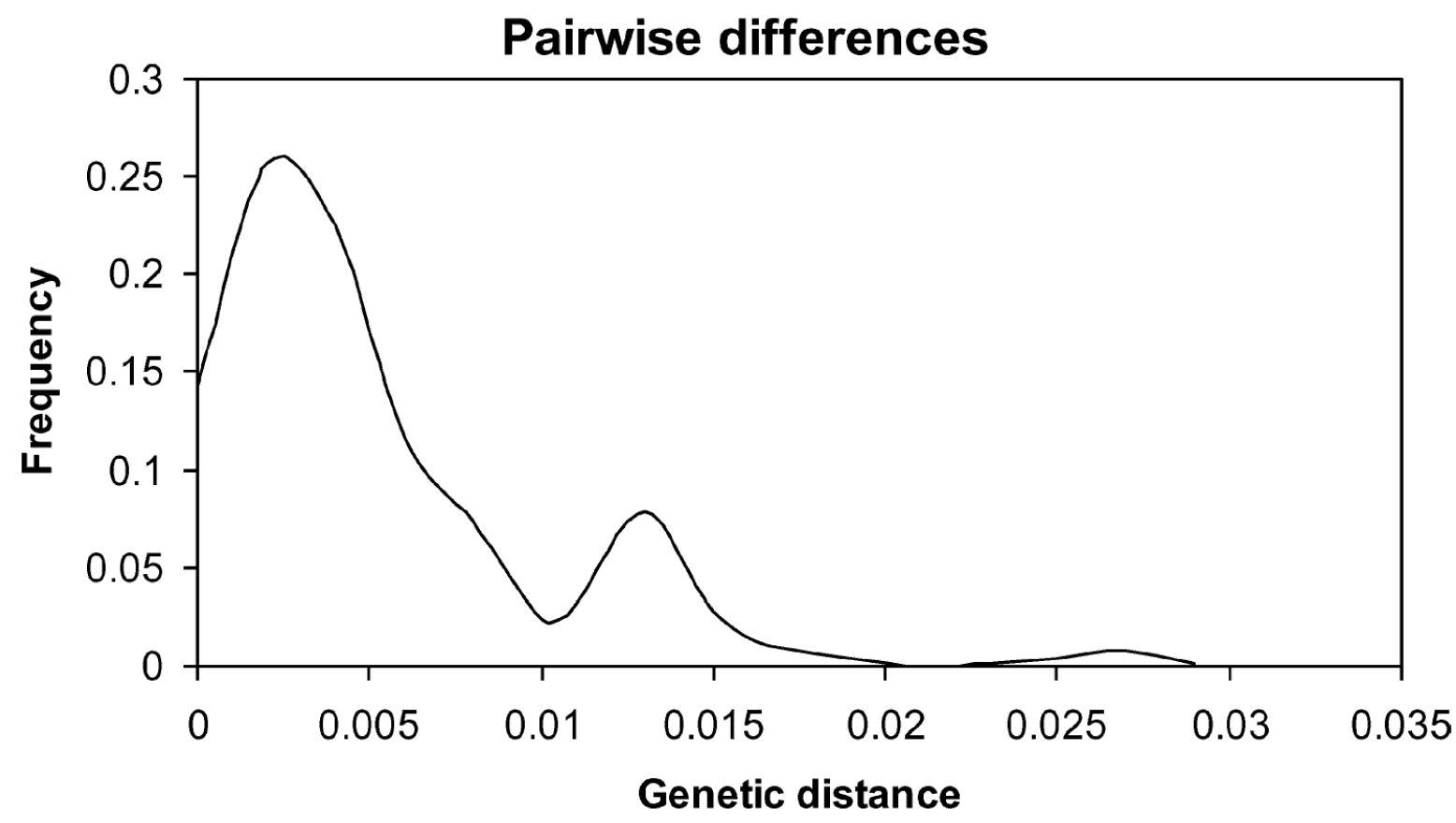

Figure 5.4: Mismatch distributions of pairwise sequence differences between haplotypes. Such distributions have characteristic shapes for populations with different demographic histories. This positively skewed distribution with a bimodal tendency and a 'wave' in the beginning indicates a recent bottleneck and subsequent expansion.

individuals representing different D-loop haplotypes. The main reason was to guard against the possibility that the D-loop sequences were part of a nuclear mitochondrial DNA (NUMT), in which case, a low level of variation would be expected. In total, we found three segregating sites defining four haplotypes, a nucleotide diversity of 0.0027 , and a Tajima's $D$ value of -1.23 , all in line with the D-loop results. Sequences from COI for 19 additional primate species were retrieved from GenBank and used to construct the phylogenetic tree shown in Fig. 5.5. The reason for creating this phylogeny was to test if the C. medius COI sequences fall into the known main primate clades at the expected position. This was confirmed, as seen in Fig. 5.5 .

\subsection{Discussion}

The most important results of this study are that (1) there is no evidence for significant sex-biased dispersal but instead dispersal by both sexes to the same degree, (2) even close subpopulations can have different dynamics, indicating behavioral and social flexibility, and (3) the population of this pair-living primate has experienced a recent population bottleneck followed by population expansion, leading to loss of genetic diversity. 


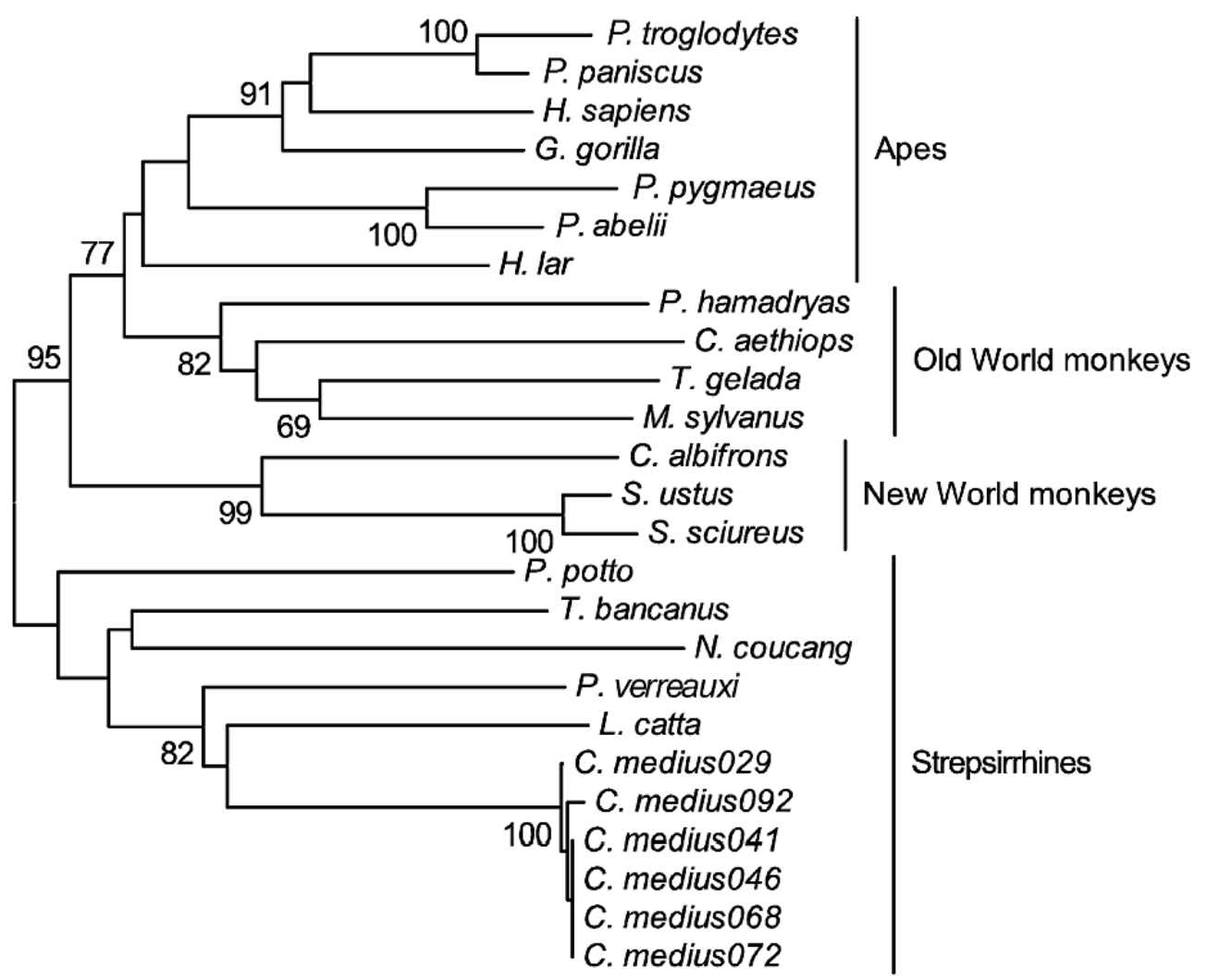

0.05

Figure 5.5: Phylogenetic tree of the COI sequences. The tree was reconstructed using the minimum evolution criterion and the HKY substitution model. Bootstrap values $>60 \%$ are shown. The six C. medius individuals were sequenced in this study and the 19 additional primate sequences were obtained from GenBank. Major primate groups are indicated on the right hand side of the tree as strepsirrhines, New World monkeys, Old World monkeys and Apes. The known main primate clades are supported and the $C$. medius COI sequences fall into the tree at the expected position.

\subsubsection{Genetic variability and logical prerequisites}

D-loop sequences of $C$. medius exhibited very low levels of variation $(\pi=0.5 \%)$. This point is illustrated by a comparison with corresponding chimpanzee, gorilla, and human Dloop nucleotide diversity, which is between 4 to almost 20 times higher than the estimate seen here (chimpanzees 7.5\%, gorilla 9.9\%, humans 2.0\%; Wise et al., 1997). Variation in chimpanzees and humans has already been considered to be very low (Yu et al. 2003). Our previous results from a similar study of sympatric $M$. murinus showed more than twice the number of polymorphic sites, and the nucleotide diversity was more than eight times higher (Table 5.1 . Fredsted et al., 2004).

This low variation, together with the lack of available sequences from closely related species, therefore prompted us to test the possibility that the D-loop sequences actually represent NUMTs by sequencing the COI region in selected individuals. The logic behind this approach 
was that it is unlikely that the same NUMT should be recognized by an independent primer set for a different gene. Because mitochondria are approximately 1,000 times more common than nuclear DNA molecules in the cell, it is unlikely that both D-loop and COI are not of mitochondrial origin. The Dloop is expected to be more variable than COI and, therefore, higher variation in COI should be a cause for concern. However, because very low levels of variation were also observed in COI, as well as a negative Tajima's $D$, the phylogeny of the COI fits with our prior knowledge of primate phylogeny, and finally, we did not obtain more than a single sequence for any individual, we conclude that the D-loop sequences are indeed of mitochondrial origin and that our results therefore do not represent an artifact.

\subsubsection{Population biology}

We suggest that the low level of variation and excess of low frequency variation is compatible with demographic changes in the form of a recent population bottleneck followed by population expansion. The combination of low nucleotide diversity and high haplotype diversity is often contributed to genetic bottleneck events with subsequent population expansion (Alves et al., 2001, Trizio et al., 2005). A population bottleneck of moderate severity is likely to cause loss of diversity, leaving only very few different lineages (in this case perhaps two), and subsequent growth establishes long terminal branches in the phylogeny, as evidenced by an excess of singletons arising from the few haplotypes left (Hein et al. 2005). Remembering that bottlenecks may yield either a bimodal distribution or a distribution close to zero (L-shaped), depending on whether the bottleneck reduced genetic diversity, or completely removed it, the positively skewed mismatch distribution with a bimodal tendency obtained in this study supports the interpretation that this $C$. medius population has undergone a bottleneck and subsequent expansion (the latter generating the 'wave' in the distribution (Rogers and Harp-

ending, 1992)). Given the number of singletons, few mutational changes between haplotypes (Fig. 5.5) and the mutation rate of the D-loop of mtDNA $\left(\sim 10^{-6}-10^{-7}\right)$, the proposed bottleneck must have been quite recent, most likely within the last 200 years. Because the study population inhabits a continuous block of forest and because C. medius is not a hunted species, the cause of a potential bottleneck remains obscure. Perhaps natural fluctuations in population density, as documented in sympatric Mirza coquereli (Kappeler et al., 2002), are sufficient to elicit such effects. Dispersal must have been a key proximate mechanism in mediating this population dynamic, i.e., recolonization of empty suitable habitat.

\subsubsection{Population dynamics of sympatric species}

Given the fairly recent population bottleneck suggested by our analyses, it is interesting to compare relevant aspects of population genetic structure with that of sympatric species, which should show similar patterns, if the cause of the bottleneck was ecological. As small populations retain less variation than larger ones, the observed very low nucleotide diversity $(\pi=0.51 \%)$ in $C$. medius indicates that this species has a very low effective population size, much smaller than closely related and sympatric $M$. murinus $(\pi=4.24 \%)$ that have already 
been studied in similar detail (Fredsted et al. 2004, Table 5.1). The observed lower nucleotide diversity in C. medius was not due to errors in sampling design because the number of haplotypes in the two studies was similar (20 vs. 22 ). The difference suggests that demographic events have had a much greater impact on $\mathrm{N}_{\mathrm{e}}$ in C. medius.

In general, species with larger body sizes usually have lower population densities due to larger home range requirements (Clutton-Brock and Harvey, 1978). Furthermore, they also usually have slower growth rates and longer generation times (Bonner, 1965), making them more vulnerable to disturbance. These factors will all often influence $\mathrm{N}_{\mathrm{e}}$ negatively, and therefore, the lower $\mathrm{N}_{\mathrm{e}}$ in the twice as large C. medius may not be surprising. The smaller proportion of singletons indicates that a recent bottleneck has not occurred in M. murinus. The diversity measures, Tajima's $D$ and Fu and Li's $D$, were both negative for C. medius; $\mathrm{Fu}$ and Li's significantly so, again in contrast to M. murinus, where these indices were not significantly different from zero. This result provides no evidence for either population fusion or any selective pressures, indicating that sequences evolved neutrally (Table 5.1, Fredsted et al., 2004). Hence, closely related sympatric species of similar size and ecology can apparently undergo bottlenecks independently of each other.

\subsubsection{Genetic structure and dispersal patterns}

Further comparison revealed a difference in the physical distribution of the genetic variation and highlighted sex differences in dispersal between the two sympatric species. In M. murinus, females had a larger aggregation index and $F_{\mathrm{ST}}$ than males, and the permutation test of clumping of individuals with identical haplotype was only significant in females, all indicating higher male mobility (Fredsted et al., 2004). In C. medius, the equal level of aggregation, the almost equal $F_{\mathrm{ST}}$ estimates, and the significant clumping of individuals with identical haplotypes in both sexes indicate that dispersal is in fact not significantly female-biased, as suggested by Fietz et al. (2000), as the maternally inherited mtDNA should result in less aggregation of related females and a smaller $F_{\mathrm{ST}}$ in that case. Furthermore, variance in the number of representatives of each haplotype was similar in males and females, which is expected if both sexes disperse. Given the sampling method, which was assumed to sample a very large fraction of the total $C$. medius population, these results cannot be explained by any other behavioral mechanism than dispersal of both sexes to the same degree.

These genetic differences between solitary and promiscuous M. murinus and pair-living and monogamous C. medius make sense in the light of their contrasting social system. No sex bias in dispersal is expected when local competition equally affects males and females, as can happen in monogamous systems (Perrin and Mazalov, 2000). Hence, given the basically monogamous mating system in C. medius, no sex difference in dispersal rates can be expected, and the analyses performed here support this prediction. Hence, we conclude that both sexes are likely to disperse in pair-living C. medius. Genetic or behavioral evidence for dispersal by both sexes in other pair-living primates is rare (Moore, 1984, Pusey, 1992, Smale et al., 1997). Bisexual dispersal occurs regularly in a number of non-female bonded species, such as 
gorillas, some colobines and New World primates (e.g., Robbins, 1995, Pope, 2000, Steenbeek et al. 2000), but its genetic consequences have only been studied in a few cases (Faulkes et al. 2003 Huck et al., unpublished data).

Dispersal in C. medius seems to occur to a rather limited degree, creating local populations that are potentially differentiated behaviorally from nearby populations. The two subpopulations, N5 and CS7, differed in density and sex ratio and seem to have different dynamics, suggesting the existence of fine-scaled processes and local adaptations that remain to be examined in detail. However, this observation echoes our earlier conclusion based on a study of M. murinus over similar spatial scales (Fredsted et al., 2004) that any randomly chosen single study site may not be representative for the social system of a local population, or even species, as it does not provide adequate information to describe species-level patterns and dynamics.

Such intraspecific behavioral variation is not uncommon in mammals (Lott, 1991) but is has rarely been documented at such a small spatial scale (cf., Travis et al., 1995, Yamagiwa and Hill, 1998, Schulte-Hostedde and Millar, 2004, Schradin and Pillay, 2005). The observed large differences in density and sex ratio in the two C. medius subpopulations are surprising given the fact that they are situated only about $3 \mathrm{~km}$ apart. The excess of males observed in N5 is concordant with the pattern observed during an earlier study there by Fietz et al. (2000), suggesting a temporal stability to the pattern. The observed spatial heterogeneity in genetic and population structure is also surprising because our study also suggested some cases of long distance migration. In both populations, we found several single haplotypes in both sexes as well as one unusual haplotype obtained about $5 \mathrm{~km}$ away from one of the study subpopulations. Because another individual captured at the same site had the second most common haplotype, this pattern is difficult to interpret without additional behavioral data on actual dispersal events. For the time being, we can only speculate that the limited time available for preparation for hibernation may limit options for long-distance dispersal in this species. The causes for pronounced and stable sex ratio imbalances remain completely obscure, however, especially given that the closely related M. murinus dispose of mechanisms for adaptive sex ratio manipulation (Perret, 1996).

\subsection{Conclusions}

In conclusion, our study provided evidence for equal dispersal by both sexes in a pair-living primate species. This dispersal seems to occur to a limited degree, creating local populations that are potentially quite differentiated behaviorally from nearby populations. We discovered population differences in density and sex ratio at the smallest spatial scale, indicating behavioral and social flexibility in this species, which suggests that any randomly chosen study site may not be representative for the social system of a local population, or even species. Comparison with a closely related sympatric species demonstrated that differences in social organization and dispersal patterns result in different genetic structures. 


\section{Acknowledgments}

We thank Joanna Fietz and Kathrin Daussmann for providing additional genetic samples. We also thank the late Madame Berthe Rakotosamimanana at the Département de Paléontologie et d'Anthropologie Biologique de l'Université d'Antananarivo, the late Albert Randrianjafy and Gilbert Rakotoarisoa of Parc Botanique et Zoologique de Tsimbazaza, Madame Olga Ramilijaona and Daniel Rakotondravony of the Département Biologie Animale de l'Université d'Antananarivo, the members of the Commission Tripartite and the C.A.F.F. of the Direction des Eaux et Forêts, and the CFPF Morondava for their authorization and support of this study. We thank Palle Villesen for programming the permutation test of haplotypes in R. Also, thanks to Stine W. Bjorholm for creating the GIS map and Benoit Goossens for very helpful comments on this manuscript. The German Primate Centre and grants from the WWF Biodiversity Fund and Augustinus Fond, Denmark, financed this work. T. Fredsted was supported by a Ph.D. grant from the Faculty of Science of the University of Aarhus, Denmark. 


\section{General Conclusion}

\subsection{Summary of results}

This dissertation addressed aspects of species delimitation, phylogeography and population genetics of the endemic Malagasy genus Cheirogaleus. Morphometric, molecular and distributional data at multiple scales from all available data sources, including museum collections, sequence data from GenBank and newly collected specimens from the field, were used to answer the following questions:

1. Is the current taxonomy, which recognizes seven species, supported by new data from the field?

Morphological and genetic data are concordant in the support of the existence of three Cheirogaleus species and thus the current taxonomy is not fully supported by our analyses.

a) To what conclusion regarding the number of Cheirogaleus species does the analysis of morphological data from field and museum specimens lead?

Analyses of morphological data, from six external and 32 cranio-dental characters of 120 museum specimens and 36 individuals from the field, revealed three distinct morphs, which correspond to C. medius, C. major and C. crossleyi. Cheirogaleus adipicaudatus and C. ravus are proposed to be synonymous with $C$. medius and C. major, respectively. The sample size for C. minusculus is too small for decisive inferences and results for the status of $C$. sibreei are inconclusive.

b) What can be concluded from the analyses of mitochondrial DNA and nuclear DNA markers in terms of species delimitation?

Analyses based on mtDNA $(c y t b+c o x 2)$ and three independent nuclear markers (adora3, fiba and $v W F$ ) from 48 individuals caught in the field, 17 museum specimens and 24 haplotypes from GenBank, support three evolutionary lineages, which correspond to C. medius, C. major and C. crossleyi. Population genetic cluster analyses revealed a further layer of resolution within the three lineages and identified two distinct sets of populations/individuals per lineage.

2. How are the extant Cheirogaleus species distributed geographically and how did those patterns arise? 
The three recognized species, as assessed by the morphological and genetic analyses, are not local endemics, but have distributions spanning the island. Cheirogaleus medius is found along the west coast from the southeastern to the northeastern tip of the island. Cheirogaleus major is present along the east coast from the southeastern tip up to the Masoala peninsula and C. crossleyi is found in the eastern rainforests from the southeastern to the northernmost tip and also on the northwestern coast as far south as Ampijoroa.

a) Can existing biogeographic hypotheses for Madagascar be supported by new distributional data and a reassessed Cheirogaleus taxonomy?

Neither the model of biogeographic zones based on phytogeography, nor the 'centers-of-endemism' hypothesis is concordant with, or explains the contemporary distribution of the currently recognized species of dwarf lemurs.

b) When did the different Cheirogaleus species begin to differentiate and how do their time divergence estimates compare to closely related taxa?

The age estimates for dwarf lemur species range from 1.5 to 8.1 mya (95\% credibility intervals) and are comparable to estimates for mouse lemur species at 1.5 to 12.8 mya ( $95 \%$ credibility intervals) and thus species level divergences in both genera largely predate the Quaternary. This indicates that models, such as the 'centersof-endemism' hypothesis, which assume that climatic shifts in the Quaternary were the driving force of speciation, do not apply.

3. What can be deduced from the population genetic structure of a $C$. medius population from western Madagascar?

Evidence for equal dispersal by both sexes in a pair-living primate species, C. medius, was found. This dispersal seems to occur to a limited degree, creating local populations that are potentially quite differentiated behaviorally from nearby populations. On the one hand the confounding factor of genetic signatures due to potentially asymmetrical dispersal patterns of the sexes can thus be disregarded in future mtDNA based studies. On the other hand population differences were found on a small spatial scale and it is therefore questionable how representative the conclusions of this study are.

a) What does the present population genetic structure of a $C$. medius population in western Madagascar reveal about the demographic history of that population?

Analyses of mtDNA variability at the population level, i.e. of 140 individuals from two subpopulations, revealed very low levels of genetic variability combined with high haplotype diversity, which is indicative of a recent population bottleneck.

b) Which sex disperses in C. medius and what is the consequence in terms of population genetic structure? 
No evidence for spatial clustering of same-sexed individuals with identical haplotypes within each of two C. medius subpopulations was found, indicating equal dispersal by both sexes. Comparison with a closely related sympatric species demonstrated that differences in social organization and dispersal patterns result in different genetic structures: In Microcebus murinus females had a larger aggregation index and $\mathrm{F}_{\mathrm{ST}}$ than males, whereas in C. medius the sexes showed equal variances in the number of individuals representing each haplotype, as well as equal levels of aggregation of identical haplotypes and almost equal $\mathrm{F}_{\mathrm{ST}}$ estimates.

c) Are these data representative for the whole species?

We discovered population differences in density and sex ratio at the smallest spatial scale, indicating behavioral and social flexibility in this species, which suggests that any randomly chosen study site may not be representative for the social system of a local population, or even species.

\subsection{Main research questions regarding Madagascar's natural heritage}

Madagascar, with its unparalleled wealth of endemic flora and fauna, has been characterized as every naturalist's paradise. Even though this island has been the focus of naturalists for centuries, very basic questions, such as 'What forces led to the species-rich endemic radiations?' and 'How can this remarkable biodiversity best be saved from extinction?' remain unanswered. In the following I will examine how the results generated by this dissertation can contribute towards answering these questions.

\section{Uncovering Madagascar's evolutionary history}

Current research on the initial origins of the endemic Malagasy vertebrate radiations concludes that Madagascar was colonized by a few dispersal events in the Cenozoic (e.g. Vences, 2004, Poux et al., 2005, Yoder and Nowak, 2006). This first part of the evolutionary history of Madagascar is supported by a number of independent lines of concordant data: Within the mammals there are four major endemic terrestrial lineages, all of which are monophyletic with the respective sister group found in Africa, according to phylogenetic analyses (e.g Yoder et al., 1996, Yoder, 1997, Olson and Goodman, 2003, Yang and Yoder, 2003, Yoder et al., 2003, Jansa and Weksler, 2004, Yoder and Yang, 2004, Poux et al., 2005). Studies on the Malagasy herpetofauna, including tortoises, scincid lizards, chameleons, geckos, colubrid snakes and frogs, for the most part come to the same conclusions as for mammals (e.g. Caccone et al., 1999, Mausfeld et al., 2000, Raxworthy et al., 2002, Nagy et al., 2003, Austin et al., 2004, Vences et al., 2004). Fewer conclusive studies are available for fishes. There are persuasive indications of an ancient vicariant origin of Malagasy fresh-water fish (e.g. Sparks and Smith, 2004), but other studies have advocated a Cenozoic dispersal origin, for example for cichlids 
(Vences et al., 2001). Madagascar's extant birds, due to their ability to fly, are not surprisingly found to have arrived on Madagascar via dispersal (e.g. Cibois et al., 1999, Groombridge et al., 2002, Warren et al., 2003). Thus, there seems to be some consensus that Madagascar's extant fauna is the progeny of a few Cenozoic dispersers, but little is known what shaped the subsequent radiations.

Molecular methods are increasingly being used to elucidate historical events and analytical methods are becoming more sophisticated in estimating divergence dates, the demographic history of populations, the relationships among different lineages, the size and distribution of ancestral populations, and the extent of past migration and gene flow between populations (reviewed in e.g. Hewitt, 2004, Yoder and Nowak, 2006). If one has access to a large pool of sequence data from a range of different taxa, then one can hope to uncover common patterns that may lead to a new testable hypothesis. This approach led to the currently favored hypothesis of how Madagascar's fauna arrived on the island, as outlined above. For examining endemic radiations after colonization, this type of data is not yet sufficiently available. We are missing such basic data as clear taxonomies, distributional data of extant taxa, resolved phylogenies, and sufficient molecular data from a tight spatial sampling scheme for phylogeographic analyses, which operate at the interface of population genetics.

Further complications are introduced by the lack of Malagasy terrestrial and freshwater vertebrate fossils from the Tertiary (e.g. Simons et al., 1995, Krause et al., 1997, Krause, 2003 , de Wit, 2003). Not only would a fossil record in itself give valuable insight into the evolutionary history of the island, it would also yield data for calibrating time divergence estimates. Flynn and Wyss (2003) give us reason for hope in their account of the history of Malagasy paleontology; at lot has been learned in the past decade and there surely is more to uncover.

The type of data needed for answering how the speciose endemic radiations in Madagascar came about was completely lacking for the genus Cheirogaleus. The last taxonomic revision was based entirely on museum specimens, little distributional data was available at the specific level, age estimates of the origin of the genus, as well as the species were not available. In the framework of this dissertation, I established a robust taxonomy, added distributional data for bio- and phylogeographic analyses and estimated divergence dates. These data should be viewed as a building block for future models of Madagascar's evolutionary history.

\section{Conserving Madagascar's natural heritage}

Madagascar's flora and fauna is in dire need of protection, if it is to be saved from its plight of extinction. The loss of forest habitat has been estimated to have accelerated dramatically: in 1950,1985 and 2000 only about $65 \%, 34 \%$, and 10\%, respectively, of Madagascar's primary tropical rain forests remained (Green and Sussman, 1990, Myers et al., 2000). The firm political will to increase Madagascar's protected areas and thus to save its biodiversity is given. Madagascar's president Marc Ravalomanana declared in 2003 at the 5th World Parks Congress in Durban, South Africa his intention to triple the country's protected area coverage 
from 1.7 million hectares to 6 million hectares within the next five years. First steps in reaching this goal have been taken. By the end of 2005 and 2007 more than an additional 1 million hectares, respectively, had been officially declared protected areas (CEPF, 2006, 2007).

In order to implement effective conservation management plans, is it not only necessary to protect areas, but to have precise information on the taxa to be conserved. The field of genetics can provide a wide array of valuable information. Frankham et al. (2002a) list four groups of questions that can be answered by genetic methods, which can help to devise management plans. The first complex deals with the detail of knowledge of the taxonomy, the second with questions pertaining to the size of populations, the third with aspects of the species' biology and the fourth with illegal hunting or trade.

Within the first complex of questions the crucial task is to determine the taxonomic status of a species or population. It would have far-reaching implications for conservation, if an apparently widespread, low-risk species turned out to actually consist of a number of closely related distinct taxa. Such a situation has been described in a number of nocturnal lemurs, such as the sportive lemurs (genus Lepilemur) and mouse lemurs (genus Microcebus). This dissertation has provided the best estimate of dwarf lemur taxonomy to date. Most importantly, this study has combined museum and field data, so that species names could be designated to populations in the field. There is little merit for conservationists in knowing that a certain species exists, if it is not known where, apart from the museum drawers. Furthermore, it is of interest whether any populations within a species are sufficiently differentiated genetically to warrant separate management. The Bayesian population assignment test, used to infer population structure, revealed two distinct sets of populations/individuals, within each species. Both for C. medius and C. crossleyi the individuals from Sambava in northwestern Madagascar formed a distinct cluster. The populations at this site should thus be scrutinized carefully in future research.

The second complex of questions pertains to the size of populations, that are under consideration for conservation action. This dissertation has only touched upon this subject in the genetic study of a C. medius population in central-western Madagascar. For this particular population a very small effective population size was detected. Since small populations are more prone to a loss of genetic diversity and thus extinction, this study can serve as a first indication that the dwarf lemurs of the central-western Menabe region may require monitoring and genetic management.

The third complex deals with questions relating to the species' biology. Knowledge about migration and dispersal patterns is crucial for assessing the species' survival prospects. The population genetic study provided data, which indicated that dispersal in C. medius occurs to a rather limited degree, creating local populations that are quite differentiated from nearby populations. Moreover, the study yielded information on sex-specific dispersal, indicating that both sexes disperse equally.

The fourth question complex deals with the application of genetic methods to detect illegal hunting and trade. Since illegal hunting in Madagascar does not rely on commercial distribution of the products there is probably fairly little use for our data in this regard (García and 
Goodman, 2003). But certainly, should this become of interest, mtDNA profiles can be used to clearly determine the species identity of any dwarf lemur products.

In conclusion, this dissertation has provided some important baseline data that can be incorporated into future conservation action plans.

\subsection{Methodology}

A major part of this dissertation relied on the capture of dwarf lemurs in the field to obtain tissue samples and morphometric data. The characteristics of the biology and natural history of the members of this genus evince the difficulty of obtaining large sample sizes. Field work is only possible during the active phase of the animals, which coincides with the rainy season, thus restricting field work to about six months per year. Even though C. medius individuals at Kirindy, central-western Madagascar, are known to enter traps frequently and in large numbers, at other sites, members of this genus do not behave comparably. After not having caught a single Cheirogaleus individual during a total of 8800 trap nights at a total of eight sites, more emphasis was placed on darting the animals individually, rather than relying solely on the traps. Similar trapping problems were reported by Lahann (2007a), who noted that the trapping rates of $C$. major in comparison to the sympatrically occurring $C$. medius and Microcebus murinus were extremely low in Mandena. Unfortunately, the darting method is extremely time-consuming and cumbersome. The animals are only detectable via their eye shine and they are often up high in the canopy (Lahann, 2007b), where they move quickly and agilely. Furthermore, this method restricted us to three individuals per site, which we were permitted to sacrifice as morphotypes. The danger of severe injury due to the immobilisation darts, is too high, in order to use this method to capture and subsequently release the animals.

Due to the low sample number, numerous analyses, especially those operating at the interface of population genetics and phylogeography, were not feasible. As a result, the available data did not contain sufficient signal, or did not fulfill the assumptions of the respective models.

\subsection{Outlook}

As a result of this dissertation, the following areas for future research can be outlined.

1. Assessment of species identity of to date unclassified populations, using both morphometric and genetic data. This includes the populations identified in the Makira region by Rasolofoson et al. (2007), the C. major like morphs in the Bongolava region as described by Thalmann and Rakotoarison (1994), Ausilio and Raveloanrinoro (1998) and Thalmann (2000), and further populations that remained unsampled by this dissertation, such as the C. medius labeled population at Daraina (Mittermeier et al., 1994). These data could be incorporated into future phylogeographic analyses and would serve as baseline data for conservation management planning. 
2. Assessment of species identity of C. minusculus type specimens using genetic data. This is currently not possible because NHM regulations do not permit any sampling of holotype material for molecular analyses. This would be the best option for deciding whether C. minusculus should retain specific status, should the NHM change its policy.

3. Closer examination of the sympatric C. medius and C. crossleyi population at Sambava. These populations were identified as clearly differentiated from other populations of the same species. They could, therefore represent populations that deserve to be managed as separate units in the conservation context.

4. Generation of additional nuclear sequence data for Cheirogaleus, Microcebus, Allocebus, Phaner and Mirza. In order to gain a more robust estimate of species level divergences within the Cheirogaleidae, additional sequence data are required. Should the estimates become congruent with those estimated by Horvath et al. (2008), the conclusions regarding the applicability of the 'centers-of-endemism' hypothesis drawn in this dissertation, would have to be revised.

5. Collection of data on ecological, behavioral, physiological and general life history traits for different populations of the three species. Little is known about dwarf lemur biology, especially of C. major and C. crossleyi. Moreover, it should be assessed whether some older studies are referring to the correct species, in terms of the reassessed taxonomy. For example, two studies that were carried out in an area where according to this dissertation $C$. crossleyi is present (although in one case not exclusively), refer to $C$. major as their taxon of concern (Ganzhorn, 1988, 1989, Wright and Martin, 1995). Data on species' biology are important for conservation as outlined above.

6. Collection of additional dwarf lemur samples in the framework of a dense sampling scheme, for future molecular analyses. Phylogeographic analyses, which need a higher number of samples per population and a denser net of sampling sites, may help to uncover the evolutionary history of the genus. Potentially these data could help to determine the processes that led to the species-rich radiations on the island.

7. Establishment of Y-chromosomal markers. A full suite of markers would thus be available for the assessment of potential hybridization or introgression events. 


\section{Bibliography}

Adkins, R. M. and Honeycutt, R. L. (1994). Evolution of the primate cytochrome $c$ oxidase subunit II gene. Journal of Molecular Evolution, 38(3):215-231.

Agapow, P.-M., Bininda-Emonds, O. R. P., Crandall, K. A., Gittleman, J. L., Mace, G. M., Marshall, J. C., and Purvis, A. (2004). The impact of species concept on biodiversity studies. The Quarterly Review of Biology, 79(2):161-179.

Agassiz, L. (1845). Nomenclator Zoologicus. Fasciculus VII et VIII, Mammalia: Addenda et corrigenda. Jent et Gassmann.

Agassiz, L. (1846). Nomenclatoris zoologici: Index universalis. Jent et Gassmann.

Altmann, J. (1990). Primate males go where the females are. Anim Behav, 39:193-195.

Alves, M., Coelho, H., Collares-Pereira, M., and Coelho, M. (2001). Mitochondrial DNA variation in the highly endangered cyprinid fish Anaecypris hispanica: Importance for conservation. Heredity, 87(4):463-473.

Andriaholinirina, N., Fausser, J.-L., Roos, C., Zinner, D., Thalmann, U., Rabarivola, C., Ravoarimanana, I., Ganzhorn, J., Meier, B., Hilgartner, R., Walter, L., Zaramody, A., Langer, C., Hahn, T., Zimmermann, E., Radespiel, U., Craul, M., Tomiuk, J., Tattersall, I., and Rumpler, Y. (2006). Molecular phylogeny and taxonomic revision of the sportive lemurs (Lepilemur, Primates). BMC Evolutionary Biology, 6(1).

Andriantompohavana, R., Zaonarivelo, J., Engberg, S., Randriamampionona, R., McGuire, S., Shore, G., Rakotonomenjanahary, R., Brenneman, R., and Louis, E. (2006). Mouse lemurs of northwestern Madagascar with a description of a new species at Lokobe Special Reserve. Occasional Papers, Museum of Texas Tech University, 259:1-24.

Ausilio, E. and Raveloanrinoro, G. (1998). Les Lémuriens de la région de Bemaraha: Forêts de Tsimembo, de l'Antsingy et de la région de Tsiandro. Lemur News, 3:4-7.

Austin, J. J., Arnold, E. N., and Jones, C. G. (2004). Reconstructing an island radiation using ancient and recent DNA: The extinct and living day geckos (Phelsuma) of the Mascarene islands. Molecular Phylogenetics and Evolution, 31(1):109-122.

Avise, J. (1987). Intraspecific phylogeography: The mitochondrial DNA bridge between population genetics and systematics. Annual Review of Ecology and Systematics, 18:489-522. 
Avise, J. (1996). Space and time as axes in intraspecific phylogeograhpy. In Huntley, B., Cramer, W., Morgan, A., Prentice, H., and Allen, J., editors, Past and Future Rapid Environmental Changes: The Spatial and Evolutionary Responses of Terrestrial Biota, pages 381-388, Berlin. Springer-Verlag.

Avise, J. (1998). The history and purview of phylogeography: A personal reflection. Molecular Ecology, 7:371-379.

Avise, J. (2000). Phylogeography: The history and formation of species. Harvard University Press, Cambridge.

Blackith, R. and Reyment, R. (1971). Multivariate Morphometrics. Academic Press Inc. (London) Ltd.

Bonner, J. (1965). Size and Cycle. Princeton University Press, USA.

Bourlière, F. and Petter-Rousseaux, A. (1966). Existence probable d'un rythme metabolique saisonnier chez les Cheirogaleinae (Lemuroidea). Folia Primatologica, 4:249-256.

Briggs, J. (2003). The biogeographic and tectonic history of India. Journal of Biogeography, $30(3): 381-388$.

Brotherton, P. and Komers, P. (2003). Mate guarding and the evolution of social monogamy in mammals. In Reichard, U. and Boesch, C., editors, Monogamy: Mating Strategies and Partnerships in Birds, Humans and other Mammals, pages 42-58. Cambridge University Press, Cambridge.

Brotherton, P. and Rhodes, A. (1996). Monogamy without biparental care in a dwarf antelope. Proceedings of the Royal Society B: Biological Sciences, 263(1366):23-29.

Burney, D. (1997). Theories and facts regarding Holocene environmental change before and after human colonization. In Goodman, S. and Patterson, B., editors, Natural Change and Human Impact in Madagascar, pages 75-89. Smithsonian Institution Press.

Burney, D., Burney, L., Godfrey, L., Jungers, W., Goodman, S., Wright, H., and Jull, A. J. (2004). A chronology for late prehistoric Madagascar. Journal of Human Evolution, 47(1$2): 25-63$.

Burney, D., Robinson, G., and Burney, L. (2003). Sporormiella and the late Holocene extinctions in Madagascar. Proceedings of the National Academy of Sciences, 100(19):1080010805 .

Burney, D. A. (1987). Late Holocene vegetational change in central Madagascar. Quaternary Research, 28(1):130-143.

Caccone, A., Amato, G., Gratry, O. C., Behler, J., and Powell, J. R. (1999). A molecular phylogeny of four endangered Madagascar tortoises based on mtDNA sequences. Molecular Phylogenetics and Evolution, 12(1):1-9. 
CEPF (2006). Assessing five years of CEPF investment. Critical Ecosystem Partnership Fund, a Special Report.

CEPF (2007). Protecting nature's hotspots for people and prosperity. Critical Ecosystem Partnership Fund, Annual Report.

Chapman, T., Arnqvist, G., Bangham, J., and Rowe, L. (2003). Sexual conflict. Trends in Ecology and Evolution, 18(1):41-47.

Chesser, R. (1991). Influence of gene flow and breeding tactics on gene diversity within populations. Genetics, 129:573-583.

Cibois, A., Pasquet, E., and Schulenberg, T. S. (1999). Molecular systematics of the Malagasy babblers (Passeriformes: Timaliidae) and warblers (Passeriformes: Sylviidae), based on cytochrome $b$ and 16S rRNA sequences. Molecular Phylogenetics and Evolution, 13(3):581595.

Clement, M., Posada, D., and Crandall, K. (2000). TCS: A computer program to estimate gene genealogies. Molecular Ecology, 9:1657-1659.

Clutton-Brock, T. and Harvey, P. (1978). Mammals, resources and reproductive strategies. Nature, 273(5659):191-195.

Cohas, A., Yoccoz, N., Da Silva, A., Goossens, B., and Allainé, D. (2006). Extra-pair paternity in the monogamous alpine marmot (Marmota marmota): The roles of social setting and female mate choice. Behavioral Ecology and Sociobiology, 59(5):597-605.

Cowlishaw, G. and Dunbar, R. (2000). Primate Conservation Biology. The University of Chicago Press, Chicago.

Cracraft, J. (1983). Species concepts and speciation analysis. In Johnston, R., editor, Current Ornithology, volume 1, chapter 6, pages 159-187. Plenum Press.

Craul, M., Zimmermann, E., Rasoloharijaona, S., Randrianambinina, B., and Radespiel, U. (2007). Unexpected species diversity of Malagasy primates (Lepilemur spp.) in the same biogeographical zone: a morphological and molecular approach with the description of two new species. BMC Evolutionary Biology, 7(1):83.

Cuvier, F. (1842). Table générale et méthodique de toutes les espèces figurées et décrite dans l'histoire naturelle des mammifères. In Geoffroy Saint-Hilaire, E. et Cuvier, F., editor, Histoire Naturelle des Mammifères, avec Figures Originales, Coloriées, Dessinées d'aprés des Animaux Vivants, volume IV et le Tome VII de l'Histoire Naturelle des Mammifères, page 2. Blaise.

Darroch, J. and Mosimann, J. (1985). Canonical and principal components of shape. Biometrika, 72:241-252. 
Darwin, C. (1859). On the Origin of Species by Means of Natural Selection: Or the Preservation of Favored Races in the Struggle for Life. John Murray, London.

Dausmann, K., Ganzhorn, J., and Heldmaier, G. (2000). Body temperature and metabolic rate of a hibernating primate in Madagascar: Preliminary results from a field study. In Heldmaier, G. and Klingenspor, M., editors, Life in the Cold, pages 41-48. Springer, Berlin.

Dausmann, K., Glos, J., Ganzhorn, J., and Heldmaier, G. (2004). Physiology: Hibernation in a tropical primate. Nature, 429(6994):825-826.

de Queiroz, K. (1998). The general lineage concept of species, species criteria and the process of speciation. In Howard, D. and Berlocher, S., editors, Endless Forms: Species and Speciation, chapter 5. Oxford University Press.

de Queiroz, K. (2005). Ernst Mayr and the modern concept of species. Proceedings of the National Academy of Sciences, USA, 102(suppl. 1):6600-6607.

de Wit, M. J. (2003). Madagascar: Heads it's a continent, tails it's an island. Annual Review of Earth and Planetary Sciences, 31(1):213-248.

Degen, B., Petit, R., and Kremer, A. (2001). SGS-Spatial genetic software: A computer program for analysis of spatial genetic and phenotypic structures of individuals and populations. Journal of Heredity, 92(5):447-448.

Degnan, J. H. and Rosenberg, N. A. (2006). Discordance of species trees with their most likely gene trees. PLoS Genetics, 2(5).

Donoghue, M. J. and Moore, B. R. (2003). Toward an integrative historical biogeography. Integrative and Comparative Biology, 43(2):261-270.

Drummond, A. J. (2007). BEAST: Bayesian evolutionary analysis by sampling trees. BMC Evolutionary Biology, 7:214.

Drummond, A. J., Ho, S. Y. W., Phillips, M. J., and Rambaut, A. (2006). Relaxed phylogenetics and dating with confidence. PLoS Biology, 4(5):e88.

Du Puy, D. and Moat, J. (1995). A refined classification of the primary vegetation of Madagascar based on the underlying geology: Using GIS to map its distribution and to assess its conservation status. In Lourenço, W., editor, Proceedings of the International Symposium on the 'Biogéographie de Madagascar', pages 205-218.

Dunbar, R. (1995). The mating system of callitrichid primates: I. Conditions for the coevolution of pair bonding and twinning. Animal Behaviour, 50(4):1057-1070.

Dunn, C. (2003). Keeping taxonomy based in morphology. Trends in Ecology and Evolution, $18(6): 270-271$. 
Durbin, J. (1999). Lemurs as flagships for conservation in Madagascar. In Rakotosamimanana, B., Rasamimanana, H., Ganzhorn, J., and Goodman, S., editors, New Directions in Lemur Studies. Kluwer Academic/Plenum Publishers.

Elliot, D. (1913). A Review of the Primates, volume 1. American Museum of Natural History, New York. Monograph No. 1.

Evanno, G., Regnaut, S., and Goudet, J. (2005). Detecting the number of clusters of individuals using the software STRUCTURE: A simulation study. Molecular Ecology, 14:2611-2621.

Faulkes, C., Arruda, M., and Monteiro Da Cruz, M. (2003). Matrilineal genetic structure within and among populations of the cooperatively breeding common marmoset, Callithrix jacchus. Molecular Ecology, 12(4):1101-1108.

Favre, L., Balloux, F., Goudet, J., and Perrin, N. (1997). Female-biased dispersal in the monogamous mammal Crocidura russula: Evidence from field data and microsatellite patterns. Proceedings of the Royal Society B: Biological Sciences, 264(1378):127-132.

Fernando, P. and Lande, R. (2000). Molecular genetic and behavioral analysis of social organization in the Asian elephant (Elephas maximus). Behavioral Ecology and Sociobiology, 48(1):84-91.

Fietz, J. (1999a). Demography and floating males in a population of Cheirogaleus medius. In Rakotosamimanana, B., Rasamimanana, H., Ganzhorn, J., and Goodman, S., editors, New Directions in Lemur Studies, chapter 9. Kluwer Academic/Plenum Publishers.

Fietz, J. (1999b). Monogamy as a rule rather than exception in nocturnal lemurs: The case of the fat-tailed dwarf lemur, Cheirogaleus medius. Ethology, 105:259-272.

Fietz, J. and Dausmann, K. (2003). Costs and potential benefits of parental care in the nocturnal fat-tailed dwarf lemur (Cheirogaleus medius). Folia Primatologica, 74(5-6):246258.

Fietz, J. and Ganzhorn, J. (1999). Feeding ecology of the hibernating primate Cheirogaleus medius: How does it get so fat? Oecologia, 121(2):157-164.

Fietz, J., Zischler, H., Schwiegk, C., Tomiuk, J., Dausmann, K., and Ganzhorn, J. (2000). High rates of extra-pair young in the pair-living fat-tailed dwarf lemur, Cheirogaleus medius. Behavioral Ecology and Sociobiology, 49:8-17.

Flynn, J. and Wyss, A. (2003). Mesozoic terrestrial vertebrate faunas: The early history of Madagascar's vertebrate diversity. In Goodman, S. and Benstead, J., editors, The Natural History of Madagascar, pages 34-40. The University of Chicago Press.

Foerg, R. (1982). Reproduction in Cheirogaleus medius. Folia Primatologica, 39(1-2):49-62.

Forsyth Major, C. (1896). Diagnosis of new mammals from Madagascar. The Annals and Magazine of Natural History, 6(18):318-325. 
Frankham, R., Ballou, J., and Briscoe, D. (2002a). Introduction to Conservation Genetics. Cambridge University Press, Cambridge.

Frankham, R., Ballou, J., and Briscoe, D. (2002b). Use of molecular genetics in forensics and to understand species biology. In Introduction to Conservation Genetics, chapter 19, pages 471-501. Cambridge University Press, Cambridge.

Fredsted, T., Pertoldi, C., Olesen, J., Eberle, M., and Kappeler, P. (2004). Microgeographic heterogeneity in spatial distribution and mtDNA variability of gray mouse lemurs (Microcebus murinus, Primates: Cheirogaleidae). Behavioral Ecology and Sociobiology, 56(4):393403.

Fredsted, T., Pertoldi, C., Schierup, M., and Kappeler, P. (2005). Microsatellite analyses reveal fine-scale genetic structure in grey mouse lemurs (Microcebus murinus). Molecular Ecology, 14(8):2363-2372.

Fu, Y. and Li, W. (1993). Statistical tests of neutrality of mutations. Genetics, 133(3):693709.

Ganzhorn, J. (1988). Food partitioning among Malagasy primates. Oecologia, 75(3):436-450.

Ganzhorn, J. (1989). Niche separation of seven lemur species in the eastern rainforest of Madagascar. Oecologia, 79(2):279-286.

Ganzhorn, J. (1998). Nested patterns of species composition and their implications for lemur biogeography in Madagascar. Folia Primatologica, 69(suppl. 1):332-341.

Ganzhorn, J., Goodman, S., Nash, S., and Thalmann, U. (2006). Lemur biogeography. In Lehman, S. and Fleagle, J., editors, Primate Biogeography, chapter 8, pages 229-254. Springer, New York.

García, G. and Goodman, S. M. (2003). Hunting of protected animals in the Parc National d'Ankarafantsika, north-western Madagascar. Oryx, 37(1):115-118.

Gelman, A. and Rubin, D. (1992). Inference from iterative simulation using multiple sequences. Statistical Science, 7:457-511.

Geoffroy Saint-Hilaire, E. (1812). Note sur trois dessins de Commerçon, représentant des quadrumanes d'un genre inconnu. Annales du Muséum d'Histoire Naturelle, 19:171-175.

Gerloff, U., Hartung, B., Fruth, B., Hohmann, G., and Tautz, D. (1999). Intracommunity relationships, dispersal pattern and paternity success in a wild living community of bonobos (Pan paniscus) determined from dna analysis of faecal samples. Proceedings of the Royal Society B: Biological Sciences, 266(1424):1189-1189.

Girman, D., Mills, M., Geffen, E., and Wayne, R. (1997). A molecular genetic analysis of social structure, dispersal, and interpack relationships of the African wild dog (Lycaon pictus). Behavioral Ecology and Sociobiology, 40(3):187-198. 
Glaw, F. and Vences, M. (1994). Locality gazetteer and locator map. In A Fieldguide to the Amphibians and Reptiles of Madagascar, pages 468-475. M. Vences \& F. Glaw Verlags GbR, second edition.

Gloger, C. (1842). Gemeinnütziges Hand- und Hilfsbuch der Naturgeschichte. Für gebildete Leser aller Stände, besonders für die reifere Jugend und ihre Lehrer, volume 1, enthaltend die erste Hälfte der Naturgeschichte der Thiere. Verlag von Aug. Schulz und Comp., Breslau.

Godfrey, L., Jungers, W., Simons, E., Chatrath, P., and Rakotosamimanana, B. (1999). Past and present distributions of lemurs in Madagascar. In Rakotosamimanana, B., Rasamimanana, H., Ganzhorn, J., and Goodman, S., editors, New Directions in Lemur Studies. Kluwer Academic/Plenum Publishers.

Goodman, S. and Ganzhorn, J. (2004a). Biogeography of lemurs in the humid forests of Madagascar: The role of elevational distribution and rivers. Journal of Biogeography, 31:4755.

Goodman, S. and Ganzhorn, J. (2004b). Elevational ranges of lemurs in the humid forests of Madagascar. International Journal of Primatology, 25(2):331-350.

Goodman, S. M. and Benstead, J. P. (2005). Updated estimates of biotic diversity and endemism for Madagascar. Oryx, 39(01):73-77.

Goossens, B., Graziani, L., Waits, L., Farand, E., Magnolon, S., Coulon, J., Bel, M.-C., Taberlet, P., and Allainé, D. (1998). Extra-pair paternity in the monogamous alpine marmot revealed by nuclear DNA microsatellite analysis. Behavioral Ecology and Sociobiology, 43(4):281-288.

Graur, D. and Li, W. (2000). Dynamics of genes in populations. In Graur, D. and Li, W., editors, Fundamentals of Molecular Evolution, chapter 2, pages 39-65. Sinauer Associates, Inc., second edition.

Gray, J. (1872). Notes on Propithecus, Indris, and other lemurs (Lemurina) in the British Museum. Proceedings of the Scientific Meetings of the Zoological Society of London, pages 846-860. Part III.

Green, G. and Sussman, R. (1990). Deforestation history of the eastern rain forests of Madagascar from satellite images. Science, 248(4952):212 - 215.

Greenwood, P. (1980). Mating systems, philopatry and dispersal in birds and mammals. Animal Behaviour, 28(4):1140-1162.

Groombridge, J. J., Jones, C. G., Bayes, M. K., van Zyl, A. J., Carrillo, J., Nichols, R. A., and Bruford, M. W. (2002). A molecular phylogeny of African kestrels with reference to divergence across the Indian Ocean. Molecular Phylogenetics and Evolution, 25(2):267-277. 
Groves, C. (2000a). The genus Cheirogaleus: Unrecognized biodiversity in dwarf lemurs. International Journal of Primatology, 21(6):943-962.

Groves, C. (2000b). What, if anything, is taxonomy? Gorilla Journal, 21:12-15.

Groves, C. (2001). Why taxonomic stability is a bad idea, or why are there so few species of primates (or are there?). Evolutionary Anthropology, 10:192-198.

Hall, T. (1999). BioEdit: A user-friendly biological sequence alignment editor and analysis program for Windows 95/98/NT. Nucleic Acids Symposium Series, 41:95-98.

Hapke, A., Fietz, J., Nash, S., Rakotondravony, D., Rakotosamimanana, B., Ramanamanjato, J.-B., Randria, G., and Zischler, H. (2005). Biogeography of dwarf lemurs: Genetic evidence for unexpected patterns in southeastern Madagascar. International Journal of Primatology, 26(4):873-901.

Hartl, D. and Clark, A. (1989). Random genetic drift: Effective population size. In Principles of Population Genetics, pages 61-95. Sinauer Associates, Sunderland, MA.

Hay, W., DeConto, R., Wold, C., Wilson, K., Voigt, S., Schulz, M., Wold-Rossby, A., Dullo, W.-C., Ronov, A., Balukhovsky, A., and Söding, E. (1999). Alternative global Cretaceous paleogeography. In Barrera, E. and Johnson, C., editors, Evolution of the Cretaceous OceanClimate System, Special Paper 332, chapter 1, pages 1-47. Geological Society of America.

Heckman, K., Mariani, C., Rasoloarison, R., and Yoder, A. (2007). Multiple nuclear loci reveal patterns of incomplete lineage sorting and complex species history within western mouse lemurs (Microcebus). Molecular Phylogenetics and Evolution.

Heckman, K., Rasoazanabary, E., Machlin, E., Godfrey, L., and Yoder, A. (2006). Incongruence between genetic and morphological diversity in Microcebus griseorufus of Beza Mahafaly. BMC Evolutionary Biology, 6(98).

Hedrick, P. (2005). Large variance in reproductive success and the $\mathrm{Ne} / \mathrm{N}$ ratio. Evolution, 59(7):1596-1599.

Hein, J., Schierup, M., and Wiuf, C. (2005). Extensions to the basic coalescent. In Gene genealogies, Variation and Evolution: a Primer in Coalescent Theory, pages 104-106. Oxford University Press, Oxford.

Hewitt, G. (2004). The structure of biodiversity - insights from molecular phylogeography. Frontiers in Zoology, 1(4).

Hey, J. (2001). The mind of the species problem. Trends in Ecology and Evolution, 16(7):326329.

Hey, J., Waples, R. S., Arnold, M. L., Butlin, R. K., and Harrison, R. G. (2003). Understanding and confronting species uncertainty in biology and conservation. Trends in Ecology and Evolution, 18(11):597-603. 
Hladik, C., Charles-Dominique, P., and Petter, J. (1980). Feeding strategies of five nocturnal prosimians in the dry forest of the west coast of Madagascar. In Charles-Dominique, P., Cooper, H., Hladik, A., Hladik, C., Pages, E., Pariente, G., Petter-Rousseaux, A., Schilling, A., and Petter, J., editors, Nocturnal Malagasy Primates, chapter 2, pages 41-73. Academic Press Inc. (London) Ltd.

Horvath, J. E., Weisrock, D. W., Embry, S. L., Fiorentino, I., Balhoff, J. P., Kappeler, P., Wray, G. A., Willard, H. F., and Yoder, A. D. (2008). Development and application of a phylogenomic toolkit: Resolving the evolutionary history of Madagascar's lemurs. Genome Research, 0:gr.7265208-.

Huelsenbeck, J. P., Ronquist, F., Nielsen, R., and Bollback, J. P. (2001). Bayesian inference of phylogeny and its impact on evolutionary biology. Science, 294:2310-2314.

Humbert, H. (1955). Les territoires phytogéographiques de Madagascar. Leur cartographie. Année Biologique, 31:195-204.

Isaac, N., Mallet, J., and Mace, G. (2004). Taxonomic inflation: Its influence on macroecology and conservation. Trends in Ecology and Evolution, 19(9):464-469.

Jansa, S. A. and Weksler, M. (2004). Phylogeny of muroid rodents: Relationships within and among major lineages as determined by IRBP gene sequences. Molecular Phylogenetics and Evolution, 31(1):256-276.

Jenkins, P. (1987). Catalogue of Primates in the British Museum (Natural History) and elsewhere in the British Isles, Part IV: Suborder Strepsirrhini, including the Subfossil Madagascan Lemurs and Family Tarsiidae. Trustees of the British Museum (Natural History).

Jolicoeur, P. and Mosimann, J. (1960). Size and shape variation in the painted turtle. A principal component analysis. Growth, 24:339-354.

Jungers, W., Falsetti, A., and Wall, C. (1995). Shape, relative size, and size-adjustments in morphometrics. American Journal of Physical Anthropology, 38(S21):137-161.

Kappeler, P. (1990). The evolution of sexual size dimorphism in prosimian primates. American Journal of Primatology, 21(3):201-214.

Kappeler, P. (1996). Intrasexual selection and phylogenetic constraints in the evolution of sexual canine dimorphism in strepsirhine primates. Journal of Evolutionary Biology, 9(1):4365 .

Kappeler, P. (1997). Determinants of primate social organization: Comparative evidence and new insights from Malagasy lemurs. Biol Rev Camb Philos Soc, 72(1):111-151.

Kappeler, P., Rasoloarison, R., Razafimanantsoa, L., Walter, L., and Roos, C. (2005). Morphology, behaviour and molecular evolution of giant mouse lemurs (Mirza spp.) Gray, 1870, with description of a new species. Primate Report, 71:3-26. 
Kappeler, P., Wimmer, B., Zinner, D., and Tautz, D. (2002). The hidden matrilineal structure of a solitary lemur: Implications for primate social evolution. Proceedings of the Royal Society B: Biological Sciences, 269(1502):1755-1763.

Kappeler, P. M. and van Schaik, C. P. (2002). Evolution of primate social systems. International Journal of Primatology, 23(4):707-740.

Kingman, J. (1982). The coalescent. Stochastic Processes and their Applications, 13(3):235248.

Koechlin, J., Guillaumet, J.-L., and Morat, P. (1974). Flore et Végétation de Madagascar, volume 5 of Flora et Vegetatio Mundi. J. Cramer.

Krause, D. (2003). Late cretaceous vertebrates of Madagascar: A window into Gondwanan biogeography at the end of the age of dinosaurs. In Goodman, S. and Benstead, J., editors, The Natural History of Madagascar, chapter 2.3. The University of Chicago Press.

Krause, D., Hartman, J., and Wells, N. (1997). Late cretaceous vertebrates from Madagascar: Implications for biotic change in deep time. In Goodman, S. and Patterson, B., editors, Natural Change and Human Impact in Madagascar. Smithsonian Institution Press.

Kumar, S., Filipski, A., Swarna, V., Walker, A., and Hedges, S. B. (2005). Placing confidence limits on the molecular age of the human-chimpanzee divergence. Proceedings of the National Academy of Sciences, 102(52):18842-18847.

Kumar, S., Tamura, K., and Nei, M. (2004). Mega3: Integrated software for molecular evolutionary genetics analysis and sequence alignment. Briefings in Bioinformatics, 5(2):150-163.

Lahann, P. (2007a). Biology of Cheirogaleus major in a littoral rain forest in southeast Madagascar. International Journal of Primatology, 28(4):895-905.

Lahann, P. (2007b). Feeding ecology and seed dispersal of sympatric cheirogaleid lemurs (Microcebus murinus, Cheirogaleus medius, Cheirogaleus major) in the littoral rainforest of south-east Madagascar. Journal of Zoology, 271(1):88-98.

Lehman, N., Clarkson, P., Mech, L., Meier, T., and Wayne, R. (1992). A study of the genetic relationships within and among wolf packs using DNA fingerprinting and mitochondrial DNA. Behavioral Ecology and Sociobiology, 30(2):83-94.

Lesson, M.-P. (1840). Species des Mammiféres Bimanes et Quadrumanes; Suivi d'un Mémoire sur les Oryctéropes. Bailliére, J-B.

Lewontin, R. (1966). On the measurement of relative variability. Systematic Zoology, 15:141142.

Lipscomb, D., Platnick, N., and Wheeler, Q. (2003). The intellectual content of taxonomy: A comment on DNA taxonomy. Trends in Ecology and Evolution, 18(2):65-66. 
Lott, D. (1991). Intraspecific Variation in the Social System of Wild Vertebrates. Cambridge University Press, Cambridge.

Louis, E., Coles, M., Andriantompohavana, R., Sommer, J., Engberg, S., Zaonarivelo, J., Mayor, M., and Brenneman, R. (2006). Revision of the mouse lemurs (Microcebus) of eastern Madagascar. International Journal of Primatology, 27:347-389.

Lukas, D., Reynolds, V., Boesch, C., and Vigilant, L. (2005). To what extent does living in a group mean living with kin? Molecular Ecology, 14(7):2181-2196.

Maddison, D. R. and Maddison, W. P. (2002). MacClade 4.05 OS X. Analysis of phylogeny and character evolution. Technical report, Sinauer, Sunderland, Massachusetts, USA.

Maddison, W. P. (1997). Gene trees in species trees. Systematic Biology, 46(3):523-536.

Mallet, J. and Willmott, K. (2003). Taxonomy: Renaissance or tower of babel. Trends in Ecology and Evolution, 18(2):57-59.

Mancuso, D., Tuley, E., Westfield, L., Worrall, N., Shelton-Inloes, B.B. Sorace, J., Alevy, Y., and Sadler, J. (1989). Structure of the gene for human von Willebrand factor*. The Journal of Biological Chemistry, 264:19514-19527.

Manly, B. (1997). Randomisation, Bootstrap and Monte Carlo Methods in Biology. Chapman and Hall, London, 2nd edition.

Martin, R. (1972). Adaptive radiation and behavior of the Malagasy lemurs. Philosophical Transactions of the Royal Society of London B, 264:295-352.

Martin, R. (1995). Prosimians: from obscurity to extinction? In Alterman, L., Doyle, G., and Izard, M., editors, Creatures of the Dark: The Nocturnal Prosimians. Plenum Press.

Mausfeld, P., Vences, M., Schmitz, A., and Veith, M. (2000). First data on the molecular phylogeography of scincid lizards of the genus Mabuya. Molecular Phylogenetics and Evolution, 17(1):11-14.

Mayden, M. (1997). A hierarchy of species concepts: The denouement in the saga of the species problem. In Claridge, M., Dawah, H., and Wilson, M., editors, Species: the Units of Biodiversity, chapter 19, pages 381-424. Chapmann and Hall.

Mayr, E. (1947). Systematics and the Origin of Species. 3rd print. Columbia University Press, New York.

Melnick, D. J. and Hoelzer, G. A. (1993). What is mtDNA good for in the study of primate evolution? Evolutionary Anthropology: Issues, News, and Reviews, 2(1):2-10.

Mittermeier, R., Konstant, W., Hawkins, F., Louis, E., Langrand, O., Ratsimbazafy, J., Rasoloarison, R., Ganzhorn, J., Rajaobelina, S., Tattersall, I., and Meyers, D. (2006). Lemurs of Madagascar. Conservation International, second edition. 
Mittermeier, R., Konstant, W., Nicoll, M., and Langrand, O. (1992). Lemurs of Madagascar, an Action Plan for their Conservation 1993-1999. IUCN, Gland, Switzerland.

Mittermeier, R., Tattersall, I., Konstant, W., Meyers, D., and Mast, R. (1994). Lemurs of Madagascar. Conservation International, Washington, D.C.

Molnar, P., England, P., and Martinod, J. (1993). Mantle dynamics, uplift of the Tibetian plateau and the Indian monsoon. Reviews of Geophysics, 31(4):357-396.

Moore, J. (1984). Female transfer in primates. International Journal of Primatology, 5:537589.

Moritz, C. (1994). Defining 'evolutionarily significant units' for conservation. Trends in Ecology and Evolution, 9:373-375.

Müller, A. (1998). A preliminary report on the social organisation of Cheirogaleus medius (Cheirogaleidae; Primates) in north-west Madagascar. Folia Primatologica, 69:160-166.

Müller, A. (1999a). Aspects of social life in the fat-tailed dwarf lemur (Cheirogaleus medius): Inferences from body weights and trapping data. American Journal of Primatology, 49:265280 .

Müller, A. (1999b). Social organization of the fat-tailed dwarf lemur (Cheirogaleus medius) in northwestern Madagascar. In Rakotosamimanana, B., Rasamimanana, H., Ganzhorn, J., and Goodman, S., editors, New Directions in Lemur Studies, chapter 8, pages 139-158. Kluwer Academic/Plenum Publishers.

Müller, A. (1999c). The social Organisation of the Fat-tailed Dwarf Lemur, Cheirogaleus medius (Lemuriformes; Primates). PhD thesis, Universität Zürich.

Müller, A. and Thalmann, U. (2002). Biology of the fat-tailed dwarf lemur (Cheirogaleus medius E. Geoffroy 1812): New results from the field. Evolutionary Anthropology: Issues, News, and Reviews, 11(S1):79-82.

Murphy, W. J., Eizirik, E., Johnson, W. E., Zhang, Y. P., Ryder, O. A., and O'Brien, S. J. (2001). Molecular phylogenetics and the origins of placental mammals. Nature, 409(6820):614-618.

Myers, N., Mittermeier, R., Mittermeier, C., da Fonseca, G., and Kent, J. (2000). Biodiversity hotspots for conservation priorities. Nature, 403:853-858.

Nagy, Z., Joger, U., Wink, M., Glaw, F., and Vences, M. (2003). Multiple colonization of Madagascar and Socotra by colubrid snakes: Evidence from nuclear and mitochondrial gene phylogenies. Proceedings of the Royal Society of London B, 270:2613-2621.

Nichols, R. (2001). Gene trees and species trees are not the same. Trends in Ecology and Evolution, 16(7):358-364. 
Nixon, K. and Wheeler, Q. (1990). An amplification of the phylogenetic species concept. Cladistics, 6:211-223.

Oken, L. (1816). Okens Lehrbuch der Naturgeschichte. Dritter Theil, Zweite Abtheilung: Fleischthiere. Reclam, Leipzig.

Olivieri, G., Zimmermann, E., Randrianambinina, B., Rasoloharijaona, S., Rakotondravony, D., Guschanski, K., and Radespiel, U. (2007). The ever-increasing diversity in mouse lemurs: Three new species in north and northwestern Madagascar. Molecular Phylogenetics and Evolution, 43:309-327.

Olson, L. and Goodman, S. (2003). Phylogeny and biogeography of tenrecs. In Goodman, S. and Benstead, J., editors, The Natural History of Madagascar, pages 1235-1242. The University of Chicago Press.

Parker, G. (1979). Sexual selection and sexual conflict. In Blum, S. and Blum, N., editors, Sexual Selection and Reproductive Competition in Insects, pages 123-166. Academic, New York.

Pastorini, J., Thalmann, U., and Martin, R. D. (2003). A molecular approach to comparative phylogeography of extant Malagasy lemurs. Proceedings of the National Academy of Sciences, 100(10):5879-5884.

Perret, M. (1996). Manipulation of sex ratio at birth by urinary cues in a prosimian primate. Behavioral Ecology and Sociobiology, 38(4):259-266.

Perrin, N. and Mazalov, V. (2000). Local competition, inbreeding, and the evolution of sexbiased dispersal. American Naturalist, 155(1):116-127.

Petrie, M., Doums, C., and Møller, A. (1998). The degree of extra-pair paternity increases with genetic variability. Proceedings of the National Academy of Sciences, 95(16):9390-9395.

Petter, J. (1988). Contribution a l'étude du Cheirogaleus medius dans la forêt de Morondava. In Rakotovao, L., Barre, V., and Sayer, J., editors, L'Equilibre des Ecosystemes Forestièrs a Madagascar: Actes d'un Séminaire International, pages 57-60. Page Bros, Cambridge.

Petter, J.-J., Albignac, R., and Rumpler, Y. (1977). Faune de Madagascar, volume 44: Mammifères, Lémuriens (Primates, Prosimiens). ORSTOM, CNRS, Paris.

Petter-Rousseaux, A. (1980). Seasonal activity rhythms, reproduction, and body weight variations in five sympatric nocturnal prosimians, in simulated light and climatic conditions. In Charles-Dominique, P., Cooper, H., Hladik, A., Hladik, C., Pages, E., Pariente, G., Petter-Rousseaux, A., Schilling, A., and Petter, J., editors, Nocturnal Malagasy Primates, pages 137-152. Academic Press Inc. (London) Ltd. 
Pope, T. (1996). Socioecology, population fragmentation, and patterns of genetic loss in endangered primates. In Avise, J. and Hamrick, J., editors, Conservation Genetics: Case Histories from Nature, chapter 5, pages 119-159. Chapman and Hall.

Pope, T. (2000). The evolution of male philopatry in neotropical monkeys. In Kappeler, P., editor, Primate Males, pages 219-235. Cambridge University Press, Cambridge.

Posada, D. and Crandall, K. A. (1998). Modeltest: testing the model of DNA substitution. Bioinformatics, 14(9):817-818.

Poux, C., Madsen, O., Marquard, E., Vieites, D. R., de Jong, W. W., and Vences, M. (2005). Asynchronous colonization of Madagascar by the four endemic clades of primates, tenrecs, carnivores, and rodents as inferred from nuclear genes. Systematic Biology, 54(5):719-730.

Pritchard, J., Stephens, M., and Donnelly, P. (2000). Inference of population structure using multilocus genotype data. Genetics, 155:945-959.

Pusey, A. (1992). The primate perspective on dispersal. In Stenseth, N. and Lidicker, W., editors, Dispersal: Small Mammals as a Model, pages 243-259. Chapman and Hall, London.

Rabinowitz, P., Coffin, M., and Falvey, D. (1983). The separation of Madagascar and Africa. Science, 220(4592):67-69.

Rambaut, A. (2006). FigTree: Tree figure drawing tool, version 1.0. Available from $<$ http://tree.bio.ed.ac.uk/software/figtree/>, Institute of Evolutionary Biology, University of Edinburgh.

Rambaut, A. and Charleston, M. (2002). TreeEdit: Phylogenetic tree editor v1.0 alpha 10. Available from <http://evolve.zoo.ox.ac.uk/software/treeedit/main.html $>$, Department of Zoology, University of Oxford.

Rambaut, A. and Drummond, A. (2005). Tracer v1.3: MCMC trace ananlysis tool. Available from: $<$ http://tree.bio.ed.ac.uk/software/tracer/>, Institute of Evolutionary Biology, University of Edinburgh.

Rasoloarison, R., Goodman, S., and Ganzhorn, J. (2000). Taxonomic revision of mouse lemurs (Microcebus) in the western portions of Madagascar. International Journal of Primatology, 21(6):963-1019.

Rasolofoson, D., Rakotondratsimba, G., Rakotonirainy, O., Rakotozafy, L., Ratsimbazafy, J., Rabetafika, L., and Randrianarison, R. (2007). Influences des pressions anthropiques sur les lémuriens d'Anantaka, dans la partie est du plateau de Makira, Maroantsetra, Madagascar. Madagascar Conservation \& Development, 2(1):21-27.

Raxworthy, C. J., Forstner, M. R. J., and Nussbaum, R. A. (2002). Chameleon radiation by oceanic dispersal. Nature, 415(6873):784-787. 
Razafiarison, S. (2003). La construction sociale à l'épreuve du temps. In Aubert, S., Razafiarison, S., and Bertrand, A., editors, Déforestation et Systèmes Agraires à Madagascar: les Dynamiques des Tavy sur la Côte Orientale. Cirad, Cite et Fofifa, Montpellier.

Reichard, U. (1995). Extra-pair copulations in a monogamous gibbon. Ethology, 100(99-112).

Reichard, U. (2003). Monogamy: Past and present. In Reichard, U. and Boesch, C., editors, Monogamy: Mating Strategies and Partnerships in Birds, Humans and other Mammals, pages 3-25. Cambridge University Press, Cambridge.

Rensing, S. (1999). Immobilization and anesthesia of nonhuman primates. Primate Report, 55:33-38.

Richard, A. and Dewar, R. (1991). Lemur ecology. Annual Review of Ecology and Systematics, $22: 145-175$.

Robbins, M. (1995). A demographic analysis of male life history and social structure of mountain gorilla. Behaviour, 132:21-47.

Roberts, R., Williams, J., Wang, A., and Carter, C. (1998). Cooperative breeding and monogamy in prairie voles: Influence of the sire and geographical variation. Animal Behaviour, 55(5):1131-1140.

Roeser, H., Fritsch, J., and Hinz, K. (1996). The development of the crust off Dronning Maud Land, East Antarctica. In Storey, B., EC, K., and RA, L., editors, Weddell Sea Tectonics and Gondwana Break-up, Geological Society Special Publication 108, pages 243-264. The Geological Society Publishing House.

Rogers, A. and Harpending, H. (1992). Population growth makes waves in the distribution of pairwise genetic differences. Molecular Ecology and Evolution, 9(3):552-569.

Ronquist, F. (1996). Diva version 1.1. Computer program and manual available by anonymous FTP from Uppsala University. ftp.uu.se or ftp.systbot.uu.se.

Ronquist, F. and Huelsenbeck, J. P. (2003). MrBayes 3: Bayesian phylogenetic inference under mixed models. Bioinformatics, 19:1572-1574.

Roos, C. (2003). Molekulare Phylogenie der Halbaffen, Schlankaffen und Gibbons. PhD thesis, Technische Universität München.

Roos, C., Schmitz, J., and Zischler, H. (2004). Primate jumping genes elucidate strepsirrhine phylogeny. Proceedings of the National Academy of Sciences, 101(29):10650-10654.

Rosenberg, N. A., Pritchard, J. K., Weber, J. L., Cann, H. M., Kidd, K. K., Zhivotovsky, L. A., and Feldman, M. W. (2002). Genetic structure of human populations. Science, 298(5602):2381-2385. 
Rozas, J., Sanchez-DelBarrio, J., Messeguer, X., and Rozas, R. (2003). DnaSP, DNA polymorphism analyses by the coalescent and other methods. Bioinformatics, 19(18):2496-2497.

Ryder, O. A. (1986). Species conservation and systematics: The dilemma of subspecies. Trends in Ecology and Evolution, 1(1):9-10.

Schmid, J. and Kappeler, P. (1994). Sympatric mouse lemurs (Microcebus, spp.) in western Madagascar. Folia Primatologica, 63:162-170.

Schradin, C. and Pillay, N. (2005). Intraspecific variation in the spatial and social organization of the African striped mouse. Journal of Mammalogy, 86(1):99-107.

Schülke, O., Kappeler, P., and Zischler, H. (2004). Small testes size despite high extra-pair paternity in the pair-living nocturnal primate Phaner furcifer. Behavioral Ecology and Sociobiology, 55:296-310.

Schulte-Hostedde, A. and Millar, J. (2004). Intraspecific variation of testis size and sperm length in the yellow-pine chipmunk (Tamias amoenus): implications for sperm competition and reproductive success. Behavioral Ecology and Sociobiology, 55(3):272-277.

Schwarz, E. (1931). A revision of the genera and species of Madagascar Lemuridae. Proceedings of the Zoological Society of London, pages 399-426.

Sechrest, W., Brooks, T., da Fonseca, G., Konstant, W., Mittermeier, R., Purvis, A., Rylands, A., and Gittleman, J. (2002). Hotspots and the conservation of evolutionary history. Proceedings of the National Academy of Sciences, 99(4):2067-2071.

Seiffert, E., Simons, E., and Attia, Y. (2003). Fossil evidence for an ancient divergence of lorises and galagos. Nature, 422:421-424.

Simons, E. L., Burney, D. A., Chatrath, P. S., Godfrey, L. R., Jungers, W. L., and Rakotosamimanana, B. (1995). AMS ${ }^{14} \mathrm{C}$ dates for extinct lemurs from caves in the Ankarana Massif, northern Madagascar. Quaternary Research, 43(2):249-254.

Smale, L., Nunes, S., and Holekamp, K. (1997). Sexually dimorphic dispersal in mammals: Patterns, causes, and consequences. Advances in the Study of Behavior, 26(181-250).

Smith, A., Horning, N., and Moore, D. (1997). Regional biodiversity planning and lemur conservation with GIS in western Madagascar. Conservation Biology, 11(2):498-512.

Sneath, P. and Sokal, R. (1973). Numerical Taxonomy. WH Freeman, San Francisco.

Sommer, S. (2001). Reproductive ecology of the endangered monogamous Malagasy giant jumping rat, Hypogeomys antimena. Mammalian Biolology, 66:111-115.

Sparks, J. S. and Smith, W. L. (2004). Phylogeny and biogeography of the Malagasy and Australasian rainbowfishes (Teleostei: Melanotaenioidei): Gondwanan vicariance and evolution in freshwater. Molecular Phylogenetics and Evolution, 33(3):719-734. 
Steenbeek, R., Sterck, E., de Vries, H., and van Hooff, J. (2000). Costs and benefits of the one-male, age-graded and all-male phases in wild Thomas's langur groups. In Kappeler, P., editor, Primate Males, pages 130-145. Cambridge University Press, Cambridge.

Storey, M., Mahoney, J., Saunders, A., Duncan, R., Kelley, S., and Coffin, M. (1995). Timing of hot spot-related volcanism and the breakup of Madagascar and India. Science, 267(5199):852-855.

Sugg, D. and Chesser, R. (1994). Effective population sizes with multiple paternity. Genetics, 137(4):1147-1155.

Surridge, A., Bell, D., Ibrahim, K., and Hewitt, G. (1999). Population structure and genetic variation of European wild rabbits (Oryctolagus cuniculus) in East Anglia. Heredity, 82 (Pt 5):479-487.

Swofford, D. (2002). PAUP* Phylogenetic analysis using parsimony (*and other methods). Vers. 4, Sinauer Associates, Sunderland, MA.

Tattersall, I. (1982). The Primates of Madagascar. Columbia University Press, New York.

Tattersall, I. (2007). Madagascar's lemurs: Cryptic diversity or taxonomic inflation? Evolutionary Anthropology, 16:12-23.

Tavare, S., Marshall, C. R., Will, O., Soligo, C., and Martin, R. D. (2002). Using the fossil record to estimate the age of the last common ancestor of extant primates. Nature, 416(6882):726-729.

Thalmann, U. (2000). Greater dwarf lemurs from the Bongolava (central western Madagascar). Lemur News, 5:33-35.

Thalmann, U. and Rakotoarison, N. (1994). Distribution of lemurs in central western Madagascar, with a regional distribution hypothesis. Folia Primatologica, 63:156-161.

Travis, S. E., Slobodchikoff, C. N., and Keim, P. (1995). Ecological and demographic effects on intraspecific variation in the social system of prairie dogs. Ecology, 76(6):1794-1803.

Trizio, I., Crestanello, B., Galbusera, P., Wauters, L. A., Tosi, G., Matthysen, E., and Hauffe, H. C. (2005). Geographical distance and physical barriers shape the genetic structure of Eurasian red squirrels (Sciurus vulgaris) in the Italian Alps. Molecular Ecolology, 14(2):469481.

van Schaik, C. and Kappeler, P. (2003). The evolution of social monogamy in primates. In Reichard, U. and Boesch, C., editors, Monogamy: Mating Strategies and Partnerships in Birds, Humans and other Mammals, pages 59-80. Cambridge University Press, Cambridge.

Vences, M. (2004). Origin of Madagascar's extant fauna: A perspective from amphibians, reptiles and other non-flying vertebrates. Italian Journal of Zoology, Suppl. 2:217-228. 
Vences, M., Freyhof, J., Sonnenberg, R., Kosuch, J., and Veith, M. (2001). Reconciling fossils and molecules: Cenozoic divergence of cichlid fishes and the biogeography of Madagascar. Journal of Biogeography, 28:1091-1099.

Vences, M., Kosuch, J., Rodel, M.-O., Lotters, S., Channing, A., Glaw, F., and Bohme, W. (2004). Phylogeography of Ptychadena mascareniensis suggests transoceanic dispersal in a widespread African-Malagasy frog lineage. Journal of Biogeography, 31(4):593-601.

Warren, B. H., Bermingham, E., Bowie, R. C. K., Prys-Jones, R. P., and Thebaud, C. (2003). Molecular phylogeography reveals island colonization history and diversification of western Indian Ocean sunbirds (Nectarinia: Nectariniidae). Molecular Phylogenetics and Evolution, 29(1):67-85.

Watterson, G. (1984). Lines of descent and the coalescent. Theoretical Population Biology, 26(1):77-92.

Weber, M. (1928). Die Säugetiere. Band 2: Systematischer Teil. Gustav Fischer Verlag, Jena, 2nd edition.

Weir, B. and Cockerham, C. (1984). Estimating F-statistics for the analysis of population structure. Evolution, 38:1358-1370.

Wells, N. (2003). Some hypotheses on the Mesozoic and Cenozoic paleoenvironmental history of Madagascar. In Goodman, S. and Benstead, J., editors, The Natural History of Madagascar, pages 16-74. The University of Chicago Press, Chicago.

Wells, N. A. and Andriamihaja, B. (1993). The initiation and growth of gullies in Madagascar: are humans to blame? Geomorphology, 8(1):1-46.

Wilmé, L., Goodman, S., and Ganzhorn, J. (2006). Biogeographic evolution of Madagascar's microendemic biota. Science, 312(5776):1063-1065.

Wimmer, B., Tautz, D., and Kappeler, P. (2002). The genetic population structure of the gray mouse lemur (Microcebus murinus), a basal primate from Madagascar. Behavioral Ecology and Sociobiology, 52(2):166-175.

Wise, C., Sraml, M., Rubinsztein, D., and Easteal, S. (1997). Comparative nuclear and mitochondrial genome diversity in humans and chimpanzees. Molecular Biology and Evolution, 14(7):707-716.

Wright, P. and Martin, L. (1995). Predation, pollination and torpor in two nocturnal prosimians: Cheirogaleus major and Microcebus rufus in the rain forest of Madagascar. In Alterman, L., Doyle, G., and Izard, M., editors, Creatures of the Dark: The Nocturnal Prosimians, chapter 4. Plenum Press.

Yamagiwa, J. and Hill, D. (1998). Intraspecific variation in the social organization of Japanese macaques: Past and present scope of field studies in natural habitats. Primates, 39:257-273. 
Yang, Z. and Yoder, A. D. (2003). Comparison of likelihood and bayesian methods for estimating divergence times using multiple gene loci and calibration points, with application to a radiation of cute-looking mouse lemur species. Systematic Biology, 52(5):705-716.

Yoder, A. (1997). Back to the future: A synthesis of strepsirrhine systematics. Evolutionary Anthropology, 6(1):11-22.

Yoder, A., Cartmill, M., Ruvolo, M., Smith, K., and Vilgalys, R. (1996). Ancient single origin for Malagasy primates. Proceedings of the National Academy of Sciences, 93:5122-5126.

Yoder, A., Olson, L., Hanley, C., Heckman, K., Rasoloarison, R., Russell, A., Ranivo, J., Soarimalala, V., Karanth, K., Raselimanana, A., and Goodman, S. (2005). A multidimensional approach for detecting species patterns in Malagasy vertebrates. Proceedings of the National Academy of Sciences, USA, 102 (Suppl 1):6587-6594.

Yoder, A., Rasoloarison, R., Goodman, S., Irwin, J., Atsalis, S., Ravosa, M., and Ganzhorn, J. (2000). Remarkable species diversity in Malagasy mouse lemurs (Primates, Microcebus). Proceedings of the National Academy of Sciences, 97(21):11325-11330.

Yoder, A. and Yang, Z. (2004). Divergence dates for Malagasy lemurs estimated from multiple gene loci: Geological and evolutionary context. Molecular Ecology, 13(4):757-773.

Yoder, A. D. (2007). Lemurs. Current Biology, 17(20):R866-R868.

Yoder, A. D., Burns, M. M., Zehr, S., Delefosse, T., Veron, G., Goodman, S. M., and Flynn, J. J. (2003). Single origin of Malagasy carnivora from an African ancestor. Nature, 421(6924):734-737.

Yoder, A. D. and Nowak, M. D. (2006). Has vicariance or dispersal been the predominant biogeographic force in Madagascar? Only time will tell. Annual Review of Ecology, Evolution, and Systematics, 37(1):405-431.

Ziegler, T. and Snowdon, C. (2000). Preparental hormone levels and parenting experience in male cotton-top tamarins, Saguinus oedipus. Hormones and Behavior, 38(3):159-167.

Zimmermann, E., Cepok, S., Rakotoarison, N., Zietemann, V., and Radespiel, U. (1998). Sympatric mouse lemurs in north-west Madagascar: A new rufous mouse lemur species (Microcebus ravelobensis). Folia Primatologica, 69:106-114.

Zink, R. (1996). Bird species diversity. Nature, 381(6583):566.

Zwickl, D. J. (2006). Genetic Algorithm Approaches for the Phylogenetic Analysis of Large Biological Sequence Datasets under the Maximum Likelihood Criterion. PhD thesis, The University of Texas at Austin. 
A Appendix:

Species delimitation - Morphometrics 


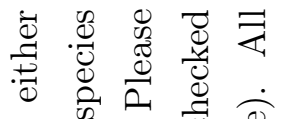

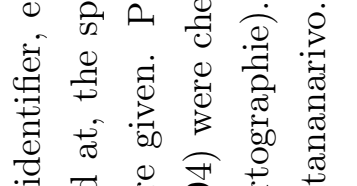

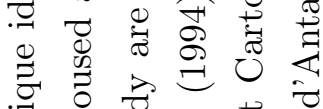

.

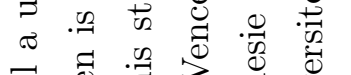

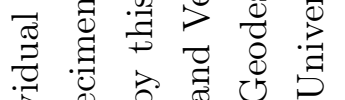

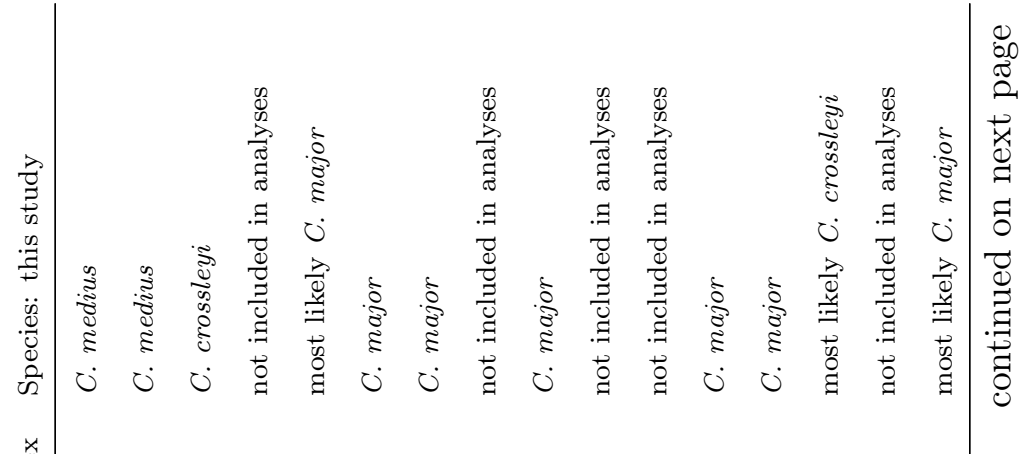

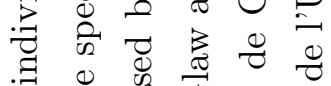

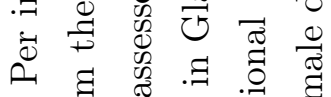

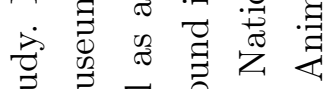

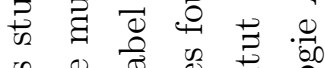

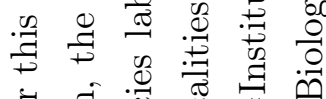

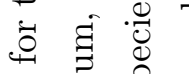

उ

苛

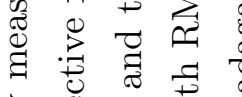

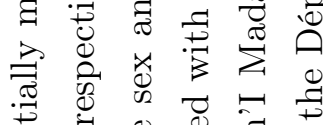

.尹.尹

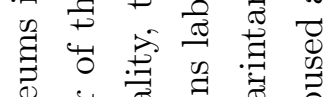

范

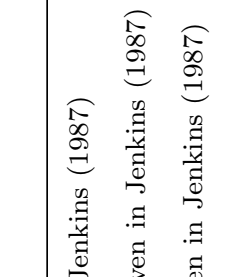

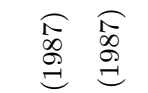

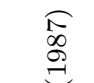

当

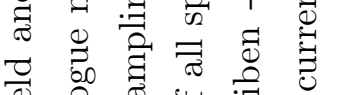

业

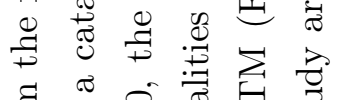

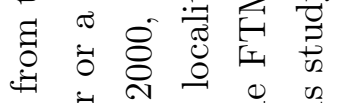

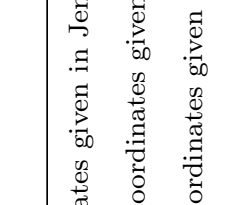

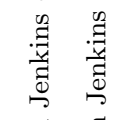

$\exists . \Xi \quad \frac{9}{\square}$

击

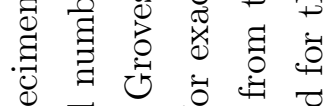

की

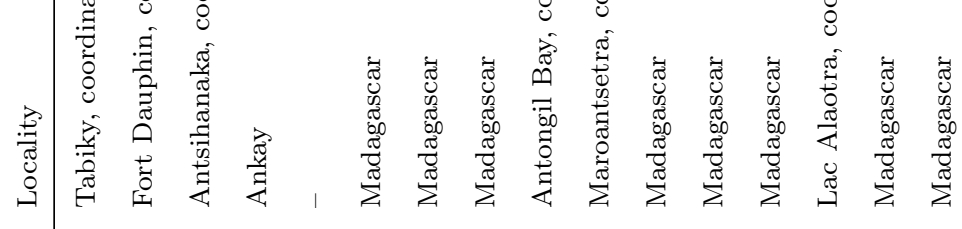

节

๘

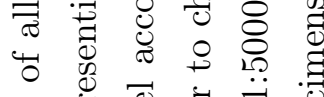

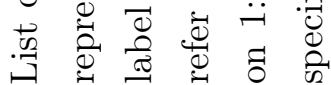

$\ddot{\leftrightarrow}$

$\frac{\sqrt{6}}{\frac{\pi}{4}}$

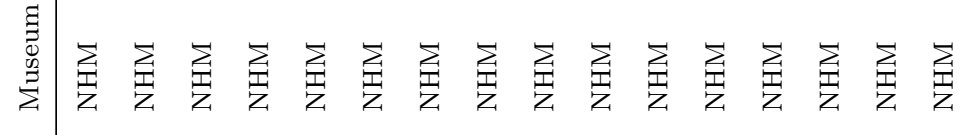

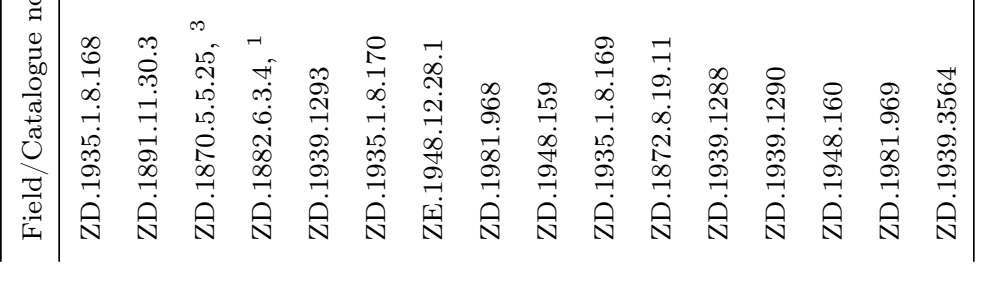




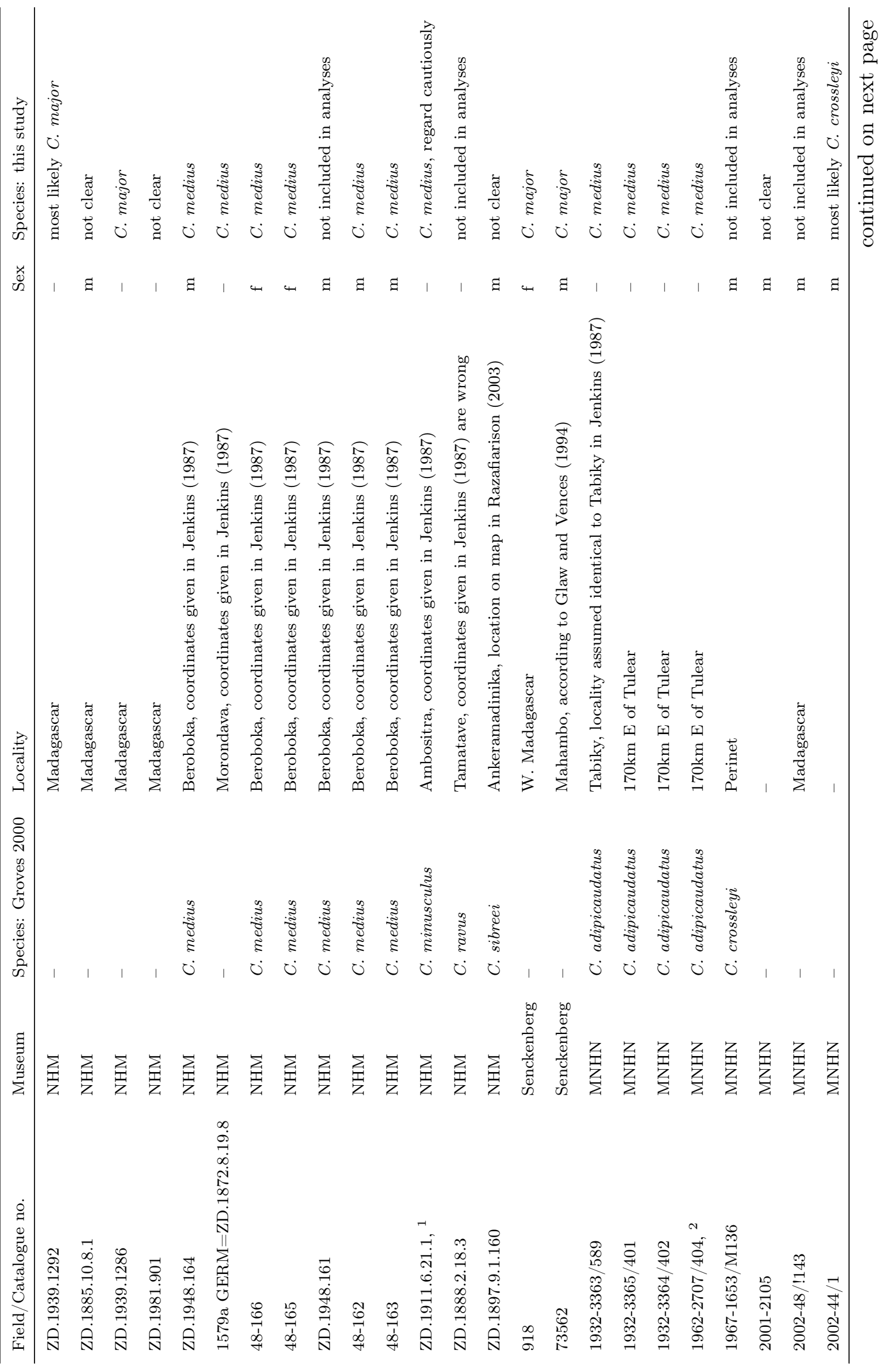




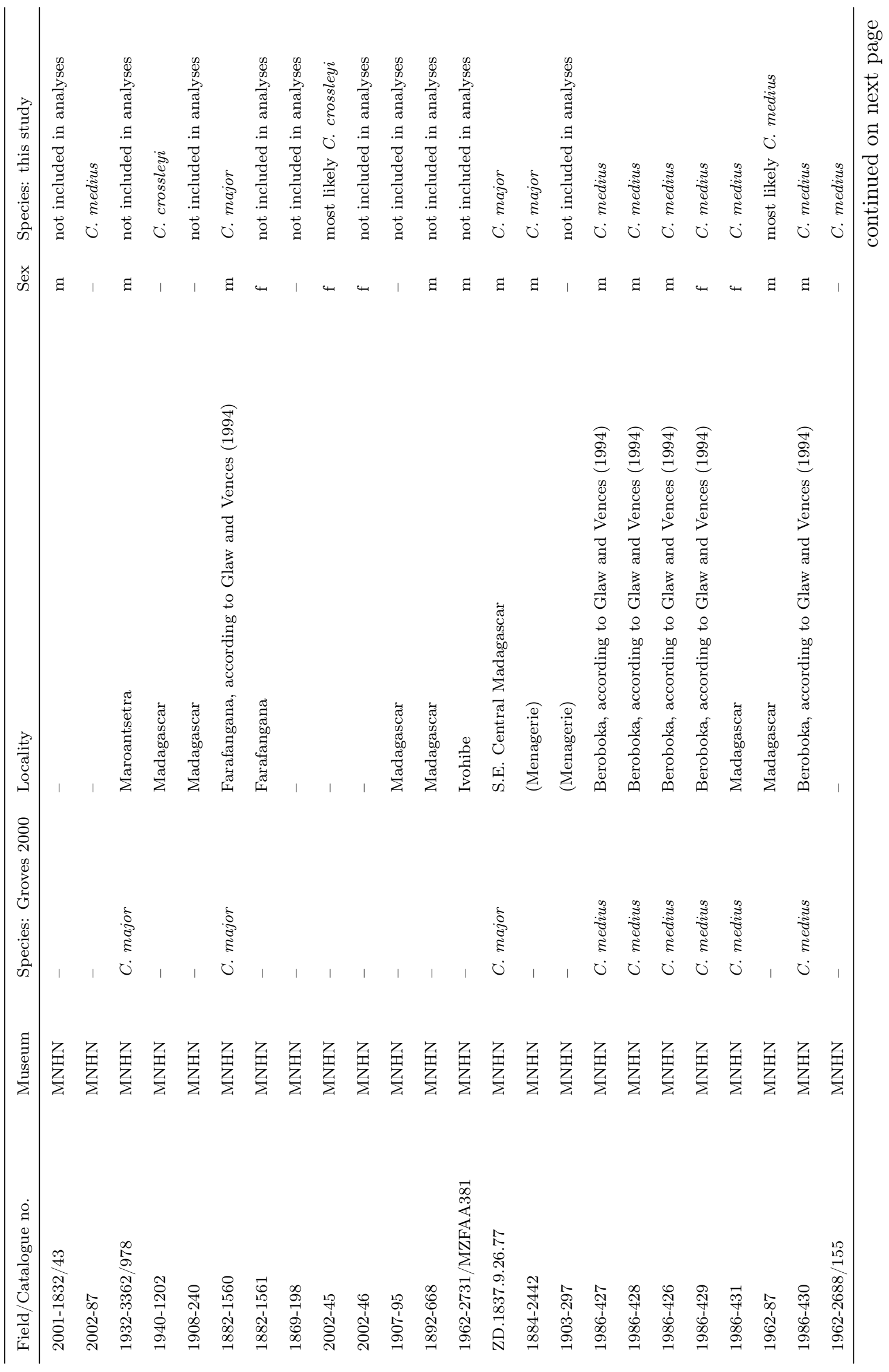




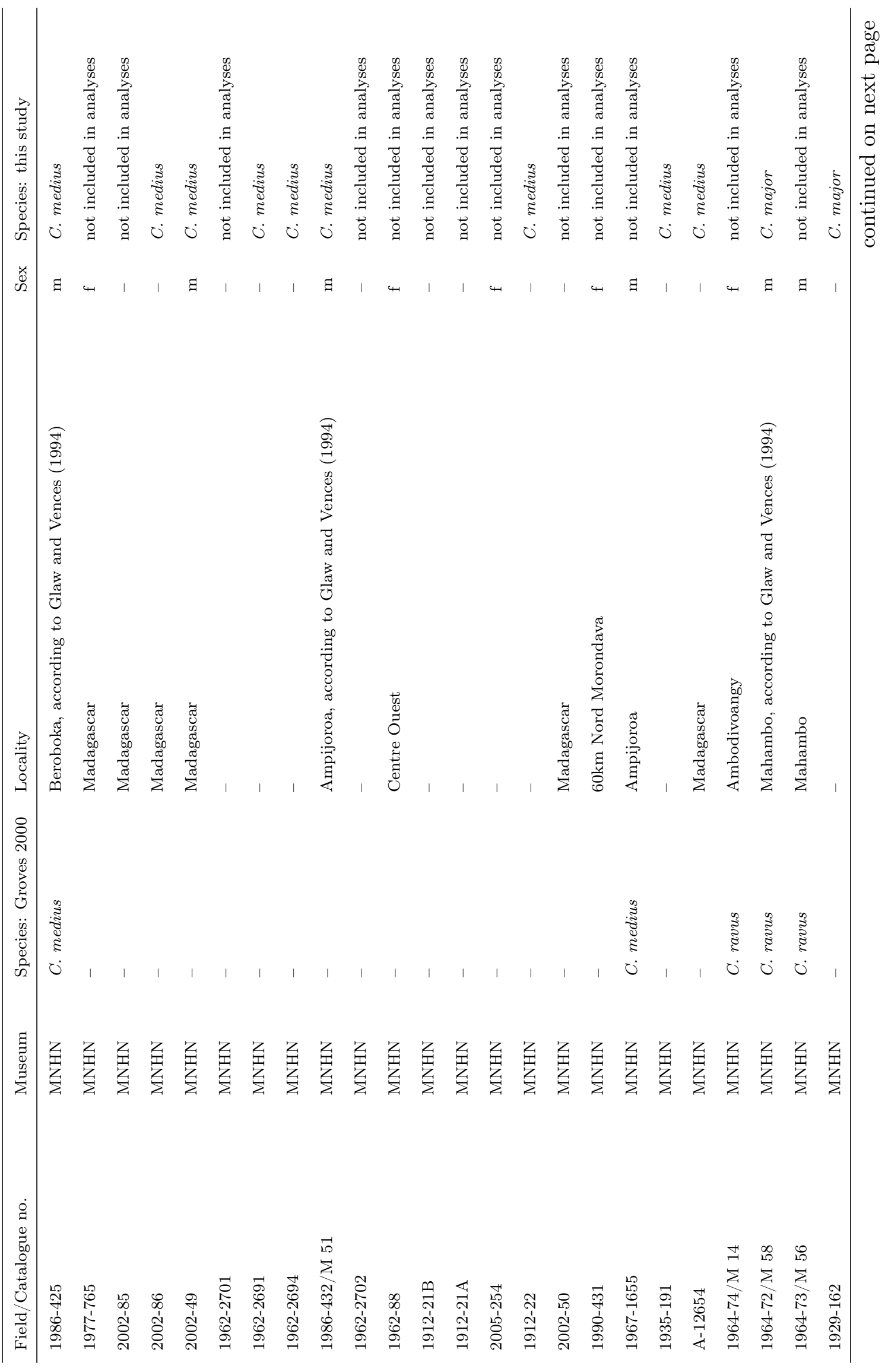




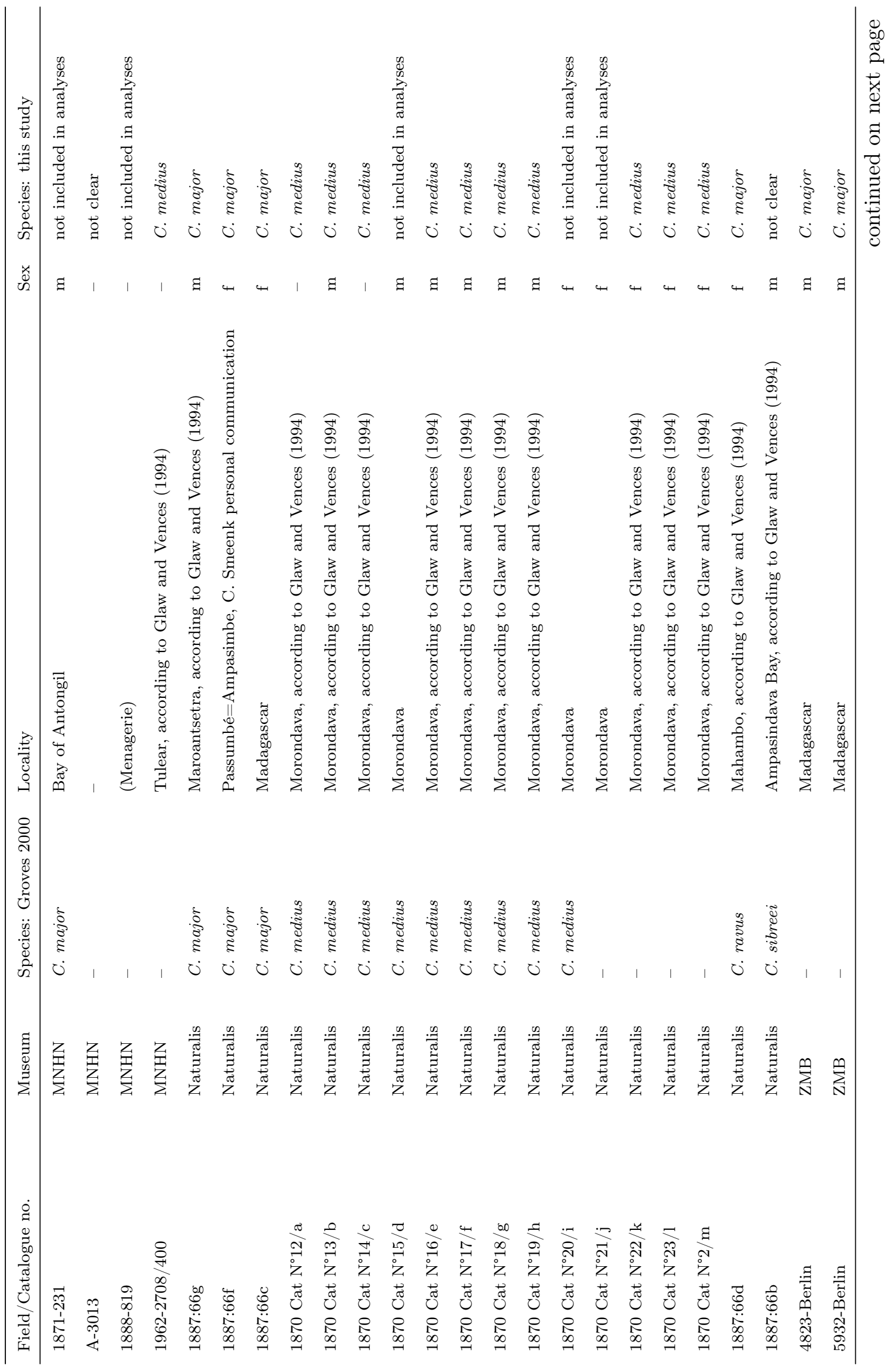




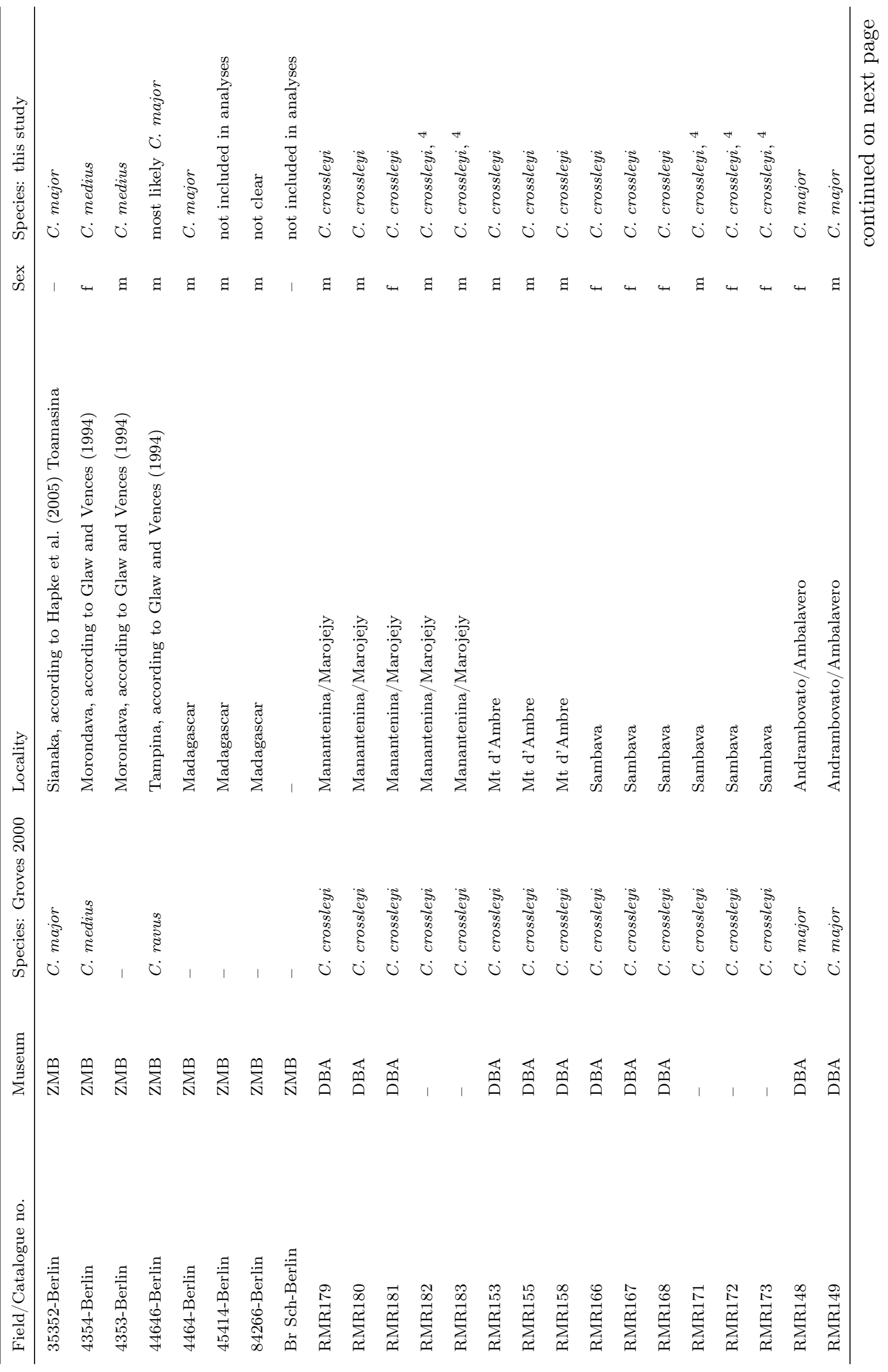




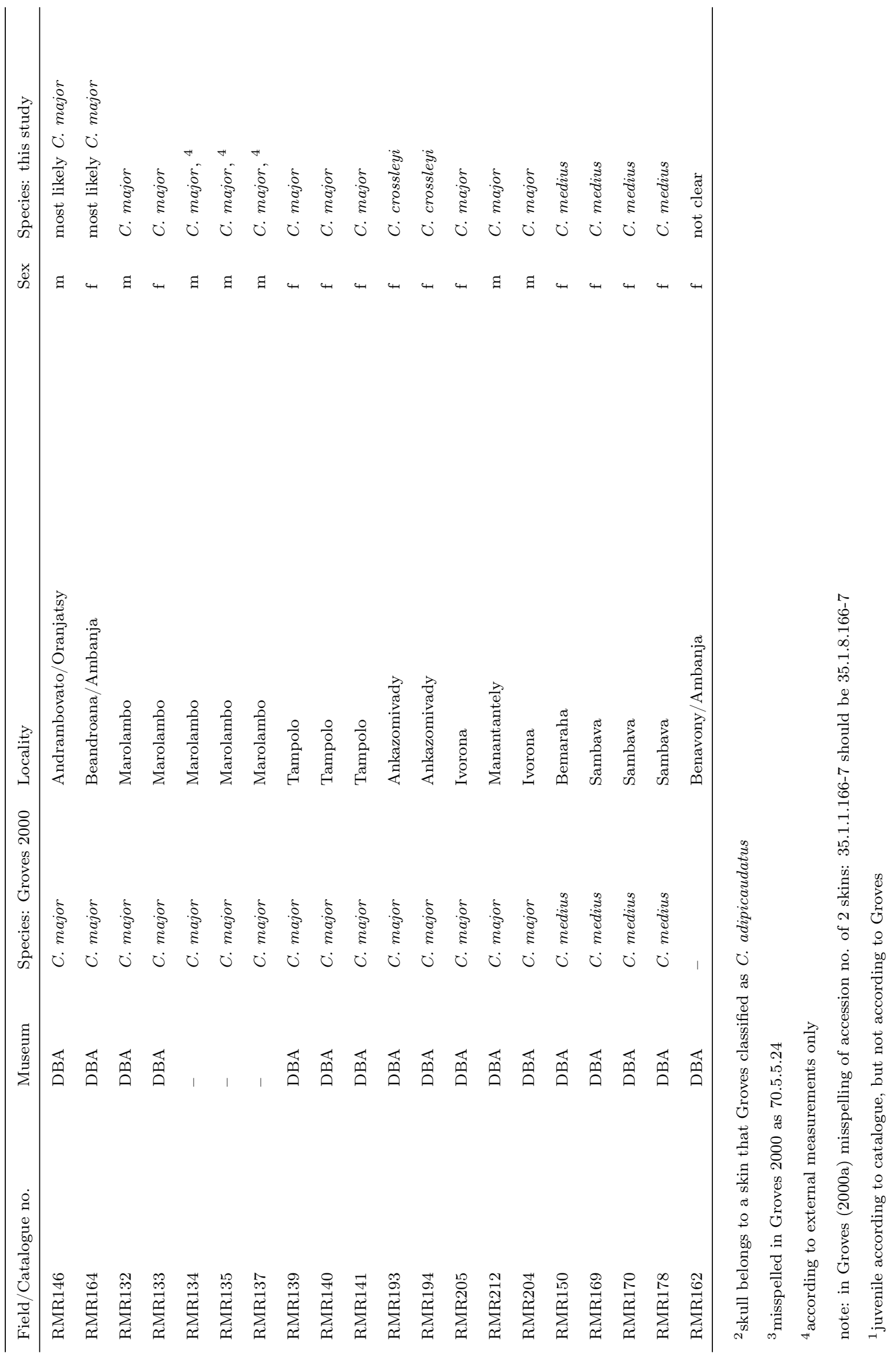




\section{B Appendix:}

Species delimitation - Genetics 


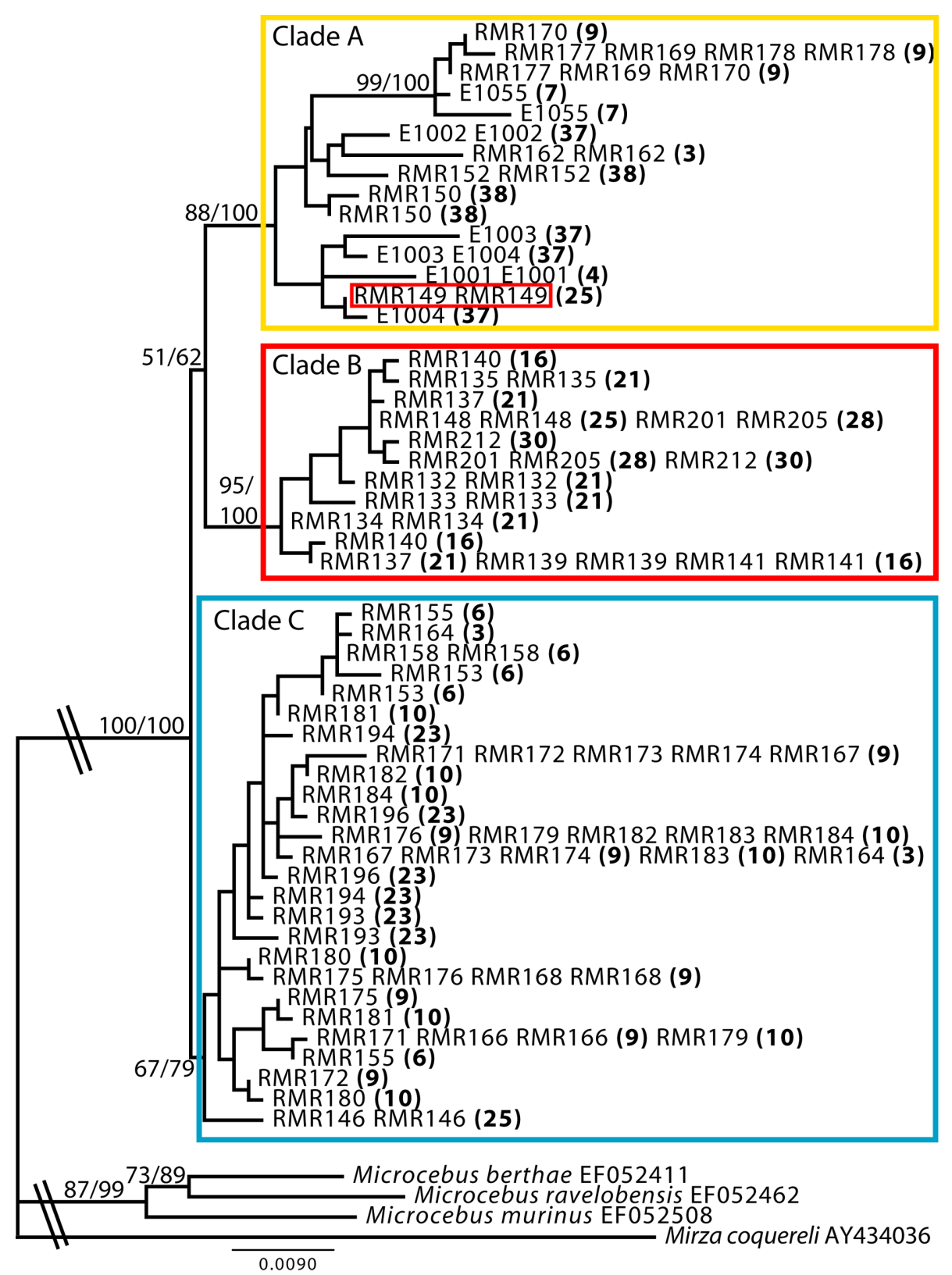

Figure B.1: Maximum likelihood phylogram based on an alignment of $v W F$ haplotype sequences from 48 field samples. Tip labels contain the individual field numbers (E, RMR) of sequences within a haplotype. The sampling locality a haplotype was found in, are given in bold type in parentheses. Maximum likelihood bootstrap values and Bayesian posterior probabilities are depicted above the branches. Groups of haplotypes found in individuals that correspond to clades A, B and C in the mtDNA tree are outlined by the colored frames. 


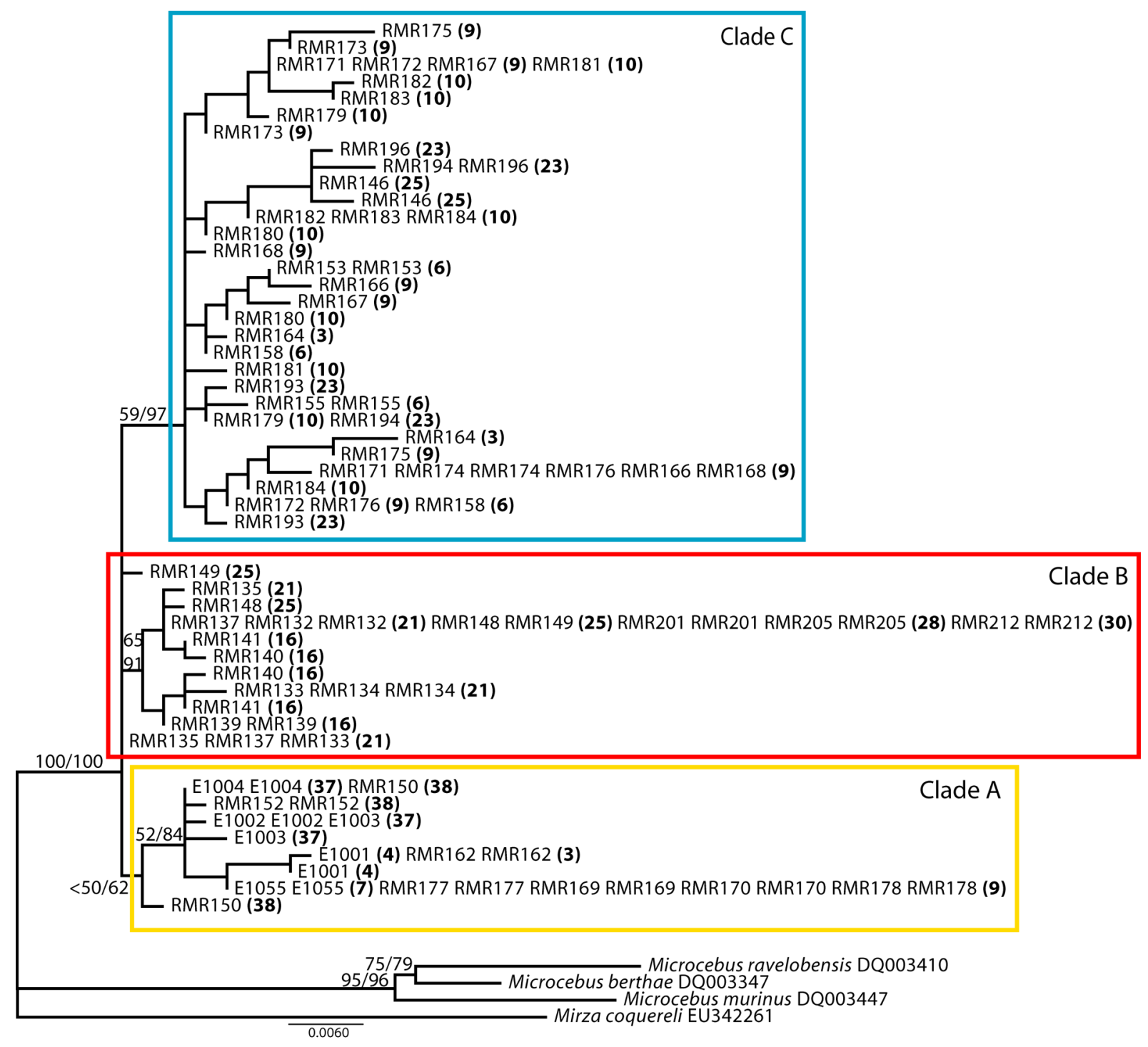

Figure B.2: Maximum likelihood phylogram based on an alignment of $f i b a$ haplotype sequences from 48 field samples. Tip labels contain the individual field numbers (E, RMR) of sequences within a haplotype. The sampling locality a haplotype was found in, are given in bold type in parentheses. Maximum likelihood bootstrap values and Bayesian posterior probabilities are depicted above the branches. Groups of haplotypes found in individuals that correspond to clades $\mathrm{A}, \mathrm{B}$ and $\mathrm{C}$ in the mtDNA tree are outlined by the colored frames. 


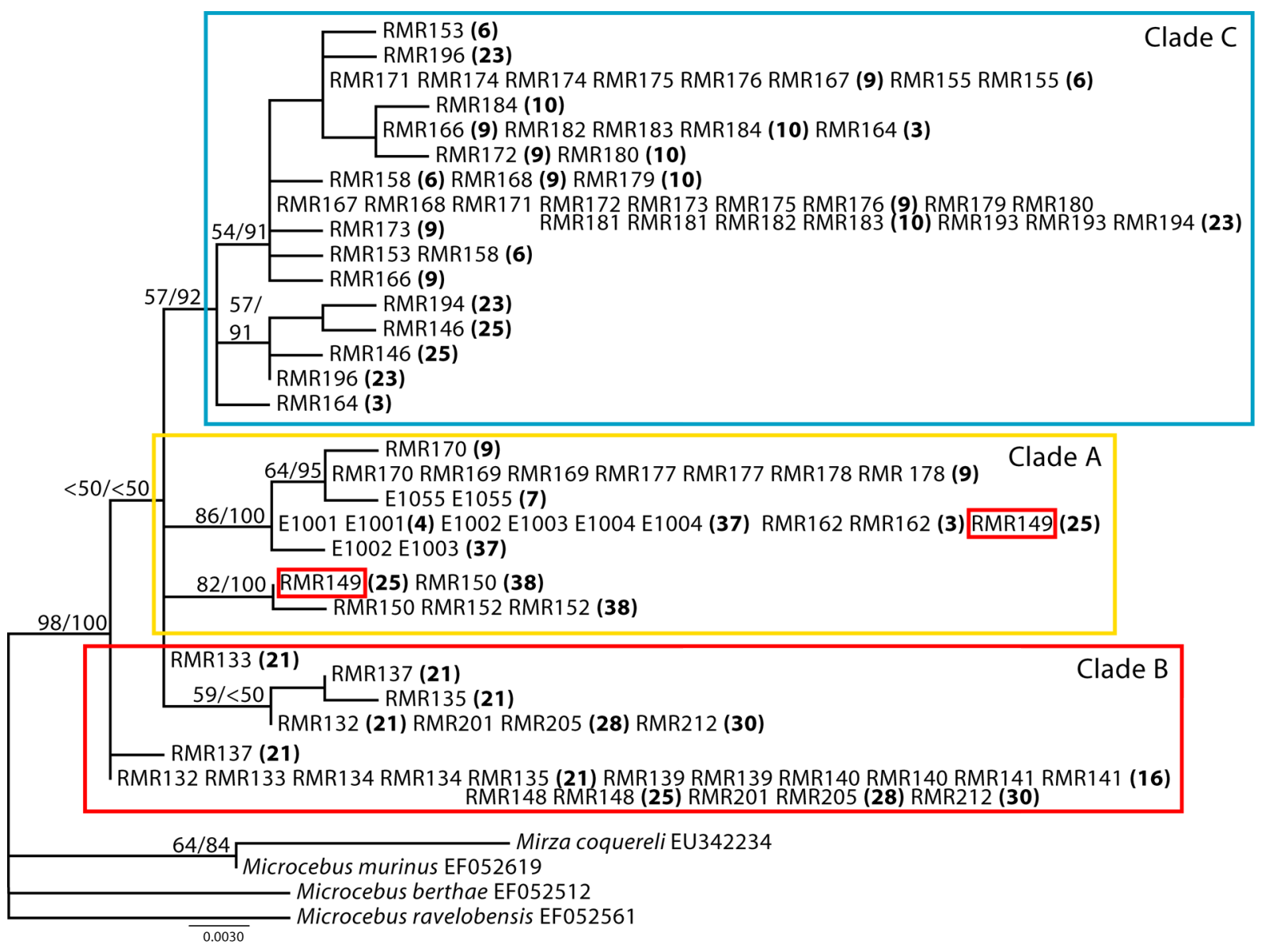

Figure B.3: Maximum likelihood phylogram based on an alignment of adora3 haplotype sequences from 48 field samples. Tip labels contain the individual field numbers (E, RMR) of sequences within a haplotype. The sampling locality a haplotype was found in, are given in bold type in parentheses. Maximum likelihood bootstrap values and Bayesian posterior probabilities are depicted above the branches. Groups of haplotypes found in individuals that correspond to clades A, B and C in the mtDNA tree are outlined by the colored frames. 
A)

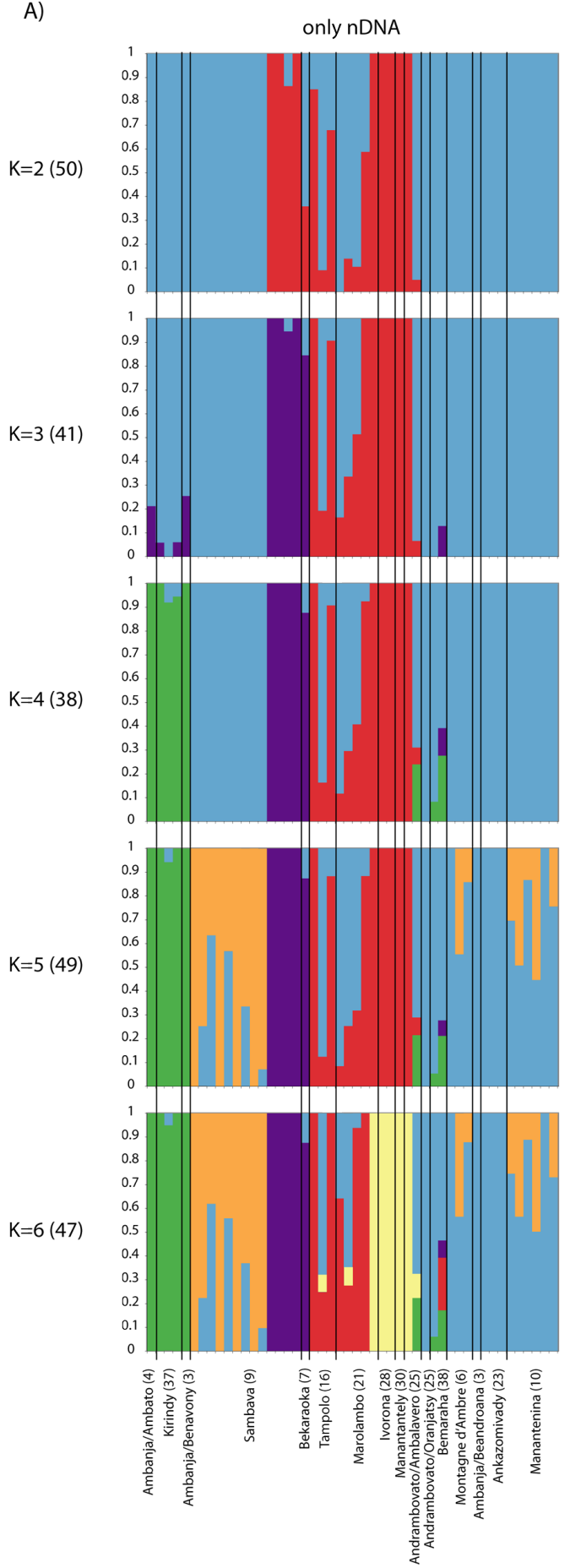

B)

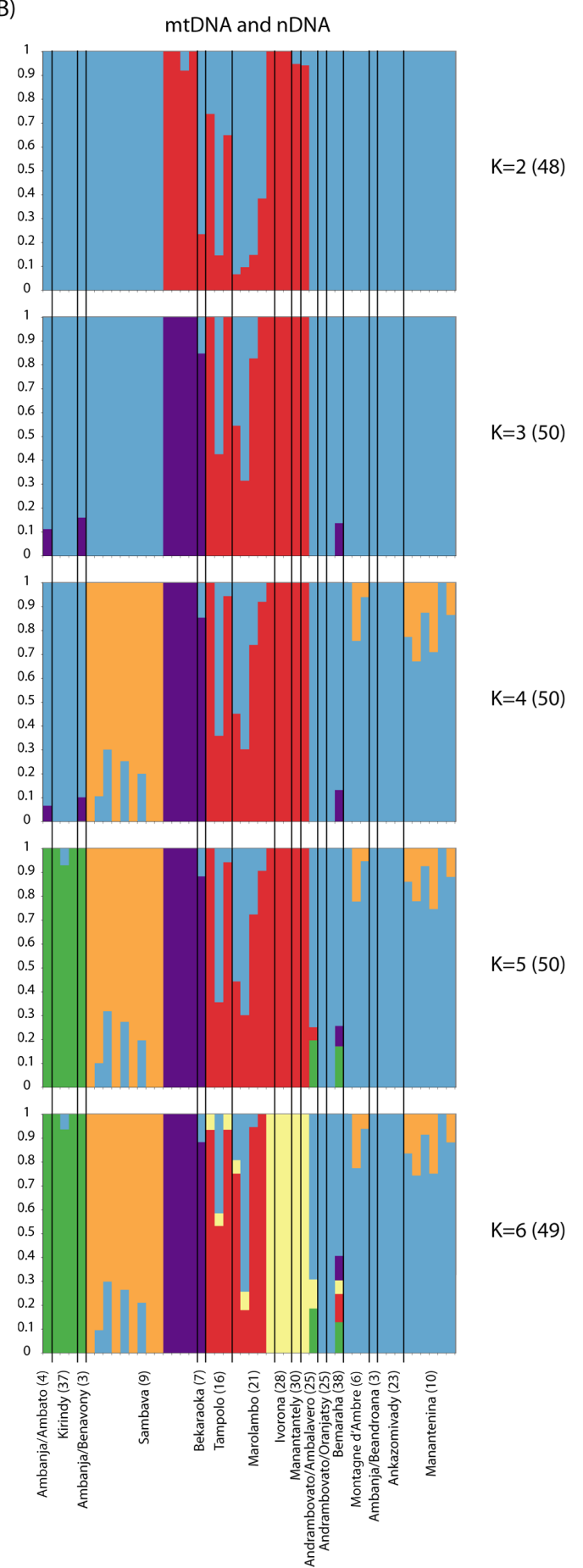

Figure B.4: Bayesian assignment of the 48 field-collected individuals to populations assuming a population number of $\mathrm{K}=2$ to $\mathrm{K}=6$. Individuals are arrayed along the $\mathrm{x}$ axis. The $\mathrm{y}$ axis denotes the cumulative posterior probability of an individuals placement in particular population(s). Numbers in parentheses for each $\mathrm{K}$ indicate the number of identical solutions at a $95 \%$ threshold. Individuals are divided into populations by thin black lines. Populations are labeled at the bottom with numbers in parentheses corresponding to the sampling locality as marked in Fig. 3.1. (A) Results based on nuclear loci. (B) Results based on all loci. The solutions for each $K$, from $K=2$ to $K=6$, are consistent in that at each $K+1$ the assignment of individuals to the clusters remains stable with just one cluster being split into two. There is only one exception to this pattern. The individual from Bekaraoka switches clusters from $\mathrm{K}=2$ to $\mathrm{K}=3$. 
C Appendix:

Phylogeography 


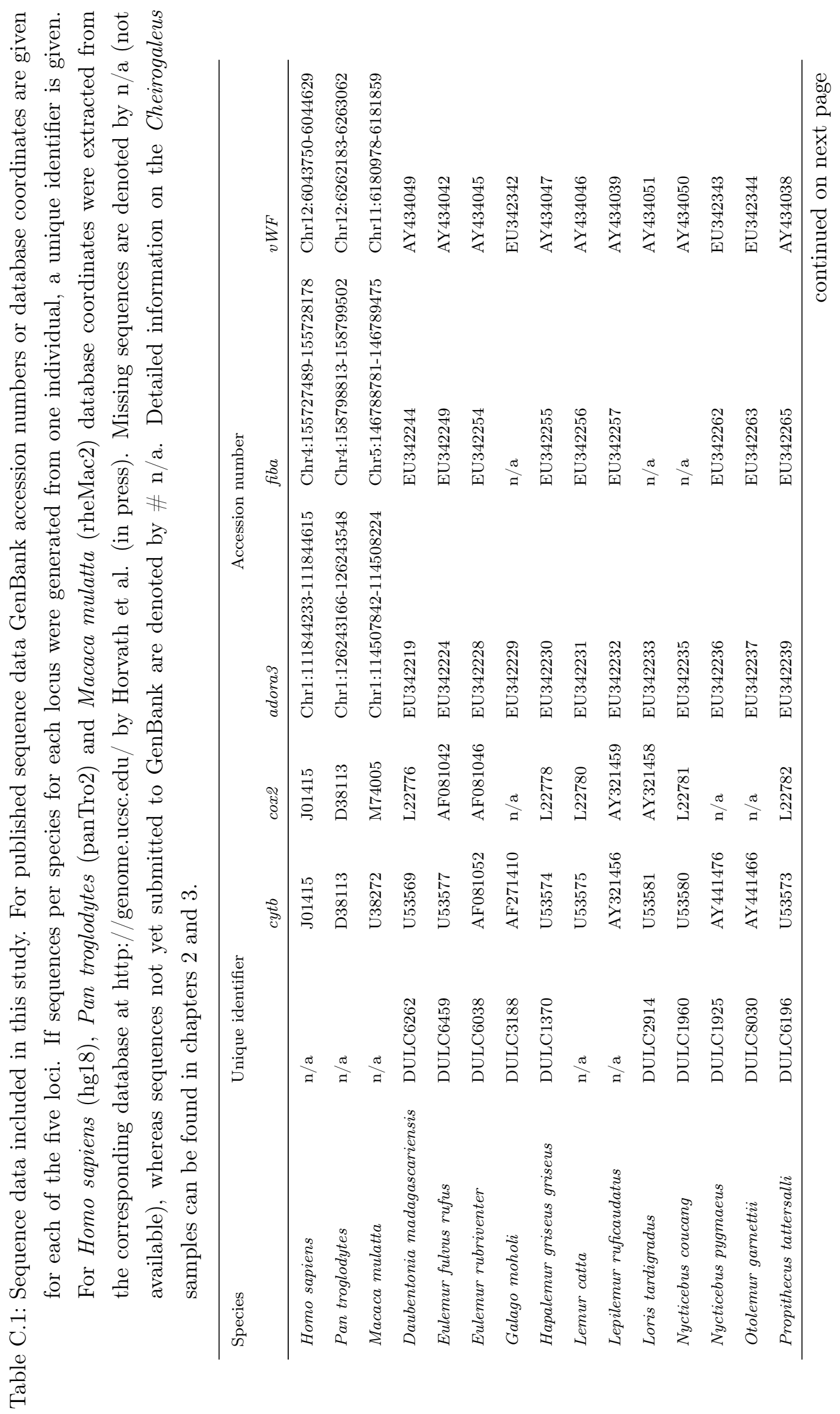




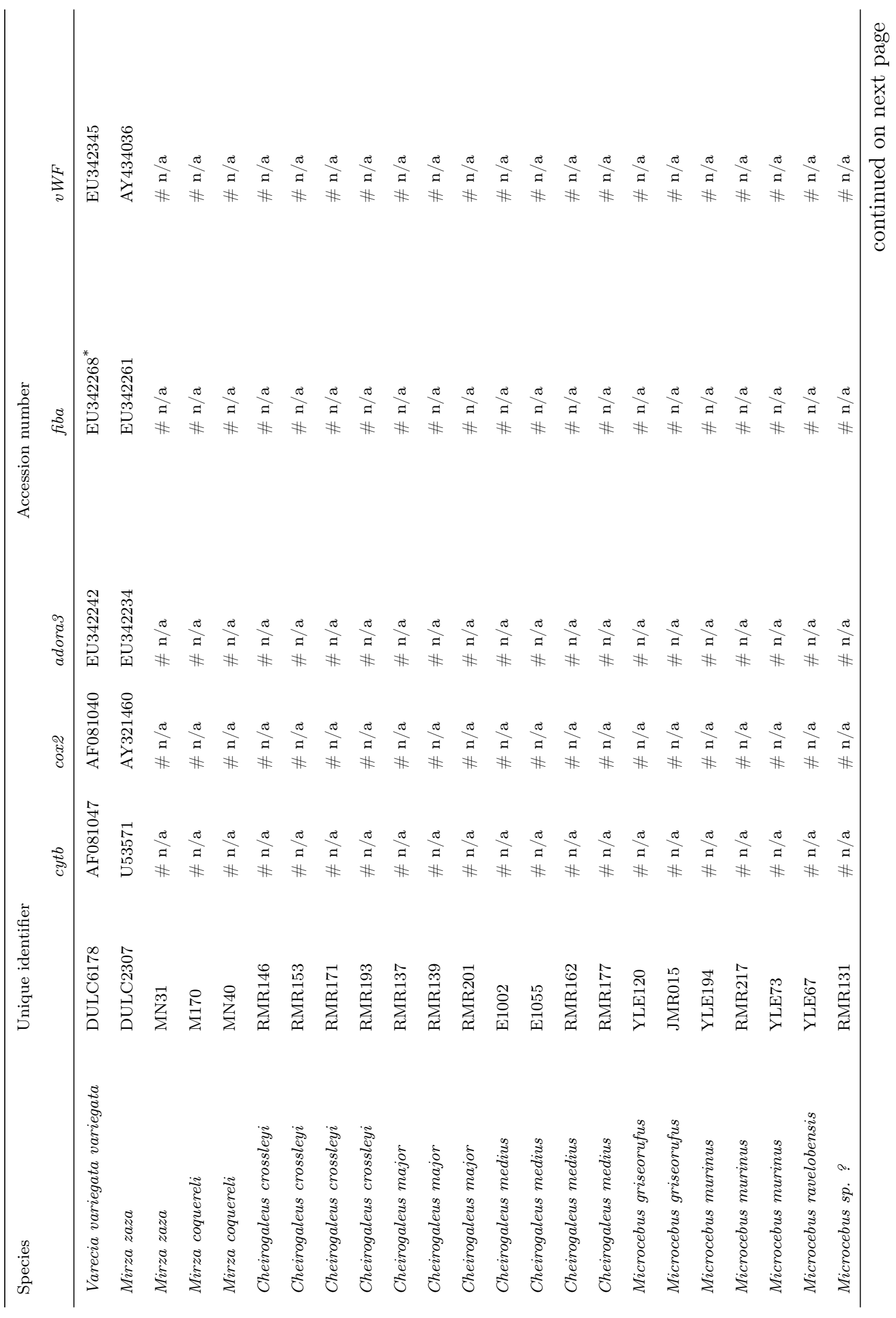




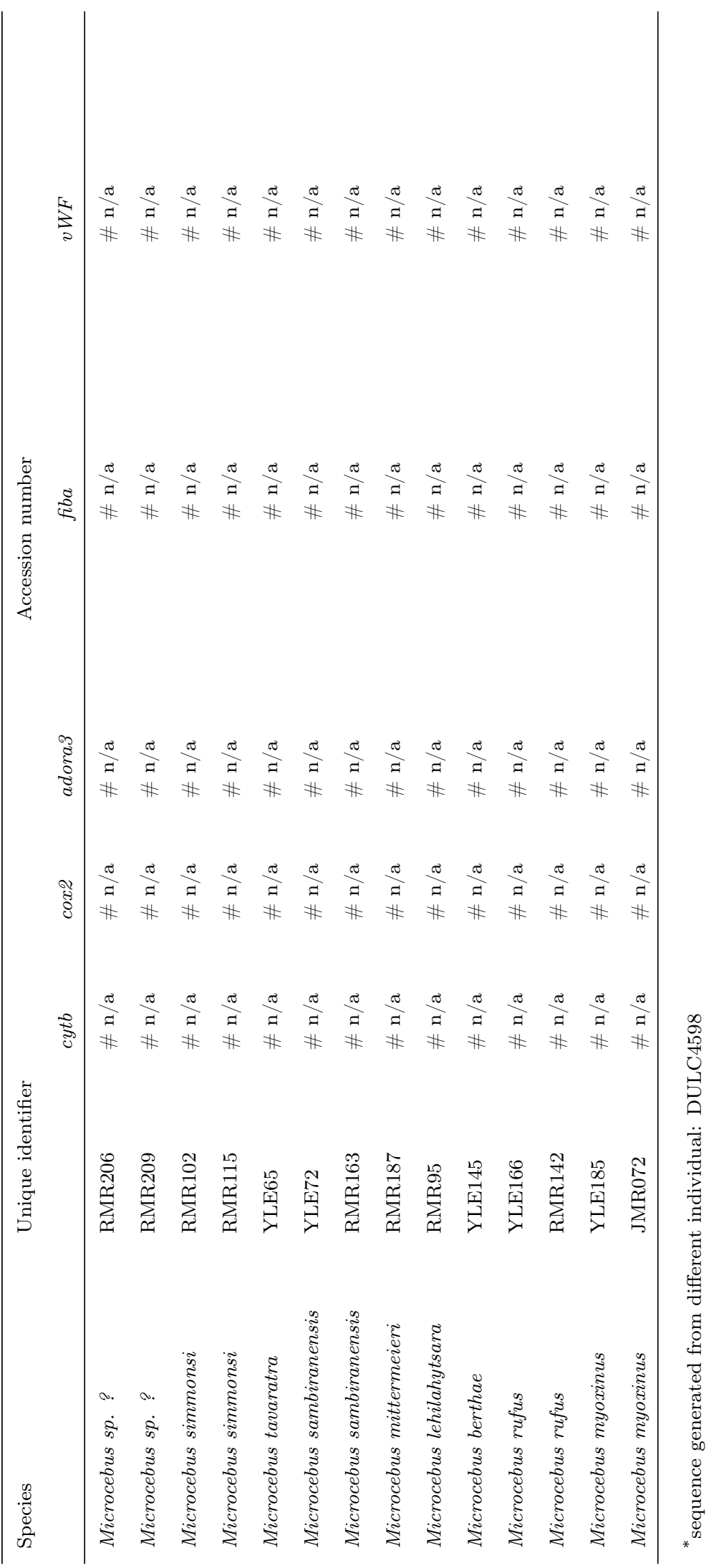




\section{Acknowledgements}

I thank my "Doktorvater" Peter M. Kappeler for giving me the opportunity to work on this topic, for introducing me to the wonderful and mysterious fauna of Madagascar, and for always having had an open ear for my worries and ideas.

Many thanks go to my colleague Rodin Rasoloarison for putting up with the non-meateating yellow girl with the dirty hands in the field.

I am extremely thankful to Christina Oberdieck, who not only helped me in the lab, but also made sure that it was fun! My other great help in the lab was the Hachinger; thanks for all the solutions to stupid pipetting, primer ordering or cloning problems. Keep the Lederhosen on!

I thank Dave Weisrock for the endless skype-discussions about the BEAUti and the BEAST, trees and creative English writing. I sincerely thank Anne Yoder for inviting me to Duke University, where I got to experience a very stimulating scientific environment. I am especially grateful to my "Doktoropa" Peter Klopfer and his wife Martha Klopfer, who invited me to stay with them during my visit in 2006 at Duke University. The many discussions over a good glass of wine, whether on biological topics or others, were inspiring and surely have made a lasting impression on me.

I thank Dietmar Zinner for his help with GIS maps and keeping me busy with baboons, when dwarf lemurs were scarce.

Many thanks go to my colleagues in the Department of Sociobiology and Behavioral Ecology at the German Primate Center and the Department of Sociobiology and Anthropology at the Georg-August-University of Göttingen; without you it would have been only half the fun! Special thanks in this regard go to my office mate Melanie Dammhahn, whose personal library also saved me from so many annoying trips to the SUB and to Vanessa Mass. I will miss Wednesday evenings!

For their support at all conceivable levels, be it regular shipments of homemade jam or relentless computer support, especially in form of clusters waiting to be busied with my endless MCMC runs, I want to thank my parents.

Mountains of thanks to all my flatmates who made sure that, in the end, I did like Göttingen: Katrin Lux, Maike Böcker, Yvonne Melcher, Peter Köpper, Moritz von Dungern, Arne Bischoff, Oli Eberhard. I will sincerely miss Lotze 13 with all of its contents!

I want to express my deep gratitude to Moritz Rahlfs, who helped me immensely during the last stage of this $\mathrm{PhD}$ and made sure that I stayed sane - thanks for putting up with my tears! Alles wird gut. 


\section{Erklärung über eigene Leistungen}

Ich versichere, dass ich die vorliegende Arbeit selbstständig verfasst und keine anderen als die angegebenen Hilfsmittel verwendet habe. Die Stellen, die anderen Werken wörtlich oder sinngemäß entnommen sind, sind als solche kenntlich gemacht. Eigene Beiträge im Verhältnis zu denen von Koautoren bei bereits publizierten oder zur Publikation einzureichenden Teilen dieser Arbeit sind wie folgt:

Kapitel 2: Die Feldarbeiten wurden hauptsächlich von Rodin Rasoloarison, unter zeitweiliger Mitwirkung von Linn F. Groeneveld, durchgeführt. Ebenso hat Rodin Rasoloarison alle morphometrischen Messungen der Feld- und Museumsexemplare vorgenommen. Die Datenauswertung, sowie das Verfassen des Manuskripts wurde von Linn F. Groeneveld durchgeführt.

Kapitel 3: Die Feldarbeiten wurden hauptsächlich von Rodin Rasoloarison, unter zeitweiliger Mitwirkung von Linn F. Groeneveld, durchgeführt. Die Entnahme von Proben an $\mathrm{Mu}-$ seumsexemplaren, die Laborarbeiten, genetischen Analysen und die Datenauswertung wurden von Linn F. Groeneveld durchgeführt. Das Manuskript wurde, in Rücksprache mit Dave Weisrock, von Linn F. Groeneveld verfasst.

Kapitel 4: Die Ausgangsdaten für dieses Kapitel ergeben sich aus den Arbeiten aus Kapitel 2 und 3. Zusätzliche Sequenzdaten der Gattung Microcebus wurden von Dave Weisrock bereitgestellt. Alle Datenauswertungen wurden von Linn F. Groeneveld ausgeführt. Das Manuskript wurde von Linn F. Groeneveld verfasst.

Kapitel 5: Die Feldarbeiten wurden hauptsächlich von Linn F. Groeneveld durchgeführt. Die Laborarbeiten, genetischen Analysen und die Datenauswertung wurden von Tina Fredsted durchgeführt. Das Manuskript wurde von Tina Fredsted verfasst.

Ich versichere weiterhin, dass diese Arbeit in gleicher oder ähnlicher Form noch keiner anderen Prüfungsbehörde vorgelegen hat. 